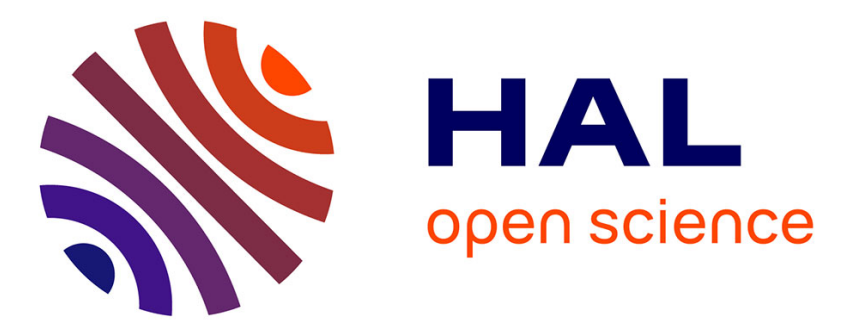

\title{
On the motion law of fronts for scalar reaction-diffusion equations with equal depth multiple-well potentials : the degenerate case.
}

Fabrice Bethuel, Didier Smets

\section{- To cite this version:}

Fabrice Bethuel, Didier Smets. On the motion law of fronts for scalar reaction-diffusion equations with equal depth multiple-well potentials: the degenerate case.. Chinese Annals of Mathematics Series B, 2018, 38 (1), pp.83-148. 10.1007/s11401-016-1064-3 . hal-01481388

\section{HAL Id: hal-01481388 \\ https://hal.sorbonne-universite.fr/hal-01481388}

Submitted on 15 Dec 2021

HAL is a multi-disciplinary open access archive for the deposit and dissemination of scientific research documents, whether they are published or not. The documents may come from teaching and research institutions in France or abroad, or from public or private research centers.
L'archive ouverte pluridisciplinaire HAL, est destinée au dépôt et à la diffusion de documents scientifiques de niveau recherche, publiés ou non, émanant des établissements d'enseignement et de recherche français ou étrangers, des laboratoires publics ou privés. 


\title{
On the motion law of fronts for scalar reaction-diffusion equations with equal depth multiple-well potentials: the degenerate case
}

\author{
Fabrice BETHUEL* and Didier SMETS ${ }^{\dagger}$
}

\begin{abstract}
We derive a precise motion law for fronts of solutions to scalar one-dimensional reaction-diffusion equations with multiple-wells, in the case the second derivative of the potential vanishes at its minimizers. We show that, renormalizing time in an algebraic way, the motion of fronts is governed by a simple system of ordinary differential equations of nearest neighbor interaction type. These interactions may be either attractive or repulsive. Our results are not constrained by the possible occurence of collisions nor splittings. They present substantial differences with the results obtained in the case the second derivative does not vanish at the wells, a case which has been extensively studied in the literature, and where fronts have been showed to move at exponentially small speed, with motion laws which are not renormalizable.
\end{abstract}

\section{Introduction}

This paper is a continuation of our previous works $[4,5]$ where we analyzed the behavior of solutions $v$ of the reaction-diffusion equation of gradient type

$(\mathrm{PGL})_{\varepsilon}$

$$
\frac{\partial v_{\varepsilon}}{\partial t}-\frac{\partial^{2} v_{\varepsilon}}{\partial x^{2}}=-\frac{1}{\varepsilon^{2}} \nabla V\left(v_{\varepsilon}\right)
$$

where $0<\varepsilon<1$ is a small parameter. In [5], we considered the case where the potential $V$ is a smooth map from $\mathbb{R}$ to $\mathbb{R}^{k}$ with multiple wells whose second derivative vanishes at the wells. The main result there, stated in Theorem 1 here, provides an upper bound for the speed of fronts. In the present paper we restrict ourselves to the scalar case, $k=1$, and provide a precise motion law for the fronts, showing in particular that the upper bound provided in [5] is sharp. We assume throughout this paper that the potential $V$ is a smooth function from $\mathbb{R}$ to $\mathbb{R}$ which satisfies the following assumptions:

$\left(\mathrm{A}_{1}\right) \quad \inf V=0$ and the set of minimizers $\Sigma \equiv\{y \in \mathbb{R}, V(y)=0\}$ is finite,

with at least two distinct elements, that is

$$
\Sigma=\left\{\sigma_{1}, \ldots, \sigma_{\mathfrak{q}}\right\}, \mathfrak{q} \geq 2, \sigma_{1}<\cdots<\sigma_{\mathfrak{q}} .
$$

*UPMC-Paris6, UMR 7598 LJLL, Paris, F-75005 France,

${ }^{\dagger}$ UPMC-Paris6, UMR 7598 LJLL, Paris, F-75005 France 
$\left(\mathrm{A}_{2}\right)$ There exists a number $\theta>1$ such that for all $i$ in $\{1, \cdots, \mathfrak{q}\}$, we have

$$
V(u)=\lambda_{i}\left(u-\sigma_{i}\right)^{2 \theta}+\underset{u \rightarrow \sigma_{i}}{o}\left(\left(u-\sigma_{i}\right)^{2 \theta}\right), \text { where } \lambda_{i}>0 .
$$

$\left(\mathrm{A}_{3}\right)$ There exists constants $\alpha_{\infty}>0$ and $R_{\infty}>0$ such that

$$
u \cdot \nabla V(u) \geq \alpha_{\infty}|u|^{2}, \text { if }|u|>R_{\infty} .
$$

Whereas assumption $\left(A_{1}\right)$ expresses the fact that the potential possesses at least two minimizers, also termed wells, and $\left(\mathrm{A}_{3}\right)$ describes the behavior at infinity, and is of a more technical nature, assumption $\left(\mathrm{A}_{2}\right)$, which is central in the present paper, describes the local behavior near the minimizing wells. The number $\theta$ is of course related to the order of vanishing of the derivatives near zero. Since $\theta>1$, then $V^{\prime \prime}\left(\sigma_{i}\right)=0$, and $\left(\mathrm{A}_{2}\right)$ holds if and only if

$$
\frac{d^{j}}{d u^{j}} V\left(\sigma_{i}\right)=0 \text { for } j=1, \cdots, 2 \theta-1 \text { and } \frac{d^{2 \theta}}{d u^{2 \theta}} V\left(\sigma_{i}\right)>0,
$$

with

$$
\lambda_{i}=\frac{1}{(2 \theta) !} \frac{d^{2 \theta}}{d u^{2 \theta}} V\left(\sigma_{i}\right) .
$$

A typical example of such potentials is given by $V(u)=\left(1-u^{2}\right)^{2 \theta}=(1-u)^{2 \theta}(1+u)^{2 \theta}$ which has two minimizers, +1 and -1 , so that $\Sigma=\{+1,-1\}$, minimizers vanishing at order $2 \theta$. In this paper, the order of degeneracy is an integer assumed to be the same at all wells: fractional or site dependent orders may presumably be handled with the same tools, however at the cost of more complicated statements.

We recall that equation $(\mathrm{PGL})_{\varepsilon}$ corresponds to the $L^{2}$ gradient-flow of the energy functional $\mathcal{E}$ which is defined for a function $u: \mathbb{R} \mapsto \mathbb{R}$ by the formula

$$
\mathcal{E}_{\varepsilon}(u)=\int_{\mathbb{R}} e_{\varepsilon}(u)=\int_{\mathbb{R}} \frac{\varepsilon|\dot{u}|^{2}}{2}+\frac{V(u)}{\varepsilon} .
$$

As in $[4,5]$, we consider only finite energy solutions. More precisely, we fix an arbitrary constant $M_{0}>0$ and we consider the condition

$$
\mathcal{E}_{\varepsilon}(u) \leq M_{0}<+\infty .
$$

Besides the assumptions on the potential, the main assumption is on the initial data $v_{\varepsilon}^{0}(\cdot)=$ $v_{\varepsilon}(\cdot, 0)$, assumed to satisfy $\left(\mathrm{H}_{0}\right)$ independently of $\varepsilon$. In particular, in view of the classical energy identity

$$
\mathcal{E}_{\varepsilon}\left(v_{\varepsilon}\left(\cdot, T_{2}\right)\right)+\varepsilon \int_{T_{1}}^{T_{2}} \int_{\mathbb{R}}\left|\frac{\partial v_{\varepsilon}}{\partial t}\right|^{2}(x, t) d x d t=\mathcal{E}_{\varepsilon}\left(v_{\varepsilon}\left(\cdot, T_{1}\right)\right) \quad \forall 0 \leq T_{1} \leq T_{2},
$$

we have

$$
\mathcal{E}_{\varepsilon}\left(v_{\varepsilon}(\cdot, t)\right) \leq M_{0}, \forall t \geq 0 .
$$

This implies in particular that for every given $t \geq 0$, we have $V\left(v_{\varepsilon}(x, t)\right) \rightarrow 0$ as $|x| \rightarrow \infty$. It is then quite straightforward to deduce from assumption $\left(\mathrm{H}_{0}\right),\left(\mathrm{A}_{1}\right),\left(\mathrm{A}_{2}\right)$ as well as the energy identity (1), that $v_{\varepsilon}(x, t) \rightarrow \sigma_{ \pm}$as $x \rightarrow \pm \infty$, where $\sigma_{ \pm} \in \Sigma$ do not depend on $t$. In other words, for any time, our solutions connect to given minimizers of the potential. 


\subsection{Main results: Fronts and their speed}

The notion of fronts is central in the dynamics. For a map $u: \mathbb{R} \mapsto \mathbb{R}$, the set

$$
\mathcal{D}(u) \equiv\left\{x \in \mathbb{R}, \operatorname{dist}(u(x), \Sigma) \geq \mu_{0}\right\},
$$

is termed throughout the front set of $u$. The constant $\mu_{0}$ which appears in its definition is fixed once for all, sufficiently small so that

$$
\frac{\lambda_{i}}{2}\left(u-\sigma_{i}\right)^{2 \theta} \leq V(u) \leq \frac{1}{\theta} V^{\prime}(u)\left(u-\sigma_{i}\right) \leq 4 V(u) \leq 8 \lambda_{i}\left(u-\sigma_{i}\right)^{2 \theta},
$$

for each $i \in\{1, \cdots, \mathfrak{q}\}$ and whenever $\left|u-\sigma_{i}\right| \leq \mu_{0}$. The front set corresponds to the set of points where $u$ is "far" from the minimizers $\sigma_{i}$, and hence where transitions from one minimizer to the other may occur. A straightforward analysis yields

Lemma 1 (see e.g. [4]). Assume that $u$ verifies $\left(H_{0}\right)$. Then there exists $\ell$ points $x_{1}, \ldots, x_{\ell}$ in $\mathcal{D}(u)$ such that

$$
\mathcal{D}(u) \subset \cup_{k=1}^{\ell}\left[x_{k}-\varepsilon, x_{k}+\varepsilon\right],
$$

with a bound $\ell \leq \frac{M_{0}}{\eta_{0}}$ on the number of points, $\eta_{0}$ being some constant depending only on $V$.

In view of Lemma 1 , the measure of the front sets is of order $\varepsilon$, and corresponds to a small neighborhood of order $\varepsilon$ of the points $x_{i}$. Notice that if $\left(u_{\varepsilon}\right)_{\varepsilon>0}$ is a family of functions satisfying $\left(\mathrm{H}_{0}\right)$ then it is well-known that the family is locally bounded in $B V(\mathbb{R}, \mathbb{R})$ and hence locally compact in $L^{1}(\mathbb{R}, \mathbb{R})$. Passing to a subsequence if necessary, we may assert that

$$
u_{\varepsilon} \rightarrow u^{\star} \text { in } L_{\mathrm{loc}}^{1}(\mathbb{R})
$$

where $u^{\star}$ takes values in $\Sigma$ and is a step function. More precisely there exist an integer $\ell \leq \frac{M_{0}}{\eta_{0}}, \ell$ points $a_{1}<\cdots<a_{\ell}$ and a function $\hat{\imath}:\left\{\frac{1}{2}, \cdots, \frac{1}{2}+\ell\right\} \rightarrow\{1, \cdots, \mathfrak{q}\}$ such that

$$
u^{\star}=\sigma_{\hat{\imath}\left(k+\frac{1}{2}\right)} \text { on }\left(a_{k}, a_{k+1}\right) \text {, }
$$

for $k=0, \cdots, \ell$, and where we use the convention $a_{0}:=-\infty$ and $a_{\ell+1}:=+\infty$. The points $a_{k}$, for $k=1 \cdots, \ell$, are the limits as $\varepsilon$ shrinks to 0 of the points $x_{i}$ provided by Lemma 1 (the number and the positions of which are of course $\varepsilon$ dependent), so that the front set $\mathcal{D}\left(u_{\varepsilon}\right)$ shrinks as $\varepsilon$ tends to 0 to a finite set. In the sequel, we shall refer to step functions with values into $\Sigma$ as steep front chains and we will write

$$
u^{\star}=u^{\star}\left(\ell, \hat{\imath},\left\{a_{k}\right\}\right)
$$

to determine them unambigously.

We go back to equation $(\mathrm{PGL})_{\varepsilon}$ and consider a family of functions $\left(v_{\varepsilon}\right)_{\varepsilon>0}$ defined on $\mathbb{R} \times \mathbb{R}^{+}$ which are solutions to the equation $(\mathrm{PGL})_{\varepsilon}$ and satisfy the energy bound $\left(\mathrm{H}_{0}\right)$. We set

$$
\mathcal{D}_{\varepsilon}(t)=\mathcal{D}\left(v_{\varepsilon}(\cdot, t)\right) .
$$

The evolution of the front set $\mathcal{D}_{\varepsilon}(t)$ when $\varepsilon$ tends to 0 is the main focus of our paper. The following result ${ }^{1}$ has been proved in [5]:

\footnotetext{
${ }^{1}$ which holds also more generally for systems.
} 
Theorem $1([5])$. There exists constants $\rho_{0}>0$ and $\alpha_{0}>0$, depending only on the potential $V$ and on $M_{0}$ such that if $r \geq \alpha_{0} \varepsilon$, then

$$
\mathcal{D}_{\varepsilon}(t+\Delta t) \subset \mathcal{D}_{\varepsilon}(t)+[-r, r], \quad \text { for every } t \geq 0,
$$

provided $0 \leq \Delta t \leq \rho_{0} r^{2}\left(\frac{r}{\varepsilon}\right)^{\frac{\theta+1}{\theta-1}}$.

As a matter of fact, it follows from this result that the average speed of the front set at that length-scale should not exceed

$$
c_{\mathrm{ave}} \simeq \frac{r}{(\Delta t)_{\max }} \leq \rho_{0}^{-1} r^{-(\omega+1)} \varepsilon^{\omega},
$$

where

$$
\omega=\frac{\theta+1}{\theta-1} .
$$

Notice that $1<\omega<+\infty$ and that the upper bound provided by (4) decreases with $\theta$, that is, the more degenerate the minimizers of $V$ are, the higher the possible speed allowed by the bound (4). In contrast, the speed is at most exponentially small in the case of non degenerate potentials (see e.g. [9], [4] and the references therein). One aim of the present paper is to show that the upper bound provided by the estimate (4) is in fact optimal ${ }^{2}$ and actually to derive a precise motion law for the fronts. An important fact, on which our results are built, is the following observation ${ }^{3}$ :

$$
\text { Equation }(P G L)_{\varepsilon} \text { is renormalizable. }
$$

This assertion means that, rescaling time in an appropriate way, the evolution of fronts in the asymptotic limit $\varepsilon \rightarrow 0$ is governed by an ordinary differential equation which does not involve the parameter $\varepsilon$. More precisely, we accelerate time by the factor $\varepsilon^{-\omega}$ and consider the new time $s=\varepsilon^{\omega} t$. In the accelerated time, we consider the map

$$
\mathfrak{v}_{\varepsilon}(x, s)=v_{\varepsilon}\left(x, s \varepsilon^{-\omega}\right) \text {, and set } \mathfrak{D}_{\varepsilon}(s)=\mathcal{D}\left(\mathfrak{v}_{\varepsilon}(\cdot, s)\right) .
$$

It follows from Theorem 1 that for given $r \geq \alpha_{0} \varepsilon$,

$$
\mathfrak{D}_{\varepsilon}(s+\Delta s) \subset \mathfrak{D}_{\varepsilon}(s)+[-r, r], \quad \text { for every } s \geq 0,
$$

provided that $0 \leq \Delta s \leq \rho_{0} r^{\omega+2}$.

Concerning the initial data, we will assume that there exists a steep front chain $v^{\star}\left(\ell_{0}, \hat{\imath}_{0},\left\{a_{k}^{0}\right\}\right)$ such that

$$
\left\{\begin{aligned}
v_{\varepsilon}^{0} & \longrightarrow v^{\star}\left(\ell_{0}, \hat{\imath}_{0},\left\{a_{k}^{0}\right\}\right) \text { in } L_{\text {loc }}^{1}(\mathbb{R}), \\
\mathfrak{D}_{\varepsilon}(0) & \longrightarrow\left\{a_{k}^{0}\right\}_{1 \leq k \leq \ell_{0}}, \text { locally in the sense of the Hausdorff distance },
\end{aligned}\right.
$$

as $\varepsilon \rightarrow 0$. Let us emphasize that assumption $\left(\mathrm{H}_{1}\right)$ is not restrictive, since it follows assuming only the energy bound $\left(\mathrm{H}_{0}\right)$ and passing possibly to a subsequence (see above). In our first result, we will impose the additional condition

$$
\left(\mathrm{H}_{\text {min }}\right) \quad\left|\hat{\imath}_{0}\left(k+\frac{1}{2}\right)-\hat{\imath}_{0}\left(k-\frac{1}{2}\right)\right|=1 \text { for } 1 \leq k \leq \ell_{0} .
$$

\footnotetext{
${ }^{2}$ at least in the scalar case considered here.

${ }^{3}$ which to our knowledge has not been observed before, even using formal arguments.
} 
This assumption could be rephrased as a "multiplicity one" condition: it means that the jumps consist of exactly one transition between consecutive minimizers $\sigma_{i}$ and $\sigma_{i \pm 1}$. To each transition point $a_{k}^{0}$ we may assign a sign, denoted by $\dagger_{k} \in\{+,-\}$, in the following way:

$$
\dagger_{k}=+ \text { if } \sigma_{\hat{\imath}_{0}\left(k+\frac{1}{2}\right)}=\sigma_{\hat{\imath}_{0}\left(k-\frac{1}{2}\right)}+1 \text { and } \dagger_{k}=- \text { if } \sigma_{\hat{\imath}_{0}\left(k+\frac{1}{2}\right)}=\sigma_{\hat{\imath}_{0}\left(k-\frac{1}{2}\right)}-1 .
$$

We consider next the system of ordinary differential equations

$$
\mathfrak{S}_{k} \frac{d}{d s} a_{k}=\frac{\Gamma_{k}^{+}}{\left(a_{k}-a_{k+1}\right)^{\omega+1}}-\frac{\Gamma_{k}^{-}}{\left(a_{k}-a_{k-1}\right)^{\omega+1}},
$$

for $1 \leq k \leq \ell_{0}$, where $\mathfrak{S}_{k}$ stands for the energy of the corresponding stationary front, namely

$$
\mathfrak{S}_{k}=\int_{\sigma_{\hat{\imath}_{0}\left(k-\frac{1}{2}\right)}}^{\sigma_{\hat{\imath}_{0}\left(k+\frac{1}{2}\right)}} \sqrt{2 V(u)} d u
$$

and where we have set, for $k=1, \cdots, \ell_{0}$

$$
\left\{\begin{array}{l}
\Gamma_{k}^{+}=2^{\omega}\left(\lambda_{\hat{\imath}_{0}\left(k+\frac{1}{2}\right)}\right)^{-\frac{1}{\theta-1}} \mathcal{A}_{\theta} \text { if } \dagger_{k}=-\dagger_{k+1} \\
\Gamma_{k}^{-}=-2^{\omega}\left(\lambda_{\hat{\imath}_{0}\left(k+\frac{1}{2}\right)}\right)^{-\frac{1}{\theta-1}} \mathcal{B}_{\theta} \text { if } \dagger_{k}=\dagger_{k+1} .
\end{array}\right.
$$

In (9), $\lambda_{\hat{\imath}_{0}\left(k+\frac{1}{2}\right)}$ is defined in $\left(\mathrm{A}_{2}\right)$ and the constants $\mathcal{A}_{\theta}>0$ and $\mathcal{B}_{\theta}>0$, depending only on $\theta$, are defined in (A.9) of Appendix A. Note in particular that $(\mathcal{S})$ is fully determined by the pair $\left(\ell_{0}, \hat{\imath}_{0}\right)$, and we shall therefore sometimes refer to it as $\mathcal{S}_{\ell_{0}, \hat{\imath}_{0}}$. Our first result is

Theorem 2. Assume that the initial data $\left(v_{\varepsilon}(0)\right)_{0<\varepsilon<1}$ satisfy conditions $\left(\mathrm{H}_{0}\right),\left(\mathrm{H}_{1}\right)$, and $\left(\mathrm{H}_{\min }\right)$, and let $0<S_{\max } \leq+\infty$ denote the maximal time of existence for the system $\mathcal{S}_{\ell_{0}, \hat{l}_{0}}$ with initial data $a_{k}(0)=a_{k}^{0}$. Then, for $0<s<S_{\max }$,

$$
\mathfrak{v}_{\varepsilon}(s) \longrightarrow v^{\star}\left(\ell_{0}, \hat{\imath}_{0},\left\{a_{k}(s)\right\}\right)
$$

in $L_{\mathrm{loc}}^{\infty}\left(\mathbb{R} \backslash \cup_{k=1}^{\ell_{0}}\left\{a_{k}(s)\right\}\right)$, as $\varepsilon \rightarrow 0$. In particular,

$$
\mathfrak{D}_{\varepsilon}(s) \longrightarrow \cup_{k=1}^{\ell_{0}}\left\{a_{k}(s)\right\}
$$

locally in the sense of the Hausdorff distance, as $\varepsilon \rightarrow 0$.

We consider now the more general situation where $\left(\mathrm{H}_{\min }\right)$ is not verified, and for $1 \leq k \leq \ell_{0}$ we denote by $m_{k}^{0}$ the algebraic multiplicity of $a_{k}^{0}$, namely we set

$$
m_{k}^{0}=\hat{\imath}\left(k+\frac{1}{2}\right)-\hat{\imath}\left(k-\frac{1}{2}\right) .
$$

The case $m_{k}^{0}=0$ corresponds to ghost fronts, whereas $\left|m_{k}^{0}\right| \geq 2$ corresponds to multiple fronts. The total number of fronts that will eventually emerge from such initial data is given by

$$
\ell_{1}=\sum_{k=1}^{\ell_{0}}\left|m_{k}^{0}\right|
$$


and their ordering is obtained by splitting multiple fronts according to the order in $\Sigma$. More precisely, we define the function $\hat{\imath}_{1}$ by

$$
\left\{\begin{array}{l}
\hat{\imath}_{1}\left(\frac{1}{2}\right)=\hat{\imath}_{0}\left(\frac{1}{2}\right), \\
\hat{\imath}_{1}\left(M_{k}^{0}+p+\frac{1}{2}\right)=\hat{\imath}_{0}\left(k+\frac{1}{2}\right)+p, \text { for } p=0, \ldots,\left|m_{k}^{0}\right|-1 \text { if } m_{k}^{0}>0 \\
\hat{\imath}_{1}\left(M_{k}^{0}+p+\frac{1}{2}\right)=\hat{\imath}_{0}\left(k+\frac{1}{2}\right)-p, \text { for } p=0, \ldots,\left|m_{k}^{0}\right|-1 \text { if } m_{k}^{0}<0
\end{array}\right.
$$

where $k=1, \cdots, \ell_{0}$ and $M_{k}^{0}:=\sum_{k=1}^{k-1}\left|m_{k}^{0}\right|$. We say that $\left(\ell_{1}, \hat{\imath}_{1}\right)$ is the splitting of $\left(\ell_{0}, \hat{\imath}_{0}\right)$.

Definition 1. A splitting solution of $(\mathcal{S})$ with initial data $\left(\ell_{0}, \hat{\imath}_{0},\left\{a_{k}^{0}\right\}\right)$ on the interval $[0, S)$ is a solution $a \equiv\left(a_{1}, \cdots, a_{\ell_{1}}\right):(0, S) \rightarrow \mathbb{R}^{\ell_{1}}$ of $\left(\mathcal{S}_{\ell_{1}, \hat{\imath}_{1}}\right)$ such that

$$
\lim _{s \rightarrow 0^{+}} a_{k}(s)=a_{j}^{0} \quad \text { for } \quad k=M_{j}^{0}, \cdots, M_{j}^{0}+\left|m_{j}^{0}\right|-1,
$$

for any $j=1, \cdots, \ell_{0}$, where $\left(\ell_{1}, \hat{\imath}_{1}\right)$ is the splitting of $\left(\ell_{0}, \hat{\imath}_{0}\right)$.

We are now in position to complete Theorem 2 by relaxing assumption $\left(\mathrm{H}_{\min }\right)$.

Theorem 3. Assume that the initial data $\left(v_{\varepsilon}^{0}\right)_{0<\varepsilon<1}$ satisfy conditions $\left(\mathrm{H}_{0}\right)$ and $\left(\mathrm{H}_{1}\right)$. Then there exists a subsequence $\varepsilon_{n} \rightarrow 0$, and a splitting solution of $(\mathcal{S})$ with initial data $\left(\ell_{0}, \hat{\imath}_{0},\left\{a_{k}^{0}\right\}\right)$, defined on its maximal time of existence $\left[0, S_{\max }\right)$, and such that for any $0<s<S_{\max }$

$$
\mathfrak{v}_{\varepsilon_{n}}(s) \longrightarrow v^{\star}\left(\ell_{1}, \hat{\imath}_{1},\left\{a_{k}(s)\right\}\right)
$$

in $L_{\mathrm{loc}}^{\infty}\left(\mathbb{R} \backslash \cup_{k=1}^{\ell_{1}}\left\{a_{k}(s)\right\}\right)$, as $n \rightarrow+\infty$. In particular,

$$
\mathfrak{D}_{\varepsilon_{n}}(s) \longrightarrow \cup_{j=1}^{\ell_{1}}\left\{a_{k}(s)\right\}
$$

locally in the sense of the Hausdorff distance, as $n \rightarrow+\infty$.

Remark 1. Local existence of splitting solutions can be established in different ways (including in particular using Theorem 3 !); to our knowledge, uniqueness is not known, unless of course if $\left|m_{k}^{0}\right| \leq 1$ for all $k$.

So far, our results are constrained by the maximal time of existence $S_{\max }$ of the differential equation $(\mathcal{S})$, which is related to the occurrence of collisions. To pursue the analysis past collisions, we first briefly discuss some properties of the system of equations $(\mathcal{S})$, we refer to Appendix B for more details. The system $(\mathcal{S})$ describes nearest neighbor interactions with an interaction law of the form $\pm d^{-(\omega+1)}, d$ standing for the distance between fronts. The sign of the interactions is crucial, since the system may contain both repulsive forces leading to spreading and attractive forces leading to collisions, yielding the maximal time of existence $S_{\max }$. In order to take signs into account, we set

$$
\epsilon_{k+\frac{1}{2}}=\operatorname{sign}\left(\Gamma_{k+\frac{1}{2}}\right)=-\dagger_{k} \dagger_{k+1} \text {, for } k=0, \cdots, \ell_{0}-1 .
$$

The case $\epsilon_{k+\frac{1}{2}}=-1$ corresponds to repulsive forces between $a_{k}$ and $a_{k+1}$, whereas the case $\epsilon_{k+\frac{1}{2}}=+1$ corresponds to attractive forces between $a_{k}$ and $a_{k+1}$, leading to collisions. As 
a matter of fact, in this last case $a_{k+1}$ corresponds to the anti-front of $a_{k}$. In order to describe the magnitude of the forces, we introduce the subsets $J^{ \pm}$of $\left\{1, \cdots, \ell_{0}\right\}$ defined by $J^{ \pm}=\left\{k \in\left\{1, \cdots, \ell_{0}-1\right\}\right.$, such that $\left.\epsilon_{k+\frac{1}{2}}=\mp 1\right\}$ and the quantities

$$
\left\{\begin{array}{l}
\boldsymbol{\delta}_{a}(s)=\inf \left\{\left|a_{k}(s)-a_{k+1}(s)\right|, \text { for } k \in 1, \cdots, \ell_{0}-1\right\} \\
\boldsymbol{\delta}_{a}^{ \pm}(s)=\inf \left\{\left|a_{k}(s)-a_{k+1}(s)\right|, \text { for } k \in J^{ \pm}\right\}
\end{array}\right.
$$

Proposition 1. There are positive constants $\mathcal{S}_{1}, \mathcal{S}_{2}, \mathcal{S}_{3}$ and $\mathcal{S}_{4}$ depending only on the coefficients of the equation $(\mathcal{S})$, such that for any time $s \in\left[0, S_{\max }\right)$ we have

$$
\left\{\begin{array}{l}
\boldsymbol{\delta}_{a}^{+}(s) \geq\left(\mathcal{S}_{1} s+\mathcal{S}_{2} \boldsymbol{\delta}_{a}^{+}(0)^{\omega+2}\right)^{\frac{1}{\omega+2}} \\
\boldsymbol{\delta}_{a}^{-}(s) \leq\left(\mathcal{S}_{3} \boldsymbol{\delta}_{a}^{-}(0)^{\omega+2}-\mathcal{S}_{4} t\right)^{\frac{1}{\omega+2}}
\end{array}\right.
$$

If for every $k=1, \cdots, \ell_{0}$ we have $\epsilon_{k+\frac{1}{2}}=-1$, then $S_{\max }=+\infty$. Otherwise, we have the estimate

$$
S_{\max } \leq \frac{\mathcal{S}_{3}}{\mathcal{S}_{4}}\left(\boldsymbol{\delta}_{a}^{-}(0)\right)^{\omega+2} \equiv \mathcal{K}_{0}\left(\boldsymbol{\delta}_{a}^{-}(0)\right)^{\omega+2} .
$$

This result shows that the maximal time of existence for solutions to $(\mathcal{S})$ is related to the value of $\boldsymbol{\delta}_{a}^{-}(0)$, the minimal distance between fronts and anti-fronts at time 0 . By the semigroup property, the same can be said about $\boldsymbol{\delta}_{a}^{-}(s)$, namely

$$
S_{\max }-s \lesssim \boldsymbol{\delta}_{a}^{-}(s)^{\omega+2} \text {. }
$$

On the other hand, in view of $(\mathcal{S}), \boldsymbol{\delta}_{a}^{-}(s)$ provides an upper bound for the speeds $\dot{a}_{k}(s)$ in case of collision, namely

$$
\left|\frac{d}{d s} a_{k}(s)\right| \lesssim \delta_{a}^{-}(s)^{-(\omega+1)} .
$$

It follows that

$$
\int_{0}^{S_{\max }}\left|\frac{d}{d s} a_{k}(s)\right| d s \lesssim \int_{0}^{S_{\max }}\left(S_{\max }-s\right)^{-\frac{\omega+1}{\omega+2}} d s<+\infty
$$

and therefore that the trajectories are absolutely continuous up to the collision time. Also, since $\boldsymbol{\delta}_{a}^{+}$remains bounded from below by a positive constant, each front can only enter in collision with its anti-front (but there could be multiple copies of both). From a heuristic point of view, it is therefore rather simple to extend solutions past the collision time: it suffices to remove the colliding pairs from the collection of points, so that the total number of points has been decreased by an even number. More precisely, we have

Corollary 1. Let $\ell_{1}, \hat{\imath}_{1}, a \equiv\left(a_{1}, \cdots, a_{\ell_{1}}\right)$ and $S_{\max }$ be as in Theorem 3. Then, there exist $\ell_{2} \in \mathbb{N}$ such that $\ell_{1}-\ell_{2} \in 2 \mathbb{N}_{*}$, and there exist $\ell_{2}$ points $b_{1}<\cdots<b_{\ell_{2}}$ such that for all $k=1, \cdots, \ell_{1}$

$$
\lim _{s \rightarrow S_{\max }^{-}} a_{k}(s)=b_{j(k)} \quad \text { for some } j(k) \in\left\{1, \cdots, \ell_{2}\right\} .
$$

Moreover, if we set $\hat{\imath}_{2}\left(\frac{1}{2}\right)=\hat{\imath}_{1}\left(\frac{1}{2}\right)$ and

$$
\hat{\imath}_{2}\left(q+\frac{1}{2}\right)=\hat{\imath}_{1}\left(k(q)+\frac{1}{2}\right) \quad \text { where } \quad k(q)=\max \left\{k \in\left\{1, \cdots, \ell_{1}\right\} \text { s.t. } j(k)=q\right\},
$$


for $q=1, \cdots, \ell_{2}$, then

$$
\hat{\imath}_{2}\left(q+\frac{1}{2}\right)-\hat{\imath}_{2}\left(q-\frac{1}{2}\right) \in\{+1,-1,0\}
$$

for all $q=1, \cdots, \ell_{2}$.

We stress than Corollary 1 is obtained from Theorem 3 using only properties of the system of ODE's $(\mathcal{S})$, in particular Proposition 1.

We are now in position to state our last result, namely

Theorem 4. Under the assumptions of Theorem 3, we have as $n \rightarrow+\infty$,

$$
\mathfrak{v}_{\varepsilon_{n}}\left(S_{\max }\right) \longrightarrow v^{\star}\left(\ell_{2}, \hat{\imath}_{2},\left\{b_{k}\right\}\right) \quad \text { in } \quad L_{\text {loc }}^{\infty}\left(\mathbb{R} \backslash \cup_{k=1}^{\ell_{2}}\left\{b_{k}\right\}\right),
$$

where $\ell_{2}, \hat{\imath}_{2}$ and $b_{1}<\cdots<b_{\ell_{2}}$ are given by Corollary 1. In particular the sequence $\left(\mathfrak{v}_{\varepsilon_{n}}\left(S_{\max }\right)\right)_{n \in \mathbb{N}}$, considered as initial data, satisfies the assumptions $\left(\mathrm{H}_{0}\right)$ and $\left(\mathrm{H}_{1}\right)$ with $\ell_{0}:=\ell_{2}$ and $\left\{a_{k}^{0}\right\}:=\left\{b_{k}^{0}\right\}$.

We may therefore apply Theorem 3 to the sequence of initial data $\left(\mathfrak{v}_{\varepsilon_{n}}\left(S_{\max }\right)\right)_{n \in \mathbb{N}}$, and therefore, using the semi-group property of (1), extend the analysis past $S_{\max }$. Notice that since the multiplicites given by $\hat{\imath}_{2}$ are either equal to \pm 1 or 0 , no further subsequences are needed to pass through the collision times. Finally, since the total number of fronts is decreased at least by 2 at each collision times, the latter are finitely many.

Some comments on the results. Motion of fronts for one-dimensional scalar reactiondiffusion equations has already a quite long history. Most of the efforts have been devoted until recently to the case where the potential possesses only two wells with non vanishing second derivative: such potentials are often referred to as Allen-Cahn potentials. Under suitable preparedness assumptions on the initial datum, the precise motion law for the fronts has been derived by Carr and Pego in their seminal work [9] (see also Fusco and Hale [10]). They showed that the front points are moved, up to the first collision time, according to a first order differential equation of nearest neighbor interaction type, with interactions terms proportional to $\exp \left(-\varepsilon^{-1}\left(a_{k+1}^{\varepsilon}(t)-a_{k}^{\varepsilon}(t)\right)\right)$. These results present substantial differences with the results in the present paper, in particular we wish to emphasize the following points:

- only attractive forces leading eventually to the annihilation of fronts with anti-fronts forces are present.

- the equation is not renormalizable. Indeed, the various forces $\exp \left(-\varepsilon^{-1}\left(a_{k+1}^{\varepsilon}(t)-a_{k}^{\varepsilon}(t)\right)\right)$ for different values of $k$ may be of very different orders of magnitude, and hence not commensurable.

Besides this, the essence of their method is quite different: it relies on a careful study of the linearized problem around the stationary front, in particular from the spectral point of view. This type of approach is also sometimes termed the geometric approach (see e.g. [8]). At least two other methods have been applied successfully on the Allen-Cahn equation. Firstly, the method of subsolutions and supersolutions turns out to be extremely powerful and allowed to handle larger classes of initial data and also to extend the analysis past collisions: this is for instance achieved by Chen in [8]. Another direction is given by the global energy approach initiated by Bronsard and Kohn [7]. We refer to [4] for a more references on these methods. 
Several ideas and concepts presented here are influenced by our earlier work on the motion of vortices in the two-dimensional parabolic Ginzburg-Landau equation [2,3]. As a matter of fact, this equation yields another remarkable example of renormalizable slow motion, as proved by Lin or Jerrard and Soner $([13,11])$. Our interest in the questions studied in this paper was certainly driven by the possibility of finding an analogous situation in one space dimension.

This paper belongs to series of papers we have written on the slow motion phenomenon for reaction-diffusion equation of gradient type with mutiple-wells (see $[4,5,6]$ ). Common to these papers is a general approach based on the following ingredients:

- A localized version of the energy identity (see subsection 1.3). Fronts are then handled as concentration points of the energy, so that the evolution of local energies yields also the motion of fronts. Besides dissipation, this localized energy identity contains a flux term, involving the discrepancy function, which has a simple interpretation for stationary solutions. Using test functions which are affine near the fronts, the flux term does not see the core of the front, only its tail.

- Parabolic estimates away from the fronts.

- Handling the time derivative as a perturbation of the one-dimensional elliptic equations, allowing hence elementary tools as Gronwall's identities.

Parallel to this paper, we are also revisiting the scalar non-degenerate case in [6], considering in particular the case were there are more than two wells, leading as mentioned to repulsive forces which are not present in the Allen-Cahn case. Several tools are shared by the two papers, for instance we rely on related definitions and properties of regularized fronts, and the properties of the ordinary differential equations are quite similar. From a technical point of view differences appear at the level of the magnitudes of energies as well as of the parameter $\delta$ involved in the definition of regular fronts, and more crucially on the nature of the parabolic estimates off the front sets. Whereas in [6] we rely essentially on linear estimates, in the degenerate case considered here our estimates are truly non-linear, obtained mainly through an extensive use of the comparison principle.

Finally, it is presumably worthwhile to mention that the situation in higher dimension is very different: the dynamics is dominated by mean-curvature effects. The phenomena considered in the present paper are therefore of lower order, and do not appear in the limiting equations.

Among the problems left open in our paper, we would like to emphasize again the question of uniqueness of splitting solutions for $(\mathcal{S})$, as well as the possibility to interpret our convergence results in terms of Gamma-limit involving a renormalized energy (see e.g [15] for related results on the Ginzburg-Landau equation).

\subsection{Regularized fronts}

The notion of regularized fronts is central in our description of the dynamics of equation $(\mathrm{PGL})_{\varepsilon}$. It is aimed to describe in a quantitative way chains of stationary solutions which are well-separated and suitably glued together. It also allows to pass from front sets to front points, a notion which is more accurate and requires therefore improved estimates. Recall 
first that for $i \in\{1, \cdots, \mathfrak{q}-1\}$, there exist a unique (up to translations) solution $\zeta_{i}^{+}$to the stationary equation with $\varepsilon=1$,

$$
-v_{x x}+V^{\prime}(v)=0 \text { on } \mathbb{R}
$$

with, as conditions at infinity, $v(-\infty)=\sigma_{i}$ and $v(+\infty)=\sigma_{i+1}$. Set, for $i \in\{1, \cdots, \mathfrak{q}-1\}$, $\zeta_{i}^{-}(\cdot) \equiv \zeta_{i}(-\cdot)$, so that $\zeta_{i}^{-}$is the unique (up to translations) solution to (21) such that $v(+\infty)=\sigma_{i}$ and $v(-\infty)=\sigma_{i+1}$. A remarkable yet elementary fact, related to the scalar nature of the equation, is that there are no other non trivial finite energy solutions to equation (21) than the solutions $\zeta_{i}^{ \pm}$and their translates: in particular there are no solutions connecting minimizers which are not nearest neighbors. For $i=1, \cdots, \mathfrak{q}-1$, we fix a point $z_{i}$ in the interval $\left(\sigma_{i}, \sigma_{i+1}\right)$ where the potential $V$ restricted to $\left[\sigma_{i}, \sigma_{i+1}\right]$ achieves its maximum and we set $\mathcal{Z}=\left\{z_{1}, \cdots, z_{\mathfrak{q}-1}\right\}$. Again, since we consider only the one-dimensional scalar case, any solution $\zeta_{i}$ takes once and only once the value $z_{i}$.

We next describe a local notion of well-preparedness ${ }^{4}$. For an arbitrary $r>0$, we denote by $\mathrm{I}_{r}$ the interval $[-r, r]$.

Definition 2. Let $\mathrm{L}>0$ and $\delta>0$. We say that a map $u$ verifying $\left(H_{0}\right)$ satisfies the preparedness assumption $\mathcal{W P}_{\varepsilon}^{\mathrm{L}}(\delta)$ if the following two conditions are fulfilled:

- $\left(\operatorname{WPI}_{\varepsilon}^{\mathrm{L}}(\delta)\right) \quad$ We have

$$
\mathcal{D}(u) \cap \mathrm{I}_{2 \mathrm{~L}} \subset \mathrm{I}_{\mathrm{L}}
$$

and there exists a collection of points $\left\{a_{k}\right\}_{k \in J}$ in $\mathrm{I}_{\mathrm{L}}$, with $J=\{1, \cdots, \ell\}$, such that

$$
\mathcal{D}(u) \cap \mathrm{I}_{2 \mathrm{~L}} \subset \underset{k \in J}{\cup} I_{k}, \quad \text { where } I_{k}=\left[a_{k}-\delta, a_{k}+\delta\right] .
$$

For $k \in J$, there exist a number $i(k) \in\{1, \cdots, \mathfrak{q}-1\}$ such that $u\left(a_{k}\right)=z_{i(k)}$ and $a$ symbol $\dagger_{k} \in\{+,-\}$ such that

$$
\left\|u(\cdot)-\zeta_{i(k)}^{\dagger k}\left(\frac{\cdot-a_{k}}{\varepsilon}\right)\right\|_{C_{\varepsilon}^{1}\left(I_{k}\right)} \leq \exp \left(-\frac{\delta}{\varepsilon}\right)
$$

where $\|u\|_{C_{\varepsilon}^{1}\left(I_{k}\right)}=\|u\|_{L^{\infty}\left(I_{k}\right)}+\varepsilon\left\|u^{\prime}\right\|_{L^{\infty}\left(I_{k}\right)}$.

- $\left(\mathrm{WPO}_{\varepsilon}^{\mathrm{L}}(\delta)\right) \quad$ Set $\Omega_{\mathrm{L}}=\mathrm{I}_{2 \mathrm{~L}} \backslash \bigcup_{k=1}^{\ell} I_{k}$. We have the energy estimate

$$
\int_{\Omega_{\mathrm{L}}} e_{\varepsilon}(u(x)) d x \leq \mathrm{C}_{\mathrm{w}} M_{0}\left(\frac{\varepsilon}{\delta}\right)^{\omega} .
$$

In the above definition $\mathrm{C}_{\mathrm{w}}>0$ denotes a constant, whose exact value is fixed once for all by Proposition 2.1 below, and which depends only on $V$. Condition $\mathrm{WPI}_{\varepsilon}^{\mathrm{L}}(\delta)$ corresponds to an inner matching of the map with stationary fronts, it is only really meaningful if $\delta>>\varepsilon$. In the sequel we always assume that

$$
\frac{L}{2} \geq \delta \geq \alpha_{1} \varepsilon
$$

\footnotetext{
${ }^{4} \mathrm{By}$ local, we mean with respect to the interval $[-\mathrm{L}, \mathrm{L}]$. In contrast the related notion introduced in $[6]$ is global on the whole of $\mathbb{R}$
} 
where $\alpha_{1}$ is larger than the $\alpha_{0}$ of Theorem 1 and also sufficiently large so that if $\operatorname{WPI}_{\varepsilon}^{\mathrm{L}}(\delta)$ holds then the points $a_{k}$ and the indices $i(k)$ and $\dagger_{k}$ are uniquely and therefore unambigously determined and the intervals $I_{k}$ are disjoints. In particular, the quantity $d_{\min }^{\varepsilon, \mathrm{L}}(s)$, defined by

$$
d_{\min }^{\varepsilon, \mathrm{L}}(s):=\min \left\{a_{k+1}^{\varepsilon}(s)-a_{k}^{\varepsilon}(s), \quad k=1, \cdots, \ell(s)-1\right\}
$$

if $\ell(s) \geq 2$, and $d_{\min }^{\varepsilon, \mathrm{L}}(s)=2 \mathrm{~L}$ otherwise, satisfies $d_{\min }^{\varepsilon, \mathrm{L}}(s) \geq 2 \delta$. Condition $\mathrm{WPO}_{\varepsilon}^{\mathrm{L}}(\delta)$ is in some weak sense an outer matching: it is crucial for some of our energy estimates and its form is motivated by energy decay estimates for stationary solutions. Note that condition $\operatorname{WPI}_{\varepsilon}^{\mathrm{L}}(\delta)$ makes sense on its own, whereas condition $\mathrm{WPO}_{\varepsilon}^{\mathrm{L}}(\delta)$ only makes sense if condition $\mathrm{WPI}_{\varepsilon}^{\mathrm{L}}(\delta)$ is fullfilled. Note also that the larger $\delta$ is, the stronger condition $\operatorname{WPI}_{\varepsilon}^{\mathrm{L}}(\delta)$ is. The same is not obviously true for condition $\mathrm{WPO}_{\varepsilon}^{\mathrm{L}}(\delta)$, since the set of integration $\Omega_{\mathrm{L}}$ increases with $\delta$. As a matter of fact, the constant $\mathrm{C}_{\mathrm{w}}$ in $(25)$ is chosen sufficiently $\operatorname{big}^{5}$ so that $\mathrm{WPO}_{\varepsilon}^{\mathrm{L}}(\delta)$ also becomes stronger when $\delta$ is larger. We next specify Definition 2 for the maps $x \mapsto \mathfrak{v}_{\varepsilon}(x, s)$.

Definition 3. For $s \geq 0$, we say that the assumption $\mathcal{W P}_{\varepsilon}^{\mathrm{L}}(\delta, s)\left(\right.$ resp. $\left.\mathrm{WPI}_{\varepsilon}^{\mathrm{L}}(\delta, s)\right)$ holds if the map $x \mapsto \mathfrak{v}_{\varepsilon}(x, s)$ satisfies $\mathcal{W P}_{\varepsilon}^{\mathrm{L}}(\delta)$ (resp. $\mathrm{WPI}_{\varepsilon}^{\mathrm{L}}(\delta)$ ).

When assumption $\operatorname{WPI}_{\varepsilon}^{\mathrm{L}}(\delta, s)$ holds, then all symbols will be indexed according to $s$. In particular, we write ${ }^{6} \ell(s)=\ell, J(s)=J$, and $a_{k}^{\varepsilon}(s)=a_{k}$. The points $a_{k}^{\varepsilon}(s)$ for $k \in J(s)$, are now termed the front points. Whereas in [6] we are able, due to parabolic regularization, to establish under suitable conditions that $\mathcal{W P}_{\varepsilon}^{\mathrm{L}}(\delta, s)$ is fulfilled for length of the same order as the minimal distance between the front points, this is not the case in the present situation. More precisely, two orders of magnitude for $\delta$ will be considered, namely

$$
\delta_{\log }^{\varepsilon}=\frac{1}{\rho_{\mathrm{w}}} \varepsilon\left|\log \left(4 M_{0}^{2} \frac{\varepsilon}{\mathrm{L}}\right)\right| \quad \text { and } \quad \delta_{\log \log }^{\varepsilon}=\frac{\omega}{\rho_{\mathrm{w}}} \varepsilon \log \left(\frac{1}{\rho_{\mathrm{w}}}\left|\log \left(4 M_{0}^{2} \frac{\varepsilon}{\mathrm{L}}\right)\right|\right) .
$$

In (27), the constant $\rho_{\mathrm{w}}$ (given by Lemma 2.4 below) depends only on $V$. The main property for our purposes is that $\delta_{\log \log }^{\varepsilon} / \varepsilon$ and $\delta_{\log }^{\varepsilon} / \delta_{\log \log }^{\varepsilon}$ both tend to $+\infty$ as $\varepsilon / \mathrm{L}$ tends to 0 .

In many places, it is useful to rely on a slightly stronger version of the confinement condition (22), which we assume to hold on some interval of time. More precisely, for positive L, $S$ we consider the condition

$$
\mathfrak{D}_{\varepsilon}(s) \cap \mathrm{I}_{4 \mathrm{~L}} \subset \mathrm{I}_{\mathrm{L}}, \quad \forall 0 \leq s \leq S .
$$

where the constant $\mathrm{C}_{e}$ is defined in Proposition 2 here below. For given $\mathrm{L}_{0}>0$ and $S>0$, it follows easily from assumption $\left(\mathrm{H}_{1}\right)$ and Theorem 1 that there exists $\mathrm{L} \geq \mathrm{L}_{0}$ for which the first condition in $\left(\mathcal{C}_{\mathrm{L}, S}\right)$ is satisfied. Under condition $\left(\mathcal{C}_{\mathrm{L}, S}\right)$, the estimate

$$
\mathcal{E}_{\varepsilon}\left(\mathfrak{v}_{\varepsilon}(s), \mathrm{I}_{3 \mathrm{~L}} \backslash \mathrm{I}_{\frac{3}{2} \mathrm{~L}}\right) \leq \mathrm{C}_{e}\left(\frac{\varepsilon}{\mathrm{L}}\right)^{\omega}, \quad \forall s \in\left[\varepsilon^{\omega} \mathrm{L}^{2}, S\right],
$$

follows from the following regularizing effect, which was obtained in [5]:

\footnotetext{
${ }^{5}$ In view of $\mathrm{WPI}_{\varepsilon}^{\mathrm{L}}(\delta)$, how big it needs to be is indeed related to energy decay estimates for the fronts $\zeta_{i}$.

${ }^{6}$ In principle and at this stage, all those symbols depend also upon $\varepsilon$. Since eventually $\ell$ and $J$ will be $\varepsilon$-independent, at least for $\varepsilon$ sufficiently small, we do not explicitly index them with $\varepsilon$.
} 
Proposition $2([5])$. Let $v_{\varepsilon}$ be a solution to $(P G L)_{\varepsilon}$, let $x_{0} \in \mathbb{R}, r>0$ and $0 \leq s_{0}<S$ be such that

$$
\mathfrak{v}_{\varepsilon}(y, s) \in B\left(\sigma_{i}, \mu_{0}\right) \quad \text { for all }(y, s) \in\left[x_{0}-r, x_{0}+r\right] \times\left[s_{0}, S\right],
$$

for some $i \in\{1, \cdots, \mathfrak{q}\}$. Then we have for $s_{0}<s \leq S$

$$
\varepsilon^{-\omega} \int_{x_{0}-3 r / 4}^{x_{0}+3 r / 4} e_{\varepsilon}\left(\mathfrak{v}_{\varepsilon}(x, s)\right) d x \leq \frac{1}{10} \mathrm{C}_{e}\left(1+\left(\frac{\varepsilon^{\omega} r^{2}}{s-s_{0}}\right)^{\frac{\theta}{\theta-1}}\right)\left(\frac{1}{r}\right)^{\omega}
$$

as well as

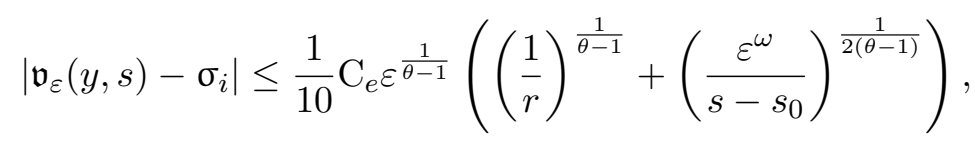

for $y \in\left[x_{0}-3 r / 4, x_{0}+3 r / 4\right]$, where the constant $C>0$ depends only on $V$.

Our first ingredient is

Proposition 3. There exists $\alpha_{1}>0$, depending only on $M_{0}$ and $V$, such that if $L \geq \alpha_{1} \varepsilon$ and if $\left(\mathcal{C}_{\mathrm{L}, S}\right)$ holds, then each subinterval of $[0, S]$ of length $\varepsilon^{\omega+2}(\mathrm{~L} / \varepsilon)$ contains at least one time $s$ for which $\mathcal{W P}_{\varepsilon}^{\mathrm{L}}\left(\delta_{\log }^{\varepsilon}, s\right)$ holds.

The idea behind Proposition 3 is that, $(\mathrm{PGL})_{\varepsilon}$ being a gradient flow, on a sufficiently large interval of time one may find some time where the dissipation of energy is small. Using elliptic tools, and viewing the time derivative as a forcing term, one may then establish property $\mathcal{W} \mathcal{P}_{\varepsilon}^{\mathrm{L}}\left(\delta_{\log }^{\varepsilon}, s\right)$ (see Section 2 and Section 3).

The next result expresses the fact that the equation preserves to some extent the wellpreparedness assumption.

Proposition 4. Assume that $\left(\mathcal{C}_{\mathrm{L}, S}\right)$ holds, that $\varepsilon^{\omega} \mathrm{L}^{2} \leq s_{0} \leq S$ is such that $\mathcal{W} \mathcal{P}_{\varepsilon}^{\mathrm{L}}\left(\delta_{\log }^{\varepsilon}, s_{0}\right)$ holds, and assume moreover that

$$
d_{\min }^{\varepsilon, \mathrm{L}}\left(s_{0}\right) \geq 16\left(\frac{\mathrm{L}}{\rho_{0} \varepsilon}\right)^{\frac{1}{\omega+2}} \varepsilon .
$$

Then $\mathcal{W P}_{\varepsilon}^{\mathrm{L}}\left(\delta_{\log \log }^{\varepsilon}, s\right)$ holds for all times $s_{0}+\varepsilon^{2+\omega} \leq s \leq \mathcal{T}_{0}^{\varepsilon}\left(s_{0}\right)$, where

$$
\mathcal{T}_{0}^{\varepsilon}\left(s_{0}\right)=\max \left\{s \in\left[s_{0}+\varepsilon^{2+\omega}, S\right] \quad \text { s.t. } \quad d_{\text {min }}^{\varepsilon, \mathrm{L}}\left(s^{\prime}\right) \geq 8\left(\frac{\mathrm{L}}{\rho_{0} \varepsilon}\right)^{\frac{1}{\omega+2}} \varepsilon \quad \forall s^{\prime} \in\left[s_{0}+\varepsilon^{\omega+2}, s\right]\right\} .
$$

For such $s$ we have $J(s)=J\left(s_{0}\right)$ and for any $k \in J\left(s_{0}\right)$ we have $\sigma_{i\left(k \pm \frac{1}{2}\right)}(s)=\sigma_{i\left(k \pm \frac{1}{2}\right)}\left(s_{0}\right)$ and $\dagger_{k}(s)=\dagger_{k}\left(s_{0}\right)$.

Given a family of solution $\left(v_{\varepsilon}\right)_{0<\varepsilon<1}$, we introduce the additional condition

$$
d_{\min }^{*}\left(s_{0}\right) \equiv \liminf _{\varepsilon \rightarrow 0} d_{\min }^{\varepsilon, \mathrm{L}}\left(s_{0}\right)>0,
$$

which makes sense if $\mathcal{W P}_{\varepsilon}^{\mathrm{L}}\left(\alpha_{1} \varepsilon, s_{0}\right)$ holds and expresses the fact that the fronts stay uniformly well-separated. The first step in our proofs, which is stated in Proposition 6 below, is to establish the conclusion of Theorem 2 under this stronger assumptions on the initial datum. From the inclusion (7) and Proposition 4 we will obtain: 
Corollary 2. Assume also that $\mathcal{C}_{\mathrm{L}, S}$ holds, let $s_{0} \in[0, S]$ and assume that $\mathcal{W} \mathcal{P}_{\varepsilon}^{\mathrm{L}}\left(\alpha_{1} \varepsilon, s_{0}\right)$ holds for all $\varepsilon$ sufficiently small and that (33) is satisfied. Then, for $\varepsilon$ sufficiently small,

$$
\mathcal{W P}_{\varepsilon}^{\mathrm{L}}\left(\delta_{\log \log }^{\varepsilon}, s\right) \quad \text { and } \quad d_{\min }^{\varepsilon, \mathrm{L}}(s) \geq \frac{1}{2} d_{\min }^{*}\left(s_{0}\right)
$$

are satisfied for any

$$
s \in I^{\varepsilon}\left(s_{0}\right) \equiv\left[s_{0}+2 \mathrm{~L}^{2} \varepsilon^{\omega}, s_{0}+\rho_{0}\left(\frac{d_{\min }^{*}\left(s_{0}\right)}{8}\right)^{\omega+2}\right] \cap[0, S]
$$

as well as the identities $J(s)=J\left(s_{0}\right), \sigma_{i\left(k \pm \frac{1}{2}\right)}(s)=\sigma_{i\left(k \pm \frac{1}{2}\right)}\left(s_{0}\right)$ and $\dagger_{k}(s)=\dagger_{k}\left(s_{0}\right)$, for any $k \in J\left(s_{0}\right)$.

Hence, the collection of front points $\left\{a_{k}^{\varepsilon}(s)\right\}_{k \in J}$ is well-defined, and the approximating regularized fronts $\zeta_{i(k)}^{\dagger}$ do not depend on $s$ (otherwise than through their position), on the full time interval $I^{\varepsilon}\left(s_{0}\right)$.

\subsection{Paving the way to the motion law}

As in [4], we use extensively the localized version of (1), a tool which turns out to be perfectly adapted to track the evolution of fronts. Let $\chi$ be an arbitrary smooth test function with compact support. Set, for $s \geq 0$,

$$
\mathcal{I}_{\varepsilon}(s, \chi)=\int_{\mathbb{R}} e_{\varepsilon}\left(\mathfrak{v}_{\varepsilon}(x, s)\right) \chi(x) d x .
$$

In integrated form the localized version of the energy identity writes

$$
\mathcal{I}_{\varepsilon}\left(s_{2}, \chi\right)-\mathcal{I}_{\varepsilon}\left(s_{1}, \chi\right)+\int_{s_{1}}^{s_{2}} \int_{\mathbb{R}} \varepsilon^{1+\omega} \chi(x)\left|\partial_{s} \mathfrak{v}_{\varepsilon}(x, s)\right|^{2} d x d s=\varepsilon^{-\omega} \int_{s_{1}}^{s_{2}} \mathcal{F}_{S}\left(s, \chi, v_{\varepsilon}\right) d s,
$$

where the term $\mathcal{F}_{S}$ is given by

$$
\mathcal{F}_{S}\left(s, \chi, \mathfrak{v}_{\varepsilon}\right)=\int_{\mathbb{R} \times\{s\}}\left(\left[\varepsilon \frac{\dot{\mathfrak{v}_{\varepsilon}}}{2}-\frac{V\left(\mathfrak{v}_{\varepsilon}\right)}{\varepsilon}\right] \ddot{\chi}(x)\right) d x \equiv \int_{\mathbb{R} \times\{s\}} \xi_{\varepsilon}\left(\mathfrak{v}_{\varepsilon}(\cdot, s)\right) \ddot{\chi} d x .
$$

The last integral on the left hand side of identity (36) stands for local dissipation, whereas the right hand side second is a flux. The quantity $\xi_{\varepsilon}$ is defined for a scalar function $u$ by

$$
\xi_{\varepsilon}(u) \equiv \varepsilon \frac{\dot{u}^{2}}{2}-\frac{V(u)}{\varepsilon},
$$

and is referred to as the discrepancy term. It is constant for solutions to the stationary equation $-u_{x x}+\varepsilon^{-2} V^{\prime}(u)=0$ on some given interval $I$ and vanishes for finite energy solutions on $I=\mathbb{R}$. Notice that $\left|\xi_{\varepsilon}(u)\right| \leq e_{\varepsilon}(u)$. We set for two given times $s_{2} \geq s_{1} \geq 0$ and $\mathrm{L} \geq 0$

$$
\operatorname{dissip}_{\varepsilon}^{\mathrm{L}}\left[s_{1}, s_{2}\right]=\varepsilon \int_{\mathrm{I}_{\frac{5}{3} \mathrm{~L}} \times\left[s_{1} \varepsilon^{-\omega}, s_{2} \varepsilon^{-\omega}\right]}\left|\frac{\partial v_{\varepsilon}}{\partial t}\right|^{2} d x d t=\varepsilon^{1+\omega} \int_{I_{\frac{5}{3} \mathrm{~L}} \times\left[s_{1}, s_{2}\right]}\left|\frac{\partial \mathfrak{v}_{\varepsilon}}{\partial s}\right|^{2} d x d s .
$$


Identity (36) then yields the estimate, if we assume that $\operatorname{supp} \chi \subset \mathrm{I}_{\frac{5}{3} \mathrm{~L}}$,

$$
\left|\mathcal{I}_{\varepsilon}\left(s_{2}, \chi\right)-\mathcal{I}_{\varepsilon}\left(s_{1}, \chi\right)-\varepsilon^{-\omega} \int_{s_{1}}^{s_{2}} \mathcal{F}_{S}\left(s, \chi, v_{\varepsilon}\right) d s\right| \leq \operatorname{dissip}_{\varepsilon}^{\mathrm{L}}\left[s_{1}, s_{2}\right]\|\chi\|_{L^{\infty}(\mathbb{R})} .
$$

We will show that under suitable assumptions, that the right hand side of (40) is small (see Step 3 in the proof of Proposition 6), so that the term $\varepsilon^{-\omega} \int_{s_{1}}^{s_{2}} \mathcal{F}_{S}\left(s, \chi, v_{\varepsilon}\right) d s$ provides a good approximation of $\mathcal{I}_{\varepsilon}\left(s_{2}, \chi\right)-\mathcal{I}_{\varepsilon}\left(s_{1}, \chi\right)$. On the other hand, it follows from the properties of regularized maps proved in Section 2.2 (see Proposition 2.1 there) that if $\mathcal{W} \mathcal{P}_{\varepsilon}^{\mathrm{L}}\left(\delta_{\log \log }^{\varepsilon}, s\right)$ holds then

$$
\left|\mathcal{I}_{\varepsilon}(s, \chi)-\sum_{k \in J} \chi\left(a_{k}^{\varepsilon}(s)\right) \mathfrak{S}_{i(k)}\right| \leq C M_{0}\left(\left(\frac{\varepsilon}{\delta_{\log \log }^{\varepsilon}}\right)^{\omega}\|\chi\|_{\infty}+\varepsilon\left\|\chi^{\prime}\right\|_{\infty}\right),
$$

where $\mathfrak{S}_{i(k)}$ stands for the energy of the corresponding stationary front. Set

$$
\mathfrak{F}_{\varepsilon}\left(s_{1}, s_{2}, \chi\right) \equiv \varepsilon^{-\omega} \int_{s_{1}}^{s_{2}} \mathcal{F}_{S}\left(s, \chi, \mathfrak{v}_{\varepsilon}\right) d s \equiv \int_{s_{1}}^{s_{2}} \varepsilon^{-\omega} \xi_{\varepsilon}\left(\mathfrak{v}_{\varepsilon}(\cdot, s)\right) \ddot{\chi}(\cdot) d s .
$$

Combining (40) and (41) shows that, if $\mathcal{W P}_{\varepsilon}^{\mathrm{L}}\left(\delta_{\log l o g}^{\varepsilon}, s\right)$ holds for any $s \in\left(s_{1}, s_{2}\right)$, then we have

$$
\begin{aligned}
& \left|\sum_{k \in J}\left[\chi\left(a_{k}^{\varepsilon}\left(s_{2}\right)\right)-\chi\left(a_{k}^{\varepsilon}\left(s_{1}\right)\right)\right] \mathfrak{S}_{i(k)}-\mathfrak{F}_{\varepsilon}\left(s_{1}, s_{2}, \chi\right)\right| \\
\leq & C M_{0}\left(\left(\log \left|\log \frac{\varepsilon}{\mathrm{L}}\right|\right)^{-\omega}\|\chi\|_{\infty}+\varepsilon\left\|\chi^{\prime}\right\|_{\infty}\right)+\operatorname{dissip}_{\varepsilon}^{\mathrm{L}}\left[s_{1}, s_{2}\right]\|\chi\|_{\infty} .
\end{aligned}
$$

If the test function $\chi$ is choosen to be affine near a given front point $a_{k_{0}}$ and zero near the other front points in the collection, then the first term on the left hand side yields a measure of the motion of $a_{k_{0}}$ between times $s_{1}$ and $s_{2}$, whereas the second, namely $\mathfrak{F}_{\varepsilon}\left(s_{1}, s_{2}, \chi\right)$, is hence a good approximation of the measure of this motion, provided we are able to estimate the dissipation $\operatorname{dissip}_{\varepsilon}^{\mathrm{L}}\left(s_{1}, s_{2}\right)$. Our previous discussion suggests that

$$
a_{k_{0}}^{\varepsilon}\left(s_{2}\right)-a_{k_{0}}^{\varepsilon}\left(s_{1}\right) \simeq \frac{1}{\chi^{\prime}\left(a_{k_{0}}^{\varepsilon}\right) \mathfrak{S}_{i\left(k_{0}\right)}} \mathfrak{F}_{\varepsilon}\left(s_{1}, s_{2}, \chi\right) .
$$

It turns out that the computation of $\mathfrak{F}_{\varepsilon}\left(s_{1}, s_{2}, \chi\right)$ can be performed with satisfactory accuracy if the test function $\chi$ is affine (and hence as vanishing second derivatives) close to the front set, this is the object of the next subsections.

\subsection{A first compactness result}

A first step in deriving the motion law for the fronts is to obtain rough bounds from above for both $\operatorname{dissip}_{\varepsilon}^{\mathrm{L}}\left[s_{1}, s_{2}\right]$ and $\mathfrak{F}_{\varepsilon}\left(s_{1}, s_{2}, \chi\right)$. To obtain these, and under the assumptions of Corollary 2 , notice that if $\ddot{\chi}$ vanishes on the set $\left\{a_{k}^{\varepsilon}\left(s_{0}\right)\right\}_{k \in J}+\left[-d_{\min }^{*}\left(s_{0}\right) / 4, d_{\min }^{*}\left(s_{0}\right) / 4\right]$, then from the inequality $\left|\xi_{\varepsilon}(u)\right| \leq e_{\varepsilon}(u)$, from Corollary 2 and from (30) of Proposition 2, we derive that for $s_{1} \leq s_{2}$ in $I^{\varepsilon}\left(s_{0}\right)$,

$$
\left|\mathfrak{F}_{\varepsilon}\left(s_{1}, s_{2}, \chi\right)\right| \leq C d_{\min }^{*}\left(s_{0}\right)^{-\omega}\|\ddot{\chi}\|_{L^{\infty}(\mathbb{R})}\left(s_{2}-s_{1}\right) .
$$

Going back to (36), and choosing the test fucntion $\chi$ so that $\chi \equiv 1$ on $\mathrm{I}_{\frac{5}{3} \mathrm{~L}}$ with compact support on $\mathrm{I}_{2 \mathrm{~L}}$, estimate (43) combined with (41) yields in turn a first rough upper bound on the dissipation $\operatorname{dissip}_{\varepsilon}^{\mathrm{L}}\left[s_{1}, s_{2}\right]$. Combining these estimates we will otain 
Proposition 5. Under the assumptions of Corollary 2, for $s_{1} \leq s_{2} \in I^{\varepsilon}\left(s_{0}\right)$ we have

$$
\left|a_{k}^{\varepsilon}\left(s_{1}\right)-a_{k}^{\varepsilon}\left(s_{2}\right)\right| \leq C\left(d_{\min }^{*}\left(s_{0}\right)^{-(\omega+1)}\left(s_{2}-s_{1}\right)+M_{0}\left(\left(\log \left|\log \frac{\varepsilon}{\mathrm{L}}\right|\right)^{-\omega} d_{\min }^{*}\left(s_{0}\right)+\varepsilon\right)\right) .
$$

As an easy consequence, we deduce the following compactness property, setting

$$
I^{*}\left(s_{0}\right)=\left(s_{0}, s_{0}+\rho_{0}\left(\frac{d_{\min }^{*}\left(s_{0}\right)}{8}\right)^{\omega+2}\right) \cap(0, S) .
$$

Corollary 3. Under the assumptions of Corollary 2, there exist a subsequence $\left(\varepsilon_{n}\right)_{n \in \mathbb{N}}$ converging to 0 such that for any $k \in J$ the function $a_{k}^{\varepsilon_{n}}(\cdot)$ converges uniformly on any compact interval of $I^{*}\left(s_{0}\right)$ to a lipschitz continuous function $a_{k}(\cdot)$.

\subsection{Refined estimates off the front set and the motion law}

In order to derive the precise motion law, we have to provide an accurate asymptotic value for the discrepancy term off the front set. In other words, for a given index $k \in J$ we need to provide a uniform limit of the function $\varepsilon^{-\omega} \xi_{\varepsilon}$ near the points

$$
a_{k+\frac{1}{2}}^{\varepsilon}(s) \equiv \frac{a_{k}^{\varepsilon}(s)+a_{k+1}^{\varepsilon}(s)}{2} \text { and } a_{k-\frac{1}{2}}^{\varepsilon}(s) \equiv \frac{a_{k-1}^{\varepsilon}(s)+a_{k}^{\varepsilon}(s)}{2} .
$$

We notice first that $\mathfrak{v}_{\varepsilon}$ takes values close to $\sigma_{i\left(k+\frac{1}{2}\right)}$ near $a_{k+\frac{1}{2}}^{\varepsilon}(s)$. In view of estimate (30), we introduce the functions

$$
\mathfrak{w}_{\varepsilon}(\cdot, s)=\mathfrak{w}_{\varepsilon}^{k}(\cdot, s)=\mathfrak{v}_{\varepsilon}-\sigma_{i\left(k+\frac{1}{2}\right)} \text { and } \mathfrak{W}_{\varepsilon}=\mathfrak{W}_{\varepsilon}^{k} \equiv \varepsilon^{-\frac{1}{\theta-1}} \mathfrak{w}_{\varepsilon}^{k}=\varepsilon^{-\frac{1}{\theta-1}}\left(\mathfrak{v}_{\varepsilon}-\sigma_{i\left(k+\frac{1}{2}\right)}\right) .
$$

As a consequence of inequality (31) and Corollary 2 we have the uniform bound:

Lemma 2. Under the assumptions of Corollary 2, we have

$$
\left|\mathfrak{W}_{\varepsilon}(x, s)\right| \leq C(d(x, s))^{-\frac{1}{\theta-1}}
$$

for any $x \in\left(a_{k}(s)+\delta_{\log \log }^{\varepsilon}, a_{k+1}(s)-\delta_{\log \log }^{\varepsilon}\right)$ and any $s \in I^{\varepsilon}\left(s_{0}\right)$, where we have set $d(x, s):=$ $\operatorname{dist}\left(x,\left\{a_{k}^{\varepsilon}(s), a_{k+1}^{\varepsilon}(s)\right\}\right)$ and where $C>0$ depends only on $V$ and $M_{0}$. Moreover, we also have

$$
\left\{\begin{aligned}
-\operatorname{sign}\left(\dagger_{k}\right) \mathfrak{W}_{\varepsilon}\left(a_{k}^{\varepsilon}(s)+\delta_{\log \log }^{\varepsilon}\right) & \geq \frac{1}{C}\left(\delta_{\log \log }^{\varepsilon}\right)^{-\frac{1}{\theta-1}} \\
\operatorname{sign}\left(\dagger_{k+1}\right) \mathfrak{W}_{\varepsilon}\left(a_{k+1}^{\varepsilon}(s)-\delta_{\log \log }^{\varepsilon}\right) & \geq \frac{1}{C}\left(\delta_{\log \log }^{\varepsilon}\right)^{-\frac{1}{\theta-1}} .
\end{aligned}\right.
$$

We describe next on a formal level how to obtain the desired asymptotics for $\varepsilon^{-\omega} \xi_{\varepsilon}$, as $\varepsilon \rightarrow 0$, near the point $a_{k+\frac{1}{2}}(s)$. Going back to the limiting points $\left\{a_{k}(s)\right\}_{k \in J}$ defined in Proposition 5 , we consider the subset of $\mathbb{R} \times \mathbb{R}^{+}$

$$
\mathcal{V}_{k}\left(s_{0}\right)=\bigcup_{s \in I^{*}\left(s_{0}\right)}\left(a_{k}(s), a_{k+1}(s)\right) \times\{s\}
$$

It follows from the uniform bounds established in Lemma 2, that, passing possibly to a further subsequence, we may assume that

$$
\mathfrak{W}_{\varepsilon_{n}} \rightarrow \mathfrak{W}_{*} \text { in } L_{\text {loc }}^{p}\left(\mathcal{V}_{k}\left(s_{0}\right)\right) \text {, for any } 1 \leq p<\infty .
$$


On the other hand, thanks to estimate (46), for a given point $(x, s) \in \mathcal{V}_{k}\left(s_{0}\right)$ we expand $(\mathrm{PGL})_{\varepsilon}$ near $(x, s)$ as

$$
\varepsilon^{\omega} \frac{\partial \mathfrak{W}_{\varepsilon}}{\partial s}-\frac{\partial^{2} \mathfrak{W}_{\varepsilon}}{\partial x^{2}}+2 \theta \lambda_{i\left(k+\frac{1}{2}\right)} \mathfrak{W}_{\varepsilon}^{2 \theta-1}=O\left(\varepsilon^{\frac{1}{\theta-1}}\right)
$$

Passing to the limit $\varepsilon_{n} \rightarrow 0$, we expect that for every $s \in I^{*}\left(s_{0}\right), \mathfrak{W}_{*}$ solves

$$
\left\{\begin{array}{c}
-\frac{\partial^{2} \mathfrak{W}_{*}}{\partial x^{2}}(s, \cdot)+2 \theta \lambda_{i\left(k+\frac{1}{2}\right)} \mathfrak{W}_{*}^{2 \theta-1}(s, \cdot)=0 \text { on }\left(a_{k}(s), a_{k+1}(s)\right), \\
\mathfrak{W}_{*}\left(a_{k}(s)\right)=-\operatorname{sign}\left(\dagger_{k}\right) \infty \text { and } \mathfrak{W}_{*}\left(a_{k+1}(s)\right)=\operatorname{sign}\left(\dagger_{k}\right) \infty,
\end{array}\right.
$$

the boundary conditions being a consequence of the asymptotics (47). It turns out, in view of Lemma A.1 of the Appendix, that the boundary value problem (50) has a unique solution. By scaling, and setting $r_{k}(s)=\frac{1}{2}\left(a_{k+1}(s)-a_{k}(s)\right)$, we obtain

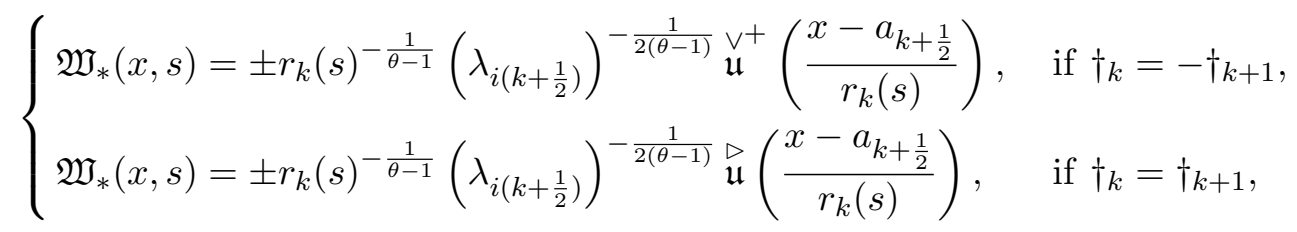

where $\mathfrak{u}^{+}$(resp. $\stackrel{\grave{u}}{)}$ ) are the unique solutions to the problems

$$
\left\{\begin{array}{l}
-\mathcal{U}_{x x}+2 \theta \mathcal{U}^{2 \theta-1}=0 \quad \text { on }(-1,+1) \\
\mathcal{U}(-1)=+\infty(\operatorname{resp} \cdot \mathcal{U}(-1)=-\infty) \text { and } \mathcal{U}(+1)=+\infty
\end{array}\right.
$$

Still on a formal level, we deduce therefore the corresponding values of the disprecancy

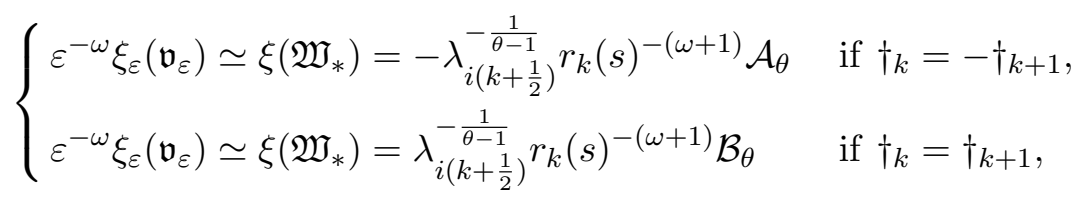

where the numbers $\mathcal{A}_{\theta}$ and $\mathcal{B}_{\theta}$ are positive, depend only on $\theta$, and correspond to the absolute value of the discrepancy of $\mathfrak{u}$ and $\mathfrak{u}$ respectively. Notice that the signs in (52) are different, the first case yields attractive forces whereas the second yields repulsive ones. Inserting this relation in (42) and arguing as for (44), we will derive the motion law.

The previous formal discussion can be put on a sound mathematical ground, relying on comparison principles and the construction of appropriate upper and lower solutions (see Section 5). This leads to the central result of this paper:

Proposition 6. Assume that conditions $\left(\mathrm{H}_{0}\right)$ and $\left(\mathrm{H}_{1}\right)$ are fulfilled. Let $0<S<S_{\max }$ be given and set

$$
\mathrm{L}_{0}:=3 \max \left\{\left|a_{k}^{0}\right|, 1 \leq k \leq \ell_{0} ;\left(\frac{S}{\rho_{0}}\right)^{\frac{1}{\omega+2}}\right\} .
$$

Assume that $\operatorname{WPI}_{\varepsilon}^{\mathrm{L}_{0}}\left(\alpha_{1} \varepsilon, 0\right)$ holds as well as $(33)$ at time $s=0$. Then $J(s)=\left\{1, \cdots, \ell_{0}\right\}$ and the functions $a_{k}^{\varepsilon}(\cdot)$ are well defined and converge uniformly on any compact interval of $(0, S)$ to the solution $a_{k}(\cdot)$ of $(\mathcal{S})$ supplemented with the initial condition $a_{k}(0)=a_{k}^{0}$. 
Notice that the combination of assumptions $\operatorname{WPI}_{\varepsilon}^{\mathrm{L}}\left(\alpha_{1} \varepsilon, 0\right),\left(\mathrm{H}_{1}\right)$ and (33) at $s=0$ implies the multiplicity one condition $\left(\mathrm{H}_{\min }\right)$. Whereas the conclusion of Proposition 6 is similar to the one of Theorem 2, the assumptions of Proposition 6 are more restrictive. Indeed, on one hand we assume the well-preparedness condition $\mathrm{WPI}_{\varepsilon}^{\mathrm{L}}$, and on the other hand we impose (33) which is far more constraining than $\left(\mathrm{H}_{\min }\right)$ : it excludes in particular the possibility of having small pairs of fronts and anti-fronts. Our next efforts are hence devoted to handle this type of situation: Proposition 6, through rescaling arguments, will nevertheless be the main building block for that task.

In order to prove Theorem 2, 3 and 4 we need to relax the assumptions on the initial data, in particular we need to analyze the behavior of data with small pairs of fronts and anti-fronts, and show that they are going to annihilate on a short interval of time. For that purpose we will consider the following situation, corresponding to confinement of the front set at initial time. Assume that for a collection of points $\left\{b_{q}^{\varepsilon}\right\}_{q \in J_{0}}$ in $\mathbb{R}$ we have

$$
\mathfrak{D}_{\varepsilon}(0) \cap \mathrm{I}_{5 \mathrm{~L}} \subset \underset{q \in J_{0}}{\cup}\left[b_{q}^{\varepsilon}-r, b_{q}^{\varepsilon}+r\right] \subset \mathrm{I}_{\mathrm{\kappa}_{0} \mathrm{~L}} \quad \text { and } \quad b_{p}^{\varepsilon}-b_{q}^{\varepsilon} \geq 3 R \quad \text { for } p \neq q \in J_{0},
$$

for some $\kappa_{0} \leq \frac{1}{2}$ and $\alpha_{1} \varepsilon \leq r \leq R / 2 \leq \mathrm{L} / 4$. It follows from (7) that if $0 \leq s \leq \rho_{0}(R-r)^{\omega+2}$ then

$$
\mathfrak{D}_{\varepsilon}(s) \cap \mathrm{I}_{4 \mathrm{~L}} \subset \underset{k \in J_{0}}{\cup}\left(b_{k}^{\varepsilon}-R, b_{k}^{\varepsilon}+R\right) \subset \mathrm{I}_{2 \kappa_{0} \mathrm{~L}} \text {, where the union is disjoint. }
$$

Consider next $0 \leq s \leq \rho_{0}(R-r)^{\omega+2}$ such that $\mathcal{W} \mathcal{P}_{\varepsilon}^{\mathrm{L}}\left(\alpha_{1} \varepsilon, s\right)$ holds, so that the front points $\left\{a_{k}^{\varepsilon}(s)\right\}_{k \in J(s)}$ are well-defined. For $q \in J_{0}$, consider $J_{q}(s)=\left\{k \in J(s), a_{k}^{\varepsilon}(s) \in\left(b_{q}^{\varepsilon}-R, b_{q}^{\varepsilon}+\right.\right.$ $R)\}$, set $\ell_{q}=\sharp J_{q}$, and write $J_{q}(s)=\left\{k_{q}, k_{q+1}, \cdots, k_{q+\ell_{q}-1}\right\}$, where $k_{1}=1$, and $k_{q}=$ $\ell_{1}+\cdots+\ell_{q-1}+1$, for $q \geq 2$. Our next result shows that, after a small time, only the repulsive forces survive at the scale given by $r$, provided the different lengths are sufficiently distinct.

Proposition 7. There exists positive constants $\alpha_{*}$ and $\rho_{*}$, depending only on $V$ and $M_{0}$, such that if (53) holds and

$$
\kappa_{0}^{-1} \geq \alpha_{*}, \quad r \geq \alpha_{*} \varepsilon\left(\frac{\mathrm{L}}{\varepsilon}\right)^{\frac{2}{\omega+2}}, \quad R \geq \alpha_{*} r,
$$

then at time

$$
s_{r}=\rho_{*} r^{\omega+2}
$$

condition $\mathcal{W P}_{\varepsilon}^{\mathrm{L}}\left(\alpha_{1} \varepsilon, s_{r}\right)$ holds and, for any $q \in J_{0}$ and any $k, k^{\prime} \in J_{q}\left(s_{r}\right)$ we have $\dagger_{k}\left(s_{r}\right)=$ $\dagger_{k}^{\prime}\left(s_{r}\right)$ or equivalently for any $k \in J_{q}\left(s_{r}\right) \backslash\left\{k_{q}\left(s_{r}\right)+\ell_{q}\left(s_{r}\right)-1\right\}$, we have

$$
\epsilon_{k+\frac{1}{2}}\left(s_{r}\right)=\dagger_{k}\left(s_{r}\right) \dagger_{k+1}\left(s_{r}\right)=+1 .
$$

Moreover, we have

$$
d_{\min }^{\varepsilon, \mathrm{L}}\left(s_{r}\right) \geq r
$$

and if $\sharp J_{q}\left(s_{r}\right) \leq 1$ for every $q \in J_{0}$, then we actually have $d_{\min }^{\varepsilon, \mathrm{L}}\left(s_{r}\right) \geq R$.

The proofs of Theorems 2, 3 and 4 are then deduced from Propositions 6 and 7 .

The paper is organized as follows. We describe in Section 2 some properties of stationary fronts, as well as for solutions to some perturbations of the stationary equations. In Section 3 
we describe several properties related to the well-preparedness assumption $\mathcal{W P}_{\varepsilon}^{\mathrm{L}}$, in particular the quantization of the energy, how it relates to dissipation, and its numerous implications for the dynamics. We provide in particular the proofs to Proposition 3, Proposition 4 and Corollary 2. In Section 4, we prove the compactness results stated in Proposition 5 and Corollary 3. Section 5, provides an expansion of the discrepancy term off the front set, from a technical point of view it is the place where the analysis differs most from the non-degenerate case. Based on this analysis, we show in Section 6 how the motion law follows from prepared datas establishing the proof to Proposition 6. In Section 7 we analyze the clearing-out of small pairs of front-antifront and more generally we present the proof of Proposition 7. Finally, in section 8 we present the proofs of the main theorems, namely Theorem 2, 3 and 4 . Several results concerning the first or second order differential equations involved in the analysis of this paper are given in separate appendices, in particular the proof of Proposition 1.

\section{Remarks on stationary solutions}

\subsection{Stationary solutions on $\mathbb{R}$ with vanishing discrepancy}

Stationary solutions are described using the method of separation of variable. For $u$ solution to (21), we multiply (21) by $u$ and verify that $\xi$ is constant. We restrict ourselves to solutions with vanishing discrepancy

$$
\xi=\frac{1}{2} \dot{u}^{2}-V(u)=0,
$$

and solve equation (2.1) by separation of variables. Let $\gamma_{i}$ be defined on $\left(\sigma_{i}, \sigma_{i+1}\right)$ by

$$
\gamma_{i}(u)=\int_{z_{i}}^{u} \frac{d s}{\sqrt{2 V(s)}}, \text { for } u \in\left(\sigma_{i}, \sigma_{i+1}\right),
$$

where we recall that $z_{i}$ is a fixed maximum point of $V$ in the interval $\left(\sigma_{i}, \sigma_{i+1}\right)$. The map $\gamma_{i}$ is one-to-one from $\left(\sigma_{i}, \sigma_{i+1}\right)$ to $\mathbb{R}$, so that we may define its inverse map $\zeta_{i}^{+}: \mathbb{R} \rightarrow\left(\sigma_{i}, \sigma_{i+1}\right)$ by

$$
\zeta_{i}^{+}(x)=\gamma_{i}^{-1}(x) \text { as well as } \zeta_{i}^{-}(x)=\gamma_{i}^{-1}(-x) \text { for } x \in \mathbb{R} .
$$

In view of the definition $(2.3)$, we have $\zeta_{i}^{ \pm}(0)=z_{i},{\zeta_{i}^{+}}^{\prime}(0)=\sqrt{2 V\left(z_{i}\right)}>0$, whereas a change of variable shows that $\zeta_{i}$ has finite energy given by the formula (8). We verify that $\zeta_{i}^{+}(\dot{\bar{\varepsilon}})$ and $\zeta_{i}^{-}\left(\frac{\dot{\varepsilon}}{\varepsilon}\right)$ solve $(2.1)$ and hence $(21)$. The next elementary result then directly follows from uniqueness in ode's:

Lemma 2.1. Let $u$ be a solution to (21) such that (2.1) holds, and such that $u\left(x_{0}\right) \in$ $\left(\sigma_{i}, \sigma_{i+1}\right)$, for some $x_{0} \in \mathbb{R}$, and some $i \in 1, \cdots \mathfrak{q}-1$. Then, there exists $a \in \mathbb{R}$ such that $u(x)=\zeta_{i}^{+}(x-a)$ or $u(x)=\zeta_{i}^{-}(x-a), \forall x \in \mathbb{R}$.

We provide a few simple properties of the functions $\zeta_{i}^{ \pm}$which enter directly in our arguments. We expand $V$ near $\sigma_{i}$ for $u \geq \sigma_{i}$ as

$$
\sqrt{V(u)}=\sqrt{\lambda_{i}}\left(u-\sigma_{i}\right)^{\theta}\left(1+O\left(u-\sigma_{i}\right)\right), \quad \text { as } u \rightarrow \sigma_{i} .
$$

Integrating, we are led to the expansion

$$
\gamma_{i}(u)=-\frac{\theta-1}{\sqrt{2 \lambda_{i}}}\left(u-\sigma_{i}\right)^{-\theta+1}\left(1+O\left(u-\sigma_{i}\right)\right), \quad \text { as } u \rightarrow \sigma_{i}
$$


and therefore also to the expansions

$$
\zeta_{i}^{ \pm}(x)=\sigma_{i}+\left(\frac{\sqrt{2 \lambda_{i}}|x|}{\theta-1}\right)^{-\frac{1}{\theta-1}}(1+o(1)), \quad \text { as } x \rightarrow \mp \infty .
$$

Similarly,

$$
\zeta_{i}^{ \pm}(x)=\sigma_{i+1}-\left(\frac{\sqrt{2 \lambda_{i+1}}|x|}{\theta-1}\right)^{-\frac{1}{\theta-1}}(1+o(1)), \quad \text { as } x \rightarrow \pm \infty,
$$

and corresponding asymptotics for the derivatives can be derived as well (e.g. using the fact that the discrepancy is zero).

For $0<\varepsilon<1$ given, and $i=1, \cdots, q-1$, consider the scaled function $\zeta_{i, \varepsilon}^{ \pm}=\zeta_{i}^{ \pm}\left(\frac{\cdot}{\varepsilon}\right)$ which is a solution to

$$
-u_{x x}+\varepsilon^{-2} V^{\prime}(u)=0
$$

hence a stationary solution to $(\mathrm{PGL})_{\varepsilon}$. Straightforward computations based on the previous expansions show that

$$
\left\{\begin{array}{l}
e_{\varepsilon}\left(\zeta_{i, \varepsilon}^{ \pm}\right)(x)=\left(2 \lambda_{i}\right)^{-\frac{1}{\theta-1}}(\theta-1)^{\frac{2 \theta}{\theta-1}} \frac{1}{\varepsilon}\left|\frac{x}{\varepsilon}\right|^{-(\omega+1)}+\underset{\frac{x}{\varepsilon} \rightarrow \mp \infty}{o}\left(\frac{1}{\varepsilon}\left|\frac{x}{\varepsilon}\right|^{-(\omega+1)}\right) \\
e_{\varepsilon}\left(\zeta_{i, \varepsilon}^{ \pm}\right)(x)=\left(2 \lambda_{i+1}\right)^{-\frac{1}{\theta-1}}(\theta-1)^{\frac{2 \theta}{\theta-1}} \frac{1}{\varepsilon}\left|\frac{x}{\varepsilon}\right|^{-(\omega+1)}+\underset{\frac{x}{\varepsilon} \rightarrow \pm \infty}{o}\left(\frac{1}{\varepsilon}\left|\frac{x}{\varepsilon}\right|^{-(\omega+1)}\right)
\end{array}\right.
$$

with $\omega$ defined in (5). Hence there is some constant $C>0$ independent of $r$ and $\varepsilon$ such that

$$
\mathfrak{S}_{i} \geq \int_{-r}^{r} e_{\varepsilon}\left(\zeta_{i, \varepsilon}^{ \pm}\right) d x \geq \mathfrak{S}_{i}-C\left(\frac{\varepsilon}{r}\right)^{\omega}
$$

\subsection{On the energy of chains of stationary solutions}

If $u$ satisfies condition $\mathrm{WPI}_{\varepsilon}^{\mathrm{L}}(\delta)$ and $\left(\mathrm{H}_{0}\right)$, we set

$$
\mathfrak{E}_{\varepsilon}^{\mathrm{L}}(u)=\sum_{k \in J} \mathfrak{S}_{i(k)} \text { and } \mathcal{E}_{\varepsilon}^{\mathrm{L}}(u)=\int_{\mathrm{I}_{2 \mathrm{~L}}} e_{\varepsilon}(u(x)) d x .
$$

Proposition 2.1. We have

$$
\begin{cases}\mathcal{E}_{\varepsilon}^{\mathrm{L}}(u) \geq \mathfrak{E}_{\varepsilon}^{\mathrm{L}}(u)-\mathrm{C}_{\mathrm{f}} M_{0}\left(\frac{\varepsilon}{\delta}\right)^{\omega} & \text { if } \mathrm{WPI}_{\varepsilon}^{\mathrm{L}}(\delta) \text { holds } \\ \mathcal{E}_{\varepsilon}^{\mathrm{L}}(u) \leq \mathfrak{E}_{\varepsilon}^{\mathrm{L}}(u)+\left(\mathrm{C}_{\mathrm{w}}+\mathrm{C}_{\mathrm{f}}\right) M_{0}\left(\frac{\varepsilon}{\delta}\right)^{\omega} & \text { if } \mathcal{W P}_{\varepsilon}^{\mathrm{L}}(\delta) \text { holds }\end{cases}
$$

Moreover, for any smooth function $\chi$ with compact support in $\mathrm{I}_{2 \mathrm{~L}}$ we have

$$
\left|\mathcal{I}_{\varepsilon}(\chi)-\sum_{k \in J} \chi\left(a_{k}\right) \mathfrak{S}_{i(k)}\right| \leq\left(\mathrm{C}_{\mathrm{w}}+\mathrm{C}_{\mathrm{f}}\right) M_{0}\left(\left(\frac{\varepsilon}{\delta}\right)^{\omega}\|\chi\|_{\infty}+\varepsilon\left\|\chi^{\prime}\right\|_{\infty}\right), \quad \text { if } \mathcal{W} \mathcal{P}_{\varepsilon}^{\mathrm{L}}(\delta) \text { holds }
$$

where $\mathcal{I}_{\varepsilon}(\chi)=\int_{\mathrm{I}_{2 \mathrm{~L}}} e_{\varepsilon}(u) \chi(x) d x$. The constant $\mathrm{C}_{\mathrm{f}}$ which appears in (2.7) and (2.8) only depends on $V$, and the constant $\mathrm{C}_{\mathrm{w}}$ appears in the definition of condition $\mathcal{W P}_{\varepsilon}^{\mathrm{L}}$. 
Proof. We estimate the integral of $\left|e_{\varepsilon}(u)-e_{\varepsilon}\left(\zeta_{i(k)}^{\dagger k}\left(\cdot-a_{k}\right)\right)\right|$ on $I_{k}$ as

$$
\frac{\varepsilon}{2} \int_{I_{k}}\left|\dot{u}^{2}-\left(\dot{\zeta}_{i(k), \varepsilon}^{\dagger_{k}}\left(\cdot-a_{k}\right)\right)^{2}\right| d x \leq \varepsilon\left\|\dot{u}-\dot{\zeta}_{i(k), \varepsilon}^{\dagger_{k}}\left(\cdot-a_{k}\right)\right\|_{L^{\infty}\left(I_{k}\right)}\left[\mathcal{E}_{\varepsilon}(u)^{\frac{1}{2}}+\mathcal{E}_{\varepsilon}\left(\zeta_{i(k), \varepsilon}^{\dagger_{k}}\right)^{\frac{1}{2}}\right] \sqrt{\frac{\delta}{\varepsilon}}
$$

and likewise we obtain

$$
\varepsilon^{-1} \int_{I_{k}}\left|V(u)-V\left(\zeta_{i(k), \varepsilon}^{\dagger_{k}}\left(\cdot-a_{k}\right)\right)\right| \leq C \frac{\delta}{\varepsilon}\left\|u-\zeta_{i(k), \varepsilon}^{\dagger_{k}}\left(\cdot-a_{k}\right)\right\|_{L^{\infty}\left(I_{k}\right)} .
$$

It suffices then to invoke $\mathrm{WPI}_{\varepsilon}^{\mathrm{L}}(\delta)$ and $\mathrm{WPO}_{\varepsilon}^{\mathrm{L}}(\delta)$ as well as the decay estimates (2.5) to derive (2.7), using the fact that since $\delta \geq \alpha_{1} \varepsilon$, negative exponentials are readily controlled by negative powers. Estimate (2.8) is derived in a very similar way, the error in $\varepsilon\left\|\chi^{\prime}\right\|_{\infty}$ being a consequence of the approximation of $\int \chi e_{\varepsilon}\left(\zeta_{i(k), \varepsilon}^{\dagger}\left(\cdot-a_{k}\right)\right)$ by $\chi\left(a_{k}\right) \mathfrak{S}_{i(k)}$.

This result shows that, if $\delta$ is sufficiently large, the energy is close to a set of discrete values, namely the finite sums of $\mathfrak{S}_{k}$. We will therefore refer to this property as the quantization of the energy, it will play an important role later when we will obtain estimates on the dissipation rate of energy.

\subsection{Study of the perturbed stationary equation}

Consider a function $u$ defined on $\mathbb{R}$ satisfying the perturbed differential equation

$$
u_{x x}=\varepsilon^{-2} V^{\prime}(u)+f,
$$

where $f \in L^{2}(\mathbb{R})$, and the energy bound $\left(\mathrm{H}_{0}\right)$. We already know, thanks to Lemma 2.1 that if $f=0$ then $u$ is of the form $\zeta_{i, \varepsilon}^{ \pm}(\cdot-a)$. Our results below, summarized here in loose terms, show that if $f$ is sufficiently small on some sufficiently large interval, then $u$ is close to a chain of translations of the functions $\zeta_{i, \varepsilon}^{ \pm}$suitably glued together on that interval.

Following the approach of [4], we first recast equation (2.9) as a system of two differential equations of first order. For that purpose, we set $w=\varepsilon u_{x}$ so that (2.9) is equivalent to the system

$$
u_{x}=\frac{1}{\varepsilon} w \text { and } w_{x}=\frac{1}{\varepsilon} V^{\prime}(u)+\varepsilon f
$$

which we may write in a more condensed form as

$$
U_{x}=\frac{1}{\varepsilon} G(U)+\varepsilon F \text { on } \mathbb{R},
$$

where we have set $U(x)=(u(x), w(x))$ and $F(x)=(0, f(x))$, and where $G$ denotes the vector field $G(u, w)=\left(w, V^{\prime}(u)\right)$. Notice that the energy bound $\left(\mathrm{H}_{0}\right)$ and assumption $\left(A_{3}\right)$ together imply a global $L^{\infty}$ bound on $u$. In turn, this $L^{\infty}$ bound imply a Lipschitz bound, denoted $C_{0}$, for the nonlinearity $G(u, w)$.

Lemma 2.2. Let $u_{1}$ and $u_{2}$ satisfy (2.9) with forcing terms $f_{1}$ and $f_{2}$, and assume that both satisfy the energy bound $\left(H_{0}\right)$. Denote by $U_{1}, U_{2}, F_{1}, F_{2}$ the corresponding solutions and forcing terms of (2.10). Then, for any $x, x_{0}$ in some arbitrary interval $I$,

$$
\left|\left(U_{1}-U_{2}\right)(x)\right| \leq\left(\left|\left(U_{1}-U_{2}\right)\left(x_{0}\right)\right|+\frac{\varepsilon^{\frac{3}{2}}}{\sqrt{2 C_{0}}}\left\|F_{1}-F_{2}\right\|_{L^{2}(I)}\right) \exp \left(\frac{C_{0}\left|x-x_{0}\right|}{\varepsilon}\right) .
$$


Proof. Since $\left(U_{1}-U_{2}\right)_{x}=G\left(U_{1}\right)-G\left(U_{2}\right)+\varepsilon\left(F_{1}-F_{2}\right)$ we obtain the inequality

$$
\left|\left(U_{1}-U_{2}\right)_{x}\right| \leq \frac{C_{0}}{\varepsilon}\left|U_{1}-U_{2}\right|+\varepsilon\left|F_{1}-F_{2}\right| .
$$

It follows from Gronwall's inequality that

$$
\left|\left(U_{1}-U_{2}\right)(x)\right| \leq \exp \left(\frac{C_{0}\left|x-x_{0}\right|}{\varepsilon}\right)\left|\left(U_{1}-U_{2}\right)\left(x_{0}\right)\right|+\left|\int_{x_{0}}^{x} \varepsilon\right|\left(F_{1}-F_{2}\right)(y)\left|\exp \left(\frac{C_{0}\left|y-x_{0}\right|}{\varepsilon}\right) d y\right| .
$$

Claim (2.11) then follows from the Cauchy-Schwarz inequality.

We will combine the previous lemma with

Lemma 2.3. Let $u$ be a solution of (2.9) satisfying $\left(H_{0}\right)$. Then

$$
\sup _{x, y \in I}\left|\xi_{\varepsilon}(u)(x)-\xi_{\varepsilon}(u)(y)\right| \leq \sqrt{2 M_{0}} \varepsilon^{\frac{1}{2}}\|f\|_{L^{2}(I)},
$$

where $I \subset \mathbb{R}$ is an arbitrary interval.

Proof. This is a direct consequence of the equality $\frac{d}{d x} \xi_{\varepsilon}(u)=\varepsilon f \frac{d}{d x} u$, the Cauchy-Schwarz inequality, and the definition of the energy.

Lemma 2.4. Let $u$ be a solution of (2.9) satisfying $\left(H_{0}\right)$. Let $L>0$ and assume that

$$
\mathcal{D}(u) \cap \mathrm{I}_{2 \mathrm{~L}} \subseteq \mathrm{I}_{\mathrm{L}} .
$$

There exist a constant $0<\kappa_{\mathrm{W}}<1$, depending only on $V$, such that if

$$
M_{0} \frac{\varepsilon}{\mathrm{L}}+M_{0}^{\frac{1}{2}} \varepsilon^{\frac{3}{2}}\|f\|_{L^{2}\left(\mathrm{I}_{\frac{3}{2} \mathrm{~L}}\right)} \leq \kappa_{\mathrm{w}}
$$

then the condition $\operatorname{WPI}_{\varepsilon}^{\mathrm{L}}(\delta)$ holds where

$$
\frac{\delta}{\varepsilon}:=-\frac{2}{\rho_{\mathrm{w}}} \log \left(M_{0} \frac{\varepsilon}{\mathrm{L}}+M_{0}^{\frac{1}{2}} \varepsilon^{\frac{3}{2}}\|f\|_{L^{2}\left(\mathrm{I}_{\frac{3}{2} \mathrm{~L}}\right)}\right),
$$

and where the constant $\rho_{\mathrm{w}}$ depends only on $M_{0}$ and $V$. Moreover, $\kappa_{\mathrm{w}}$ is sufficiently small so that $2\left|\log \kappa_{\mathrm{w}}\right| / \rho_{\mathrm{w}} \geq \alpha_{1}$, where $\alpha_{1}$ was defined in (26).

Proof. If $\mathcal{D}(u) \cap \mathrm{I}_{2 \mathrm{~L}}=\emptyset$ then there is nothing to prove. If not, we first claim that there exist a point $a_{1} \in \mathrm{I}_{\mathrm{L}}$ such that $u\left(a_{1}\right)=z_{i(1)}$ for some $i(1) \in\{1, \cdots, \mathfrak{q}-1\}$. Indeed, if not, and since the endpoints of $\mathrm{I}_{2 \mathrm{~L}}$ are not in the front set, the function $u$ would have a critical point with a critical value in the complement of $\cup_{j} B\left(\sigma_{j}, \mu_{0}\right)$. At that point, the discrepancy would therefore be larger than $C / \varepsilon$ for some constant $C>0$ depending only of $V$ (through the choice of $\left.\mu_{0}\right)$. On the other hand, since $\left|\xi_{\varepsilon}\right| \leq e_{\varepsilon}$, by averaging there exist at least one point in $\mathrm{I}_{\frac{3}{2} \mathrm{~L}}$ where the discrepancy of $u$ is smaller in absolute value than $M_{0} /(3 \mathrm{~L})$. Combined with the estimate of Lemma 2.3 on the oscillation of the discrepancy, we hence derive our first claim, provided $\kappa_{\mathrm{w}}$ in (2.12) is choosen sufficiently small. Wet set $\dagger_{1}=\operatorname{sign}\left(u^{\prime}\left(a_{1}\right)\right), u_{1}=u$ and $\left.u_{2}=\zeta_{i(1), \varepsilon}^{\dagger 1}\left(\cdot-x_{1}\right)\right)$. Since

$$
V\left(u_{1}\left(a_{1}\right)\right)=V\left(u_{2}\left(a_{1}\right)\right)=V\left(z_{i(1)}\right)
$$


and since

$$
\left|\xi_{\varepsilon}\left(u_{1}\right)\left(a_{1}\right)-\xi_{\varepsilon}\left(u_{2}\right)\left(a_{1}\right)\right|=\left|\xi_{\varepsilon}\left(u_{1}\right)\left(a_{1}\right)\right| \leq M_{0} /(3 \mathrm{~L})+\sqrt{2 M_{0}} \varepsilon^{\frac{1}{2}}\|f\|_{L^{2}\left(\mathrm{I}_{\frac{3}{2} \mathrm{~L}}\right)},
$$

we obtain

$$
\left.\left|\varepsilon\left(u_{1}^{\prime}\right)^{2}\left(a_{1}\right)-\varepsilon\left(u_{2}^{\prime}\right)^{2}\left(a_{1}\right)\right| \leq M_{0} /(\mathrm{L})+2 \sqrt{2 M_{0}} \varepsilon^{\frac{1}{2}}\|f\|_{L^{2}\left(\mathrm{I}_{\frac{3}{2}} \mathrm{~L}\right.}\right)
$$

Since also

$$
\left|u_{1}^{\prime}\left(a_{1}\right)+u_{2}^{\prime}\left(a_{1}\right)\right| \geq\left|u_{2}^{\prime}\left(a_{1}\right)\right|=\left|\sqrt{\frac{2 V\left(z_{i(1)}\right)}{\varepsilon^{2}}}\right| \geq C / \varepsilon,
$$

it follows that

$$
\left|\varepsilon\left(u_{1}^{\prime}-u_{2}^{\prime}\right)\left(a_{1}\right)\right| \leq C\left(M_{0} \frac{\varepsilon}{\mathrm{L}}+\sqrt{M_{0}} \varepsilon^{\frac{3}{2}}\|f\|_{L^{2}\left(\mathrm{I}_{\frac{3}{2} \mathrm{~L}}\right)}\right),
$$

for a constant $C>0$ which depends only on $V$. We may then apply Lemma 2.2 to $u_{1}$ and $u_{2}$ with the choice $x_{0}=a_{1}$, and for which we thus have, with the notations of Lemma 2.2,

$$
\left|\left(U_{1}-U_{2}\right)\left(x_{0}\right)\right| \leq C\left(M_{0} \frac{\varepsilon}{\mathrm{L}}+\sqrt{M_{0}} \varepsilon^{\frac{3}{2}}\|f\|_{L^{2}\left(\mathrm{I}_{\frac{3}{2} \mathrm{~L}}\right)}\right) .
$$

Estimate (2.11) then yields (24) on $I_{1}=\left[a_{1}-\delta, a_{1}+\delta\right]$, for the choice of $\delta$ given by (2.13) with $\rho_{\mathrm{w}}=4\left(C_{0}+1\right)$, where $C_{0}$ depends only on $M_{0}$ and $V$ and was defined above Lemma 2.2 .

If $\mathcal{D}(u) \cap\left(\mathrm{I}_{\frac{3}{2} \mathrm{~L}} \backslash\left[a_{1}-\delta, a_{1}+\delta\right]\right)=\emptyset$, we are done, and if not we may repeat the previous construction (the boundary points of $\left[a_{1}-\delta, a_{1}+\delta\right]$ are not part of the front set), until after finitely many steps we cover the whole front set.

We turn to the outer condition ${ }^{7} \mathrm{WPO}_{\varepsilon}^{\mathrm{L}}$.

Lemma 2.5. Let $u$ be a solution of (2.9) verifying $\left(H_{0}\right)$, and assume that for some index $i \in\{1, \cdots, \mathfrak{q}\}$

$$
u(x) \in B\left(\sigma_{i}, \mu_{0}\right) \quad \forall x \in A,
$$

where $A$ is some arbitrary bounded interval. Set $R=\operatorname{length}(A)$, let $0<\rho<R$, and set $B=\left\{x \in A \mid \operatorname{dist}\left(x, A^{c}\right)>\rho\right\}$. Then we have the estimate

$$
\mathcal{E}_{\varepsilon}(u, B) \leq \mathrm{C}_{\mathrm{o}}\left(\mathcal{E}_{\varepsilon}(u, A \backslash B)^{\frac{1}{\theta}}\left(\frac{\varepsilon}{\rho}\right)^{1+\frac{1}{\theta}}+R^{\frac{3}{2}} M_{0}^{\frac{1}{2 \theta}}\left(\frac{\varepsilon}{R}\right)^{1+\frac{1}{2 \theta}}\|f\|_{L^{2}(A)}\right),
$$

where the constant $\mathrm{C}_{\mathrm{o}}$ depends only on $V$.

Proof. Let $0 \leq \chi \leq 1$ be a smooth cut-off function with compact support in $A$ and such that $\chi \equiv 1$ on $B$ and $\left|\chi^{\prime}\right| \leq 2 / \rho$ on $A$. We multiply $(2.9)$ by $\varepsilon\left(u-\sigma_{i}\right) \chi^{2}$ and integrate on $A$. This leads to

$$
\int_{A} \varepsilon u_{x}^{2} \chi^{2}+\frac{1}{\varepsilon} V^{\prime}(u)\left(u-\sigma_{i}\right) \chi^{2}=\int_{A \backslash B} 2 \varepsilon u_{x}\left(u-\sigma_{i}\right) \chi \chi^{\prime}-\int_{A} \varepsilon f\left(u-\sigma_{i}\right) \chi^{2} .
$$

\footnotetext{
${ }^{7}$ for which several adaptations have to be carried out compared to the non-degenerate case.
} 
We estimate the first term on the right-hand side above by

$$
\begin{aligned}
\left|\int_{A \backslash B} 2 \varepsilon u_{x}\left(u-\sigma_{i}\right) \chi \chi^{\prime}\right| & \leq\left(\int_{A} \varepsilon u_{x}^{2} \chi^{2}\right)^{\frac{1}{2}}\left(\int_{A \backslash B} \varepsilon^{\theta}\left(u-\sigma_{i}\right)^{2 \theta}\right)^{\frac{1}{2 \theta}}\left(\int_{A \backslash B}\left|2 \chi^{\prime}\right|^{\frac{2 \theta}{\theta-1}}\right)^{\frac{\theta-1}{2 \theta}} \\
& \leq \frac{1}{2} \int_{A} \varepsilon u_{x}^{2} \chi^{2}+\frac{1}{2} \varepsilon^{1+\frac{1}{\theta}}\left(\int_{A \backslash B} \frac{2}{\lambda_{i}} e_{\varepsilon}(u)\right)^{\frac{1}{\theta}}\left(\frac{4}{\rho}\right)^{2}(2 \rho)^{\frac{\theta-1}{\theta}} \\
& \leq \frac{1}{2} \int_{A} u_{x}^{2} \chi^{2}+16 \lambda_{i}^{-\frac{1}{\theta}}\left(\frac{\varepsilon}{\rho}\right)^{1+\frac{1}{\theta}} \mathcal{E}_{\varepsilon}(u, A \backslash B)^{\frac{1}{\theta}}
\end{aligned}
$$

where we have used $(2)$ and the fact that length $(A \backslash B)=2 \rho$. Similarly we estimate

$$
\begin{aligned}
\left|\int_{A} \varepsilon f\left(u-\sigma_{i}\right) \chi^{2}\right| & \leq \varepsilon\|f\|_{L^{2}(A)}\left(\int_{A}\left(u-\sigma_{i}\right)^{2 \theta}\right)^{\frac{1}{2 \theta}} R^{\frac{\theta-1}{2 \theta}} \\
& \leq \varepsilon^{1+\frac{1}{2 \theta}}\|f\|_{L^{2}(A)}\left(\frac{2}{\lambda_{i}}\right)^{-1} M_{0}^{\frac{1}{2 \theta}} R^{\frac{\theta-1}{2 \theta}} .
\end{aligned}
$$

Also, by (2) we have

$$
\int_{A} \frac{1}{\varepsilon} V^{\prime}(u)\left(u-\sigma_{i}\right) \chi^{2} \geq \theta \int_{B} \frac{1}{\varepsilon} V(u)
$$

Combining the previous inequalities the conclusion follows.

Combining Lemma 2.4 with Lemma 2.5 we obtain

Proposition 2.2. Let $u$ be a solution to (2.9) satisfying assumption $\left(H_{0}\right)$, and such that $\mathcal{D}(u) \cap \mathrm{I}_{3 \mathrm{~L}} \subset \mathrm{I}_{\mathrm{L}}$. There exist positive constants ${ }^{8} \mathrm{C}_{\mathrm{w}}$ and $\alpha_{1}$, depending only on $M_{0}$ and $V$, such that if $\alpha \geq \alpha_{1}$ and if

$$
\begin{aligned}
& \text { 1. } M_{0} \frac{\varepsilon}{\mathrm{L}} \leq \frac{1}{2} \exp \left(-\frac{\rho_{\mathrm{w}}}{2} \alpha\right), \\
& \text { 2. }\|f\|_{L^{2}\left(\mathrm{I}_{3 \mathrm{~L}}\right)} \leq \frac{1}{2} M_{0}^{-\frac{1}{2}} \varepsilon^{-\frac{3}{2}} \exp \left(-\frac{\rho_{\mathrm{w}}}{2} \alpha\right), \\
& \text { 3. }\|f\|_{L^{2}\left(\mathrm{I}_{3 \mathrm{~L}}\right)} \leq \frac{\mathrm{C}_{\mathrm{w}}}{2 \mathrm{C}_{\mathrm{O}}} M_{0}^{1-\frac{1}{2 \theta}}\left(\frac{\varepsilon}{\mathrm{L}}\right)^{-1-\frac{1}{2 \theta}} \mathrm{L}^{-\frac{3}{2}} \alpha^{-\omega},
\end{aligned}
$$

then $\mathcal{W P}_{\varepsilon}^{\mathrm{L}}(\alpha \varepsilon)$ holds.

Proof. Direct substitution shows that assumptions 1. and 2. imply condition (2.12), provided $\alpha_{1}$ is choosen sufficiently large, and also imply condition $\operatorname{WPI}_{\varepsilon}^{\mathrm{L}}(\delta)$ for some $\delta \geq \alpha \varepsilon$ given by (2.13). It remains to consider $\mathrm{WPO}_{\varepsilon}^{\mathrm{L}}(\alpha \varepsilon)$. We invoke Lemma 2.5 on each of the intervals $A=\left(a_{k}+\frac{1}{2} \alpha \varepsilon, a_{k+1}-\frac{1}{2} \alpha \varepsilon\right)$, taking $B=\left(a_{k}+\alpha \varepsilon, a_{k+1}-\alpha \varepsilon\right)$. In view of $\mathrm{WPI}_{\varepsilon}^{\mathrm{L}}(\alpha \varepsilon)$ and $(2.5)$, we obtain

$$
\mathcal{E}_{\varepsilon}(u, A \backslash B) \leq C \alpha^{-\omega},
$$

and therefore

$$
\mathcal{E}_{\varepsilon}(u, A \backslash B)^{\frac{1}{\theta}} \alpha^{-1-\frac{1}{\theta}} \leq C \alpha^{-\omega},
$$

\footnotetext{
${ }^{8}$ Recall that $\mathrm{C}_{\mathrm{w}}$ enters in the definition of condition $\mathcal{W} \mathcal{P}_{\varepsilon}^{\mathrm{L}}$. A parameter named $\mathrm{C}_{\mathrm{w}}$ already appears in the statement of Proposition 2.1 above: We impose that its updated value here is be larger han its original value in Proposition 2.1 (and Proposition 2.1 remains of course true with this updated value!).
} 
where $C$ depends only on $V$. Also, in view of assumption 3. we have

$$
\mathrm{C}_{\mathrm{o}} \sum_{k} R^{\frac{3}{2}} M_{0}^{\frac{1}{2 \theta}}\left(\frac{\varepsilon}{R}\right)^{1+\frac{1}{2 \theta}}\|f\|_{L^{2}(A)} \leq \mathrm{C}_{\mathrm{o}} L^{\frac{3}{2}} M_{0}^{\frac{1}{2 \theta}}\left(\frac{\varepsilon}{L}\right)^{1+\frac{1}{2 \theta}}\|f\|_{L^{2}\left(\mathrm{I}_{3 \mathrm{~L}}\right)} \leq \frac{1}{2} \mathrm{C}_{\mathrm{w}} M_{0} \alpha^{-\omega},
$$

provided $\alpha_{1}$ is sufficiently large (third requirement). It remains to estimate $e_{\varepsilon}(u)$ on the intervals $\left(-2 \mathrm{~L}, a_{1}\right)$ and $\left(a_{\ell}, 2 \mathrm{~L}\right)$. We first use Lemma 2.5 with $A=(-3 \mathrm{~L},-\mathrm{L})$ (resp. $A=$ $(\mathrm{L}, 3 \mathrm{~L})$ and $B=\left(-\frac{5}{2} \mathrm{~L},-\frac{3}{2} \mathrm{~L}\right)$ (resp. $\left.B=\left(\frac{3}{2} \mathrm{~L}, \frac{5}{2} \mathrm{~L}\right)\right)$. This yields, using the trivial bound $\mathcal{E}_{\varepsilon}(u, A \backslash B) \leq M_{0}$, the estimate

$$
\mathcal{E}_{\varepsilon}\left(u, \mathrm{I}_{\frac{5}{2} \mathrm{~L}} \backslash \mathrm{I}_{\frac{3}{2} \mathrm{~L}}\right) \leq C\left(M_{0}^{\frac{1}{\theta}}\left(\frac{\varepsilon}{\mathrm{L}}\right)^{1+\frac{1}{\theta}}+M_{0}^{\frac{1}{2 \theta}}\left(\frac{\varepsilon}{\mathrm{L}}\right)^{\frac{1}{2 \theta}}\right) \leq C \alpha^{-\omega},
$$

in view of 1 . and provided $\alpha_{1}$ is sufficiently large. We apply one last time Lemma 2.5, with $A=\left(-2 \mathrm{~L}-\frac{1}{2} \alpha \varepsilon, a_{1}-\frac{1}{2} \alpha \varepsilon\right)$ (resp. $\left.A=\left(a_{\ell}+\frac{1}{2} \alpha \varepsilon, 2 \mathrm{~L}+\frac{1}{2} \alpha \varepsilon\right)\right)$ and $B=\left(-2 \mathrm{~L}, a_{1}-\alpha \varepsilon\right)$ (resp. $\left.B=\left(a_{\ell}+\alpha \varepsilon, 2 \mathrm{~L}\right)\right)$. Since $A \backslash B \subset \mathrm{I}_{\frac{5}{2} \mathrm{~L}} \backslash \mathrm{I}_{\frac{3}{2} \mathrm{~L}}$, it follows from (2.14) and Lemma 2.5, combined with our previous estimates, that condition $\mathrm{WPO}_{\varepsilon}^{\mathrm{L}}(\alpha \varepsilon)$ is satisfied provided we choose $\mathrm{C}_{\mathrm{w}}$ sufficiently large.

Remark 2.1. Notice that condition 1. in Proposition 2.2 is always satisfied when $\alpha \varepsilon \leq$ $\delta_{\log }^{\varepsilon}$, since $L / \varepsilon \geq 1$. Also, for $\alpha=\delta_{\log }^{\varepsilon} / \varepsilon$, assumption 3. in Proposition 2.2 is weaker than assumption 2. We therefore deduce

Corollary 2.1. Let $u$ be a solution to (2.9) satisfying assumption $\left(H_{0}\right)$, and such that $\mathcal{D}(u) \cap$ $\mathrm{I}_{3 \mathrm{~L}} \subset \mathrm{I}_{\mathrm{L}}$. If

$$
\varepsilon\|f\|_{L^{2}\left(\mathrm{I}_{3 \mathrm{~L}}\right)} \leq\left(\frac{M_{0}}{\mathrm{~L}}\right)^{\frac{1}{2}},
$$

then $\mathcal{W} \mathcal{P}_{\varepsilon}^{\mathrm{L}}\left(\delta_{\log }^{\varepsilon}\right)$ holds.

\section{Regularized fronts}

In the whole section, we assume that $v_{\varepsilon}$ is a solution of $(\mathrm{PGL})_{\varepsilon}$ which satisfies $\left(\mathrm{H}_{0}\right)$ and the confinement condition $\mathcal{C}_{\mathrm{L}, S}$.

\subsection{Finding regularized fronts}

We provide here the proof to Proposition 3, which is deduced from the following:

Lemma 3.1. Given any $s_{1}<s_{2}$ in $[0, S]$, there exist at least one time $s$ in $\left[s_{1}, s_{2}\right]$ for which $\mathfrak{v}_{\varepsilon}(\cdot, s)$ solves $(2.9)$ with

$$
\|f\|_{L^{2}\left(\mathrm{I}_{3 \mathrm{~L}}\right)}^{2} \equiv \varepsilon^{\omega-1}\left\|\partial_{s} \mathfrak{v}_{\varepsilon}(\cdot, s)\right\|_{L^{2}\left(\mathrm{I}_{3 \mathrm{~L}}\right)}^{2} \leq \varepsilon^{\omega-1} \frac{\operatorname{dissip}_{\varepsilon}^{3 \mathrm{~L}}\left(s_{1}, s_{2}\right)}{s_{2}-s_{1}} \leq \varepsilon^{\omega-1} \frac{M_{0}}{s_{2}-s_{1}} .
$$

Proof. It is a direct mean value argument, taking into account the rescaling of $(\mathrm{PGL})_{\varepsilon}$ according to our rescaling of time. 
Proof of Proposition 3. We invoke Lemma 3.1, and from (3.1) and the assumption $s_{2}-s_{1}=$ $\varepsilon^{\omega+1} \mathrm{~L}$ of Proposition 3, we derive exactly the assumption (2.15) in Corollary 2.1, from which the conclusion follows.

Following the same argument, but relying on Lemma 2.4 and Proposition 2.2 rather than on Corollary 2.1, we readily obtain

Proposition 3.1. For $\alpha_{1} \leq \alpha \leq \delta_{\log }^{\varepsilon}$ :

1. Each subinterval of $[0, S]$ of size $\mathfrak{q}_{0}(\alpha) \varepsilon^{\omega+2}$ contains at least one time $s$ at which $\mathrm{WPI}_{\varepsilon}^{\mathrm{L}}(\alpha \varepsilon, s)$ holds, where

$$
\mathfrak{q}_{0}(\alpha)=4 M_{0}^{2} \exp \left(\rho_{\mathrm{w}} \alpha\right) .
$$

2. Each subinterval of $[0, S]$ of size $\mathfrak{q}_{0}(\alpha, \beta) \varepsilon^{\omega+2}$ contains at least one time $s$ at which $\mathcal{W P}_{\varepsilon}^{\mathrm{L}}(\alpha \varepsilon, s)$ holds, where

$$
\beta:=\frac{\mathrm{L}}{\varepsilon} \quad \text { and } \quad \mathfrak{q}_{0}(\alpha, \beta)=\max \left(\mathfrak{q}_{0}(\alpha),\left(\frac{2 \mathrm{C}_{\mathrm{o}}}{\mathrm{C}_{\mathrm{w}}}\right)^{2}\left(\frac{\beta}{M_{0}}\right)^{1-\frac{1}{\theta}} \alpha^{2 \omega}\right) .
$$

\subsection{Local dissipation}

For $s \in[0, S]$, set $\mathcal{E}_{\varepsilon}^{\mathrm{L}}(s)=\mathcal{E}_{\varepsilon}^{\mathrm{L}}\left(\mathfrak{v}_{\varepsilon}(s)\right)$ and, when $\operatorname{WPI}_{\varepsilon}^{\mathrm{L}}\left(\alpha_{1} \varepsilon, s\right)$ holds, $\mathfrak{E}_{\varepsilon}^{\mathrm{L}}(s)=\mathfrak{E}_{\varepsilon}^{\mathrm{L}}\left(\mathfrak{v}_{\varepsilon}(s)\right), \mathfrak{E}_{\varepsilon}^{\mathrm{L}}$ being defined in (2.6). We assume throughout that $s_{1} \leq s_{2}$ are contained in $[0, S]$, and in some places (in view of (28) that $s_{2} \geq \mathrm{L}^{2} \varepsilon^{\omega}$.

Proposition 3.2. If $s_{2} \geq \mathrm{L}^{2} \varepsilon^{\omega}$, we have

$$
\mathcal{E}_{\varepsilon}^{\mathrm{L}}\left(s_{2}\right)+\operatorname{dissip}_{\varepsilon}^{\mathrm{L}}\left(s_{1}, s_{2}\right) \leq \mathcal{E}_{\varepsilon}^{\mathrm{L}}\left(s_{1}\right)+100 \mathrm{C}_{e} \mathrm{~L}^{-(\omega+2)}\left(s_{2}-s_{1}\right)+\mathrm{C}_{\mathrm{e}}\left(1+M_{0}\right)\left(\frac{\mathrm{L}}{\varepsilon}\right)^{-\omega} .
$$

Proof. Let $0 \leq \varphi \leq 1$ be a smooth function with compact support in $\mathrm{I}_{2 \mathrm{~L}}$, such that $\varphi(x)=1$ on $\mathrm{I}_{\frac{5}{3} \mathrm{~L}},\left|\varphi^{\prime \prime}\right| \leq 100 \mathrm{~L}^{-2}$. It follows from the properties of $\varphi$ and (28) that

$$
\mathcal{I}_{\varepsilon}(s, \varphi) \leq \mathcal{E}_{\varepsilon}^{\mathrm{L}}(s) \text { for } s \in\left(s_{1}, s_{2}\right) \text { and } \mathcal{I}_{\varepsilon}\left(s_{2}, \varphi\right) \geq \mathcal{E}_{\varepsilon}^{\mathrm{L}}\left(s_{2}\right)-\mathrm{C}_{\mathrm{e}}\left(\frac{\mathrm{L}}{\varepsilon}\right)^{-\omega},
$$

which combined with (36) yields

$$
\mathcal{E}_{\varepsilon}^{\mathrm{L}}\left(s_{2}\right)+\operatorname{dissip}_{\varepsilon}^{\mathrm{L}}\left(s_{1}, s_{2}\right) \leq \mathcal{E}_{\varepsilon}^{\mathrm{L}}\left(s_{1}\right)+\mathrm{C}_{\mathrm{e}}\left(\frac{\mathrm{L}}{\varepsilon}\right)^{-\omega}+\varepsilon^{-\omega} \int_{s_{1}}^{s_{2}} \mathcal{F}_{S}\left(s, \varphi, \mathfrak{v}_{\varepsilon}\right) d s
$$

where $\mathcal{F}_{S}$ is defined in (37). The estimate (3.4) is then obtained invoking the inequality $\left|\xi_{\varepsilon}\right| \leq e_{\varepsilon}$ to bound the term involving $\mathcal{F}_{S}$ : combined with (28) for times $s \geq \mathrm{L}^{2} \varepsilon^{\omega}$ and with assumption $\left(H_{0}\right)$ for times $s \leq \mathrm{L}^{2} \varepsilon^{\omega}$.

If $\mathcal{W} \mathcal{P}_{\varepsilon}^{\mathrm{L}}\left(\delta, s_{1}\right)$ and $\operatorname{WPI}_{\varepsilon}^{\mathrm{L}}\left(\delta^{\prime}, s_{2}\right)$ hold, for some $\delta, \delta^{\prime} \geq \alpha_{1} \varepsilon$ and $s_{2} \geq \mathrm{L}^{2} \varepsilon^{\omega}$, then combining inequality (3.4) with the first inequality (2.7) applied to $\mathfrak{v}_{\varepsilon}\left(s_{2}\right)$ as well as the second applied 
to $\mathfrak{v}_{\varepsilon}\left(s_{1}\right)$ we obtain

$$
\begin{aligned}
& \mathfrak{E}_{\varepsilon}^{\mathrm{L}}\left(s_{2}\right)+\operatorname{dissip}_{\varepsilon}^{\mathrm{L}}\left(s_{1}, s_{2}\right) \\
& \leq \mathcal{E}_{\varepsilon}^{\mathrm{L}}\left(s_{2}\right)+\mathrm{C}_{\mathrm{f}} M_{0}\left(\frac{\varepsilon}{\delta^{\prime}}\right)^{\omega}+\operatorname{dissip}_{\varepsilon}^{\mathrm{L}}\left(s_{1}, s_{2}\right) \\
& \leq \mathcal{E}_{\varepsilon}^{\mathrm{L}}\left(s_{1}\right)+100 \mathrm{C}_{e} \mathrm{~L}^{-(\omega+2)}\left(s_{2}-s_{1}\right)+\mathrm{C}_{\mathrm{f}} M_{0}\left(\frac{\varepsilon}{\delta^{\prime}}\right)^{\omega}+\mathrm{C}_{\mathrm{e}}\left(1+M_{0}\right)\left(\frac{\varepsilon}{\mathrm{L}}\right)^{\omega} \\
& \leq \mathfrak{E}_{\varepsilon}^{\mathrm{L}}\left(s_{1}\right)+\left(\mathrm{C}_{\mathrm{w}}+\mathrm{C}_{\mathrm{f}}\right) M_{0}\left(\frac{\varepsilon}{\delta}\right)^{\omega}+\mathrm{C}_{\mathrm{f}} M_{0}\left(\frac{\varepsilon}{\delta^{\prime}}\right)^{\omega}+100 \mathrm{C}_{e} \mathrm{~L}^{-(\omega+2)}\left(s_{2}-s_{1}\right)+\mathrm{C}_{\mathrm{e}}\left(1+M_{0}\right)\left(\frac{\varepsilon}{\mathrm{L}}\right)^{\omega} .
\end{aligned}
$$

We deduce from this inequality an estimate for the dissipation between $s_{1}$ and $s_{2}$ and an upper bound on $\mathcal{E}_{\varepsilon}^{\mathrm{L}}\left(s_{2}\right)$ :

Corollary 3.1. Assume that $\mathcal{W P}_{\varepsilon}^{\mathrm{L}}\left(\delta, s_{1}\right)$ and $\operatorname{WPI}_{\varepsilon}^{\mathrm{L}}\left(\delta^{\prime}, s_{2}\right)$ hold, for some $\delta, \delta^{\prime} \geq \alpha_{1} \varepsilon$ and $s_{2} \geq \mathrm{L}^{2} \varepsilon^{\omega}$, and that $\mathfrak{E}_{\varepsilon}^{\mathrm{L}}\left(s_{1}\right)=\mathfrak{E}_{\varepsilon}^{\mathrm{L}}\left(s_{2}\right)$. Then

$$
\begin{gathered}
\operatorname{dissip}_{\varepsilon}^{\mathrm{L}}\left[s_{1}, s_{2}\right] \leq\left(\mathrm{C}_{\mathrm{w}}+\mathrm{C}_{\mathrm{f}}\right) M_{0}\left(\frac{\varepsilon}{\delta}\right)^{\omega}+\mathrm{C}_{\mathrm{f}} M_{0}\left(\frac{\varepsilon}{\delta^{\prime}}\right)^{\omega}+100 \mathrm{C}_{e} \mathrm{~L}^{-(\omega+2)}\left(s_{2}-s_{1}\right)+\mathrm{C}_{\mathrm{e}}\left(1+M_{0}\right)\left(\frac{\varepsilon}{\mathrm{L}}\right)^{\omega}, \\
\mathcal{E}_{\varepsilon}^{\mathrm{L}}\left(s_{2}\right)-\mathfrak{E}_{\varepsilon}^{\mathrm{L}}\left(s_{2}\right) \leq\left(\mathrm{C}_{\mathrm{w}}+\mathrm{C}_{\mathrm{f}}\right) M_{0}\left(\frac{\varepsilon}{\delta}\right)^{\omega}+100 \mathrm{C}_{e} \mathrm{~L}^{-(\omega+2)}\left(s_{2}-s_{1}\right)+\mathrm{C}_{\mathrm{e}}\left(1+M_{0}\right)\left(\frac{\varepsilon}{\mathrm{L}}\right)^{\omega} .
\end{gathered}
$$

\subsection{Quantization of the energy}

Let $s \in[0, S]$ and $\delta \geq \alpha_{1} \varepsilon$, and assume that $\mathfrak{v}_{\varepsilon}$ satisfies $\mathcal{W} \mathcal{P}_{\varepsilon}^{\mathrm{L}}(\delta, s)$. The front energy $\mathfrak{E}_{\varepsilon}^{\mathrm{L}}(s)$, by definition, may only take a finite number of values, and is hence quantized. We emphasize that, at this stage, $\mathfrak{E}_{\varepsilon}^{\mathrm{L}}(s)$ is only defined assuming condition $\operatorname{WPI}_{\varepsilon}^{\mathrm{L}}(\delta, s)$ holds. However, the value of $\mathfrak{E}_{\varepsilon}^{\mathrm{L}}(s)$ does not depend on $\delta$, provided that $\delta \geq \alpha_{1} \varepsilon$, so that it suffices ultimately to check that condition $\operatorname{WPI}_{\varepsilon}^{\mathrm{L}}\left(\alpha_{1} \varepsilon, s\right)$ is fulfilled.

Since $\mathfrak{E}_{\varepsilon}(s)$ may take only a finite number of values, let $\mu_{1}>0$ be the smallest possible difference between two distinct such values. Let $\mathrm{L}_{0} \equiv \mathrm{L}_{0}\left(s_{1}, s_{2}\right)>0$ be such that

$$
100 \mathrm{C}_{e} \mathrm{~L}_{0}^{-(\omega+2)}\left(s_{2}-s_{1}\right)=\frac{\mu_{1}}{4}
$$

and finally choose $\alpha_{1}$ sufficiently large so that

$$
\left(\left(2 \mathrm{C}_{\mathrm{f}}+\mathrm{C}_{\mathrm{w}}\right) M_{0}+\mathrm{C}_{e}\left(1+M_{0}\right)\right) \alpha_{1}^{-\omega} \leq \frac{\mu_{1}}{4} .
$$

As a direct consequence of (3.5), (3.6), (3.7) and the definition of $\mu_{1}$ we obtain

Corollary 3.2. For $s_{1} \leq s_{2} \in[0, S]$ with $s_{2} \geq \varepsilon^{\omega} L^{2}$, assume that $\mathcal{W P}_{\varepsilon}^{\mathrm{L}}\left(\alpha_{1} \varepsilon, s_{1}\right)$ and $\operatorname{WPI}_{\varepsilon}^{\mathrm{L}}\left(\alpha_{1} \varepsilon, s_{2}\right)$ hold and that $\mathrm{L} \geq \mathrm{L}_{0}\left(s_{1}, s_{2}\right)$. Then we have $\mathfrak{E}_{\varepsilon}^{\mathrm{L}}\left(s_{2}\right) \leq \mathfrak{E}_{\varepsilon}^{\mathrm{L}}\left(s_{1}\right)$. Moreover, if $\mathfrak{E}_{\varepsilon}^{\mathrm{L}}\left(s_{2}\right)<\mathfrak{E}_{\varepsilon}^{\mathrm{L}}\left(s_{1}\right)$, then $\mathfrak{E}_{\varepsilon}^{\mathrm{L}}\left(s_{2}\right)+\mu_{1} \leq \mathfrak{E}_{\varepsilon}^{\mathrm{L}}\left(s_{1}\right)$.

In the opposite direction we have:

Lemma 3.2. For $s_{1} \leq s_{2} \in[0, S]$, assume that $\mathrm{WPI}_{\varepsilon}^{\mathrm{L}}\left(\alpha_{1} \varepsilon, s_{1}\right)$ and $\mathrm{WPI}_{\varepsilon}^{\mathrm{L}}\left(\alpha_{1} \varepsilon, s_{2}\right)$ hold and that $\mathrm{L} \geq \mathrm{L}_{0}\left(s_{1}, s_{2}\right)$. Assume also that

$$
s_{2}-s_{1} \leq \rho_{0}\left(\frac{1}{8} d_{\min }^{\varepsilon, \mathrm{L}}\left(s_{1}\right)\right)^{\omega+2} .
$$


Then we have $\mathfrak{E}_{\varepsilon}^{\mathrm{L}}\left(s_{2}\right) \geq \mathfrak{E}_{\varepsilon}^{\mathrm{L}}\left(s_{1}\right)$. In case of equality, we have $J\left(s_{1}\right)=J\left(s_{2}\right)$ and

$$
\sigma_{i\left(k \pm \frac{1}{2}\right)}\left(s_{1}\right)=\sigma_{i\left(k \pm \frac{1}{2}\right)}\left(s_{1}\right), \text { for any } k \in J\left(s_{1}\right) \text { and } d_{\min }^{\varepsilon, \mathrm{L}}\left(s_{2}\right) \geq \frac{1}{2} d_{\min }^{\varepsilon, \mathrm{L}}\left(s_{1}\right) .
$$

Proof. It a consequence of the bound (7) in Theorem 1 on the speed of the front set combined with assumption (3.8). Indeed, this implies that for arbitrary $s \in\left[s_{1}, s_{2}\right]$, the front set a time $s$ is contained in a neighborhood of size $d_{\min }^{\varepsilon, \mathrm{L}}\left(s_{1}\right) / 8$ of the front set at time $s_{1}$. In view of the definition of $d_{\min }^{\varepsilon, \mathrm{L}}\left(s_{1}\right)$, and of the continuity in time of the solution, this implies that for all $k_{0} \in J\left(s_{1}\right)$ the set

$$
\mathcal{A}_{k_{0}}=\left\{k \in J\left(s_{2}\right) \text { such that } a_{k}^{\varepsilon}\left(s_{2}\right) \in\left[a_{k_{0}}^{\varepsilon}\left(s_{1}\right)-\frac{1}{4} d_{\min }^{\varepsilon, \mathrm{L}}\left(s_{1}\right), a_{k_{0}}^{\varepsilon}\left(s_{1}\right)+\frac{1}{4} d_{\min }^{\varepsilon, \mathrm{L}}\left(s_{1}\right)\right]\right\}
$$

is non empty, since it must contain a front connecting $\sigma_{i\left(k_{0}-\frac{1}{2}\right)}\left(s_{1}\right)$ to $\sigma_{i\left(k_{0}+\frac{1}{2}\right)}\left(s_{1}\right)$. In particular, summing over all fronts in $\mathcal{A}_{k_{0}}$, we obtain

$$
\sum_{k \in \mathcal{A}_{k_{0}}} \mathfrak{S}_{i(k)}^{\mathrm{L}} \geq \mathfrak{S}_{i\left(k_{0}\right)}^{\mathrm{L}}
$$

with equality if and only if $\sharp \mathcal{A}_{k_{0}}=1$. Summing over all indices $k_{0}$, we are led to the conclusion.

\subsection{Propagating regularized fronts}

We discuss in this subsection the case of equality $\mathfrak{E}_{\varepsilon}^{\mathrm{L}}\left(s_{1}\right)=\mathfrak{E}_{\varepsilon}^{\mathrm{L}}\left(s_{2}\right)$. We assume throughout that we are given $\delta_{\log }^{\varepsilon} \geq \delta>\alpha_{1} \varepsilon$ and two times $s_{1} \leq s_{2} \in\left[\varepsilon^{\omega} \mathrm{L}^{2}, S\right]$ such that

$\mathcal{C}\left(\delta, L, s_{1}, s_{2}\right)$

$$
\begin{cases}\mathcal{W} \mathcal{P}_{\varepsilon}^{\mathrm{L}}\left(\delta, s_{1}\right) \quad \text { and } & \operatorname{WPI}_{\varepsilon}^{\mathrm{L}}\left(\delta, s_{2}\right) \quad \text { hold } \\ \mathfrak{E}_{\varepsilon}^{\mathrm{L}}\left(s_{1}\right)=\mathfrak{E}_{\varepsilon}^{\mathrm{L}}\left(s_{2}\right), & \text { with } \mathrm{L} \geq \mathrm{L}_{0}\left(s_{1}, s_{2}\right) .\end{cases}
$$

Under that assumption, our first result shows that $\mathfrak{v}_{\varepsilon}$ remains well-prepared on almost the whole time interval $\left[s_{1}, s_{2}\right]$, with a smaller $\delta$ though.

Proposition 3.3. There exists $\alpha_{2} \geq \alpha_{1}$, depending only on $V, M_{0}$ and $\mathrm{C}_{\mathrm{w}}$, with the following property. Assume that $\mathcal{C}\left(\delta, L, s_{1}, s_{2}\right)$ holds with $\alpha_{2} \varepsilon \leq \delta \leq \delta_{\log }^{\varepsilon}$, then property $\mathcal{W} \mathcal{P}_{\varepsilon}^{\mathrm{L}}\left(\Lambda_{\log }(\delta), s\right)$ holds for any time $s \in\left[s_{1}+\varepsilon^{2+\omega}, s_{2}\right]$, where

$$
\Lambda_{\log }(\delta)=\frac{\omega}{\rho_{\mathrm{w}}} \varepsilon\left(\log \frac{\delta}{\varepsilon}\right) .
$$

The proof of Proposition 3.3 relies on the following.

Lemma 3.3. Assume that $\mathcal{C}\left(\delta, L, s_{1}, s_{2}\right)$ holds with $\delta \geq \alpha_{1} \varepsilon$. We have the estimate, for $s \in\left[s_{1}+\varepsilon^{\omega+2}, s_{2}\right]$

$$
\int_{\mathrm{I}_{\frac{3}{2} \mathrm{~L}}}\left|\partial_{t} v_{\varepsilon}\left(x, s \varepsilon^{-\omega}\right)\right|^{2} d x \leq C \varepsilon^{-3} \operatorname{dissip}_{\varepsilon}^{\mathrm{L}}\left[s, s-\varepsilon^{\omega+2}\right] .
$$


Proof of Lemma 3.3. Differentiating equation $\left(\mathrm{PGL}_{\varepsilon}\right)$ with respect to time, we are led to

$$
\left|\partial_{t}\left(\partial_{t} v_{\varepsilon}\right)-\partial_{x x}\left(\partial_{t} v_{\varepsilon}\right)\right| \leq \frac{C}{\varepsilon^{2}}\left|\partial_{t} v_{\varepsilon}\right|
$$

It follows from standard parabolic estimates, working for $x \in \mathrm{I}_{2 \mathrm{~L}}$ on the cylinder $\Lambda_{\varepsilon}(x)=$ $[x-\varepsilon, x+\varepsilon] \times\left[t-\varepsilon^{2}, t\right]$, where $t:=s \varepsilon^{-\omega}$, that for any point $y \in\left[x-\frac{\varepsilon}{2}, x+\frac{\varepsilon}{2}\right]$ we have

$$
\left|\partial_{t} v_{\varepsilon}(y, t)\right| \leq C \varepsilon^{-\frac{3}{2}}\left\|\partial_{t} v_{\varepsilon}\right\|_{L^{2}\left(\Lambda_{\varepsilon}(x)\right)} .
$$

Taking the square of the previous inequality, and integrating over $\left[x-\frac{\varepsilon}{2}, x+\frac{\varepsilon}{2}\right]$, we are led to

$$
\int_{x-\frac{\varepsilon}{2}}^{x+\frac{\varepsilon}{2}}\left|\partial_{t} v_{\varepsilon}(y, t)\right|^{2} d y \leq C \varepsilon^{-2} \int_{[x-2 \varepsilon, x+2 \varepsilon] \times\left[t-\varepsilon^{2}, t\right]}\left|\partial_{t} v_{\varepsilon}(y, t)\right|^{2} d y .
$$

A elementary covering argument then yields

$$
\int_{\mathrm{I}_{\frac{3}{2} \mathrm{~L}}}\left|\partial_{t} v_{\varepsilon}(y, t)\right|^{2} d y \leq C \varepsilon^{-2}\left\|\partial_{t} v_{\varepsilon}\right\|_{L^{2}\left(\mathrm{I}_{\frac{5}{3} \mathrm{~L}} \times\left[t-\varepsilon^{2}, t\right]\right)}^{2} \leq C \varepsilon^{-3} \operatorname{dissip}_{\varepsilon}^{\mathrm{L}}\left[s, s-\varepsilon^{\omega+2}\right] .
$$

Proof of Proposition 3.3. In view of Proposition 3.1, of Corollary 3.2, and of assumption $\mathcal{C}\left(\delta, \mathrm{L}, s_{1}, s_{2}\right)$, we may assume, without loss of generality, that

$$
s_{2}-s_{1} \leq 2 \mathfrak{q}_{0}(\delta / \varepsilon, \mathrm{L} / \varepsilon)
$$

Let $s \in\left(s_{1}+\varepsilon^{\omega+2}, s_{2}\right)$, and consider once more the map $u=\mathfrak{v}_{\varepsilon}(\cdot, s)$, so that $u$ is a solution to $(2.9)$, with source term $f=\partial_{t} v_{\varepsilon}\left(\cdot, s \varepsilon^{-\omega}\right)$. It follows from Lemma 3.3, combined with the first of Corrolary 3.1 on the dissipation, that

$$
\|f\|_{L^{2}\left(\mathrm{I}_{\frac{3}{2} \mathrm{~L}}\right)}^{2} \leq C \varepsilon^{-3}\left[\left(\mathrm{C}_{\mathrm{w}}+2 \mathrm{C}_{\mathrm{f}}\right) M_{0}\left(\frac{\varepsilon}{\delta}\right)^{\omega}+100 \mathrm{C}_{e} \mathrm{~L}^{-(\omega+2)}\left(s_{2}-s_{1}\right)+\mathrm{C}_{\mathrm{e}}\left(1+M_{0}\right)\left(\frac{\varepsilon}{\mathrm{L}}\right)^{\omega}\right] .
$$

Notice that (3.11) combined with the assumption $\delta \leq \delta_{\log }^{\varepsilon}$ yields

$$
100 \mathrm{C}_{e} \mathrm{~L}^{-(\omega+2)}\left(s_{2}-s_{1}\right) \leq C\left(\frac{\varepsilon}{\delta}\right)^{\omega} .
$$

We deduce from Lemma 2.4, imposing on $\alpha_{2}$ the additional condition $\frac{\omega}{\rho_{\mathrm{w}}}\left(\log \alpha_{2}\right) \geq \alpha_{1}$, that $\mathrm{WPI}_{\varepsilon}^{\mathrm{L}}\left(\left(\Lambda_{\log }(\delta), s\right)\right.$ holds. It remains to show that $\mathrm{WPO}_{\varepsilon}^{\mathrm{L}}\left(\Lambda_{\log }(\delta), s\right)$ holds likewise. To that aim, we invoke (3.1) which we use with the choice $s_{1}=s_{1}$ and $s_{2}=s$. This yields, taking once more (3.11) into account,

$$
\mathfrak{E}_{\varepsilon}^{\mathrm{L}}(s)-\mathcal{E}_{\varepsilon}^{\mathrm{L}}(s) \leq\left(C+\mathrm{C}_{\mathrm{w}}\right)\left(\frac{\varepsilon}{\delta}\right)^{\omega}
$$

Combining this relation with (2.5) and the first inequality of (2.7), we deduce that

$$
\int_{\Omega} e_{\varepsilon}\left(\mathfrak{v}_{\varepsilon}(s)\right) d s \leq\left(C+\mathrm{C}_{\mathrm{w}}\right)\left(\frac{\varepsilon}{\delta}\right)^{\omega}+C\left(\frac{\varepsilon}{\Lambda_{\log }(\delta)}\right)^{\omega} \leq \mathrm{C}_{\mathrm{w}} M_{0}\left(\frac{\varepsilon}{\Lambda_{\log }(\delta)}\right)^{\omega}
$$

provided $\alpha_{2}$ is choosen sufficiently large. 
In view of (3.8) and (7), we introduce the function

$$
\mathfrak{q}_{1}(\alpha):=\left(\frac{\mathfrak{q}_{0}(\alpha)}{\rho_{0}}\right)^{\frac{1}{\omega+2}}
$$

which represents therefore the maximum displacement of the front set in the interval of time needed (at most) to find two consecutive times at which $\mathrm{WPI}_{\varepsilon}^{\mathrm{L}}(\alpha \varepsilon)$ holds.

From Proposition 3.3 and Lemma 3.2 we deduce

Corollary 3.3. Let $s \in\left[\varepsilon^{\omega} \mathrm{L}^{2}, S\right]$ and $\alpha_{2} \leq \alpha \leq \delta_{\log }^{\varepsilon}$, and assume that $\mathcal{W} \mathcal{P}_{\varepsilon}^{\mathrm{L}}(\alpha \varepsilon, s)$ holds as well as $d_{\min }^{\varepsilon, \mathrm{L}}(s) \geq 16 \mathfrak{q}_{1}(\alpha) \varepsilon$. Then $\mathcal{W P}_{\varepsilon}^{\mathrm{L}}\left(\Lambda_{\log }(\alpha \varepsilon), s^{\prime}\right)$ holds for any $s+\varepsilon^{2+\omega} \leq s^{\prime} \leq \mathcal{T}_{0}^{\varepsilon}(\alpha, s)$, where

$$
\mathcal{T}_{0}^{\varepsilon}(\alpha, s)=\max \left\{s+\varepsilon^{2+\omega} \leq s^{\prime} \leq S \quad \text { s.t. } \quad d_{\text {min }}^{\varepsilon, \mathrm{L}}\left(s^{\prime \prime}\right) \geq 8 \mathfrak{q}_{1}(\alpha) \varepsilon \quad \forall s^{\prime \prime} \in\left[s+\varepsilon^{\omega+2}, s^{\prime}\right]\right\} .
$$

We complete this section presenting the

Proof of Proposition 4. This follows directly from Corollary 3.3 with the choice $\alpha=\delta_{\log }^{\varepsilon}$, noticing that $\Lambda_{\log }\left(\delta_{\log }^{\varepsilon}\right)=\delta_{\log \log }^{\varepsilon}$.

Proof of Corollary 2. If we assume moreover that $s_{0} \geq \varepsilon^{\omega} \mathrm{L}^{2}$ and that $\mathcal{W} \mathcal{P}_{\varepsilon}^{\mathrm{L}}\left(\delta_{\log }^{\varepsilon}, s_{0}\right)$ holds, then it is a direct consequence of the inclusion (7) and Proposition 4, taking into account the assumption (33). If we assume only that $s_{0} \geq 0$ and that $\mathcal{W} \mathcal{P}_{\varepsilon}^{\mathrm{L}}\left(\alpha_{1} \varepsilon, s_{0}\right)$ holds, then it suffices to consider the first time $s_{0}^{\prime} \geq s_{0}+\varepsilon^{\omega} \mathrm{L}^{2}$ at which $\mathcal{W} \mathcal{P}_{\varepsilon}^{\mathrm{L}}\left(\delta_{\log }^{\varepsilon}, s_{0}^{\prime}\right)$ holds and to rely on Proposition 4 likewise. Indeed, since $s_{0}^{\prime}-s_{0} \leq \varepsilon^{\omega} \mathrm{L}^{2}+\varepsilon^{\omega+1} \mathrm{~L}$ by Proposition 3 , we may apply Corollary 3.2 and Lemma 3.2 for $s_{1}=s_{0}$ and $s_{2}=s_{0}^{\prime}$, which yields $\mathfrak{E}_{\varepsilon}^{\mathrm{L}}\left(s_{0}\right)=\mathfrak{E}_{\varepsilon}^{\mathrm{L}}\left(s_{0}^{\prime}\right)$ and therefore also the same asymptotics for $d_{\min }^{\S, \mathrm{L}}$ at times $s_{0}$ and $s_{0}^{\prime}$.

\section{A first compactness results for the front points}

The purpose of this section is to provide the proofs of Proposition 5 and Corollary 3.

Proof of Proposition 5. As mentioned, we choose the test functions (indepently of time) so that they are affine near the front points for any $s \in I^{\varepsilon}\left(s_{0}\right)$. More precisely, for a given $k_{0} \in J$ we impose the following conditions on the test functions $\chi \equiv \chi_{k_{0}}$ in (42):

$$
\left\{\begin{array}{l}
\chi \text { has compact support in }\left[a_{k}^{\varepsilon}\left(s_{0}\right)-\frac{1}{3} d_{\min }^{*}\left(s_{0}\right), a_{k}^{\varepsilon}\left(s_{0}\right)+\frac{1}{3} d_{\min }^{*}\left(s_{0}\right)\right], \\
\chi \text { is affine on the interval }\left[a_{k}^{\varepsilon}\left(s_{0}\right)-\frac{1}{4} d_{\min }^{*}\left(s_{0}\right), a_{k}^{\varepsilon}\left(s_{0}\right)+\frac{1}{4} d_{\min }^{*}\left(s_{0}\right)\right], \text { with } \chi^{\prime}=1 \text { there } \\
\|\chi\|_{L^{\infty}(\mathbb{R})} \leq C d_{\min }^{*}\left(s_{0}\right),\left\|\chi^{\prime}\right\|_{L^{\infty}(\mathbb{R})} \leq C \text { and }\left\|\chi^{\prime \prime}\right\|_{L^{\infty}(\mathbb{R})} \leq C d_{\min }^{*}\left(s_{0}\right)^{-1} .
\end{array}\right.
$$

It follows from Corollary 2 that, for $\varepsilon$ sufficiently small, we are in position to claim (42) and (43) for arbitrary $s_{1}$ and $s_{2}$ in the full interval $I^{*}\left(s_{0}\right)$. Combined with the first estimate of Corollary 3.1, with $\delta=\delta^{\prime}=\delta_{\log \log }^{\varepsilon}$, this yields the conclusion (44).

Proof of Corollary 3. The family of functions $\left(\mathfrak{v}_{\varepsilon}\right)_{0<\varepsilon<1}$ is equi-continuous on every compact subset of the interval $I^{*}(s)$, so that the conclusion follows from the Arzela-Ascoli theorem. 


\section{$5 \quad$ Refined asymptotics off the front set}

\subsection{Relaxations towards stationary solutions}

Throughout this section, we assume that we are in the situation described by Corollary 2, in particular $\mathrm{L}$ is fixed and $\varepsilon$ will tend to zero. Our main purpose is then to provide rigorous mathematical statements and proofs concerning the properties of the function $\mathfrak{W}_{\varepsilon_{n}}=\mathfrak{W}_{\varepsilon_{n}}^{k}$ defined in (45), for given $k \in J$, which have been presented, most of them in a formal way, in Subsection 1.5. We notice first that we may expand $V^{\prime}$ near $\sigma \equiv \sigma_{i\left(k+\frac{1}{2}\right)}$ as

$$
V^{\prime}(\sigma+u)=2 \theta \lambda u^{2 \theta-1}(1+u g(u)),
$$

where $g$ is a some smooth function on $\mathbb{R}$ and where we have set for the sake of simplicity $\lambda=\lambda_{i\left(k+\frac{1}{2}\right)}$. We work on the sets $\mathcal{V}_{k}\left(s_{0}\right)$ defined in (48) and on their analogs at the $\varepsilon$ level

$$
\mathcal{V}_{k}^{\varepsilon}\left(s_{0}\right)=\underset{s \in I^{\varepsilon}\left(s_{0}\right)}{\cup} \mathcal{J}_{\varepsilon}(s) \times\{s\} \equiv \underset{s \in I^{\varepsilon}\left(s_{0}\right)}{\cup}\left(a_{k}^{\varepsilon}(s)+\delta_{\log \log }^{\varepsilon}, a_{k+1}^{\varepsilon}(s)-\delta_{\log \log }^{\varepsilon}\right) \times\{s\} .
$$

We will therefore work only with arbitrary small values of $u$. Let $u_{0}>0$ be sufficiently small so that $|u g(u)| \leq 1 / 4$ on $\left(-u_{0}, u_{0}\right)$ and $V^{\prime}(\sigma+u)$ is strictly increasing on $\left(-u_{0}, u_{0}\right)$, convex on $\left(0, u_{0}\right)$ and concave on $\left(-u_{0}, 0\right)$. For small values of $\varepsilon$, the value of $u$ in (5.1), in view of (46) in Lemma 2 , will not exceed $u_{0}$, and we may therefore assume for the considerations in this section that $u g(u)=u_{0} g\left(u_{0}\right)$, if $u \geq u_{0}$ and $-u g(u)=u_{0} g\left(u_{0}\right)$, if $u \leq-u_{0}$. Equation $(\mathrm{PGL})_{\varepsilon}$ translates into the following equation for $\mathfrak{W}_{\varepsilon}$

$$
L_{\varepsilon}\left(\mathfrak{W}_{\varepsilon}\right) \equiv \varepsilon^{\omega} \frac{\partial \mathfrak{W}_{\varepsilon}}{\partial s}-\frac{\partial^{2} \mathfrak{W}_{\varepsilon}}{\partial x^{2}}+\lambda f_{\varepsilon}\left(\mathfrak{W}_{\varepsilon}\right)=0
$$

where we have set

$$
f_{\varepsilon}(w)=2 \theta w^{2 \theta-1}\left(1+\varepsilon^{\frac{1}{\theta-1}} w g\left(\varepsilon^{\frac{1}{\theta-1}} w\right)\right) .
$$

Notice that our assumption yield in particular

$$
\left|f_{\varepsilon}(w)\right| \geq \frac{3}{2} \theta|w|^{2 \theta-1} .
$$

The analysis of the parabolic equation (5.3) is the core of this section. As mentioned, our results express convergence to stationary solutions. We first provide a few properties concerning these stationary solutions: the first lemma describes stationary solutions involved in the attractive case, whereas the second lemma is used in the repulsive case.

Lemma 5.1. Let $r>0$ and $0<\varepsilon<1$. There exist unique solutions ${\mathfrak{\mathfrak { u } _ { \varepsilon , r }}}^{+}\left(\right.$resp. $\stackrel{\mathfrak{u}_{\varepsilon, r}^{-}}{\mathfrak{v}^{-}}$) to

$$
\left\{\begin{array}{l}
-\frac{d \mathcal{U}}{d x^{2}}+\lambda f_{\varepsilon}(U)=0 \text { on }(-r, r), \\
\mathcal{U}(-r)=+\infty(\operatorname{resp} \cdot \mathcal{U}(-r)=-\infty) \text { and } \mathcal{U}(r)=+\infty(\operatorname{resp} \cdot \mathcal{U}(r)=-\infty)
\end{array}\right.
$$

Moreover we have,

$$
C^{-1} r^{-\frac{1}{\theta-1}} \leq \mathfrak{\mathfrak { u }}_{\varepsilon, r}^{+} \leq C(r-|x|)^{-\frac{1}{\theta-1}} \text { and } C^{-1} r^{-\frac{1}{\theta-1}} \leq-\mathfrak{\mathfrak { u }}_{\varepsilon, r}^{-} \leq C(r-|x|)^{-\frac{1}{\theta-1}},
$$

for some constant $C>0$ depending only on $V$. 
Lemma 5.2. Let $r>0$ and $0<\varepsilon<1$ be given. There exists a unique solution $\stackrel{\triangleright}{\mathfrak{u}, r}$ to

$$
-\frac{d \mathcal{U}}{d x^{2}}+\lambda f_{\varepsilon}(U)=0 \text { on }(-r, r), \mathcal{U}(-r)=-\infty \text { and } \mathcal{U}(r)=+\infty .
$$

These and related results are standard and have been considered since the works of Keller [12] and Osserman [14] in the fifties, at least regarding existence. The convexity and concavity assumptions are sufficient for uniqueness. We refer to Lemma A.1 in the Appendix for a short discussion of the case a a pure power nonlinearity.

We set $r^{\varepsilon}(s)=r_{k+\frac{1}{2}}^{\varepsilon}(s)=\frac{1}{2}\left(a_{k+1}^{\varepsilon}(s)-a_{k}^{\varepsilon}(s)\right)$. Our aim is to provide sufficiently accurate expansions of $\mathfrak{W}_{\varepsilon}$ and the renormalized discrepancy $\varepsilon^{-\omega} \xi_{\varepsilon}$ on neighborhoods of the points $a_{k+\frac{1}{2}}^{\varepsilon}(s)$, for instance the intervals

$$
\Theta_{k+\frac{1}{2}}^{\varepsilon}(s)=a_{k+\frac{1}{2}}^{\varepsilon}(s)+\left[-\frac{7}{8} r^{\varepsilon}(s), \frac{7}{8} r^{\varepsilon}(s)\right]=\left[a_{k}^{\varepsilon}(s)+\frac{1}{8} r^{\varepsilon}(s), a_{k+1}^{\varepsilon}(s)-\frac{1}{8} r^{\varepsilon}(s)\right] .
$$

We first turn to the the attractive case $\dagger_{k}=-\dagger_{k+1}$. We may assume additionally that

$$
k \in\{1, \cdots, \ell-1\} \text { and } \dagger_{k}=-\dagger_{k+1}=1,
$$

the case $\dagger_{k}=-\dagger_{k+1}=-1$ being handled similarly.

Proposition 5.1. If (5.8) hold and $\varepsilon$ is sufficiently small, then for any $s \in I^{\varepsilon}\left(s_{0}\right)$ and every $x \in \Theta_{k+\frac{1}{2}}^{\varepsilon}(s)$ we have the estimate

$$
\left|\mathfrak{W}_{\varepsilon}(x, s)-\lambda^{-\frac{1}{2(\theta-1)}} \stackrel{\mathfrak{u}_{r^{\varepsilon}(s)}^{+}}{\mathrm{P}^{\prime}}(x)\right| \leq C \varepsilon^{\min \left(\frac{1}{\omega+2}, \frac{\omega-1}{2(\theta-1)}\right)} .
$$

The repulsive case corresponds to $\dagger_{k}=\dagger_{k+1}$ and we may assume as above that

$$
k \in\{1, \cdots, \ell-1\} \text { and } \dagger_{k}=\dagger_{k+1}=1 .
$$

Proposition 5.2. If (5.10) hold and $\varepsilon$ is sufficiently small, then for any $s \in I^{\varepsilon}\left(s_{0}\right)$ and every $x \in \Theta_{k+\frac{1}{2}}^{\varepsilon}(s)$ we have the estimate

$$
\left|\mathfrak{W}_{\varepsilon}(x, s)-\lambda^{-\frac{1}{2(\theta-1)}} \stackrel{\triangleright}{\mathfrak{u}_{r} \varepsilon}(s)(x)\right| \leq C \varepsilon^{\min \left(\frac{1}{\omega+2}, \frac{\omega-1}{2(\theta-1)}\right)} .
$$

Combining these results with parabolic estimates, we obtain estimates for the discrepancy.

Proposition 5.3. If $\varepsilon$ is sufficiently small, then for any $s \in I^{\varepsilon}\left(s_{0}\right)$ and every $x \in \Theta_{k+\frac{1}{2}}^{\varepsilon}(s)$ we have the estimate

$$
\left|\varepsilon^{-\omega} \xi_{\varepsilon}\left(\mathfrak{v}_{\varepsilon}\right)-\lambda_{i\left(k+\frac{1}{2}\right)}^{-\frac{1}{2(\theta-1)}} r^{\varepsilon}(s)^{-(\omega+1)} \gamma_{k+\frac{1}{2}}\right| \leq C \varepsilon^{\frac{1}{\theta^{2}}}
$$

where

$$
\left\{\begin{array}{l}
\gamma_{k+\frac{1}{2}}=A_{\theta} \text { if } \dagger_{k}=-\dagger_{k+1} \\
\gamma_{k+\frac{1}{2}}=B_{\theta} \text { if } \dagger_{k}=\dagger_{k+1} .
\end{array}\right.
$$

For the outer regions, corresponding to $k=0$ and $k=\ell$ estimates for the discrepancy are directly deduced from the crude estimates provided by Proposition 2. Proposition 5.3 provides a rigorous ground to the formal computation (52) of the introduction, and hence allows to derive the precise motion law. The proofs of Proposition 5.1 and Proposition 5.2 however are the central part of this section. Note that by no mean the estimates provided in Propositions 5.1, 5.2 and 5.3 are optimal; our goal was only to obtain convergence estimates, valid for all $\varepsilon$ sufficiently small, uniformly on $\cup_{s \in I^{\varepsilon}\left(s_{0}\right)} \Theta_{k+\frac{1}{2}}^{\varepsilon}(s) \times\{s\}$. 


\subsection{Preliminary results}

We first turn to the proof of Lemma 2, which provides first properties of $\mathfrak{W}_{\varepsilon}$.

Proof of Lemma 2. Let $x \in\left(a_{k}(s)+\delta_{\log \log }^{\varepsilon}, a_{k+1}(s)-\delta_{\operatorname{loglog}}^{\varepsilon}\right)$ and any $s \in I^{\varepsilon}\left(s_{0}\right)$, and recall that $d(x, s):=\operatorname{dist}\left(x,\left\{a_{k}^{\varepsilon}(s), a_{k+1}^{\varepsilon}(s)\right\}\right)$. In view of Proposition 3 , and in particular of estimate (31), it suffices to show that

$$
\mathfrak{v}_{\varepsilon}(y, s) \in B\left(\sigma_{i}, \mu_{0}\right) \quad \text { for all }(y, s) \in\left[x-\frac{d(x, s)}{2}, x+\frac{d(x, s)}{2}\right] \times\left[s-\varepsilon^{\omega} d(x, s)^{2}, s\right] .
$$

By Theorem 1, on such a time scale the front set moves at most by a distance

$$
d:=\left(\frac{\varepsilon^{\omega} d(x, s)^{2}}{\rho_{0}}\right)^{\frac{1}{\omega+2}} \leq \rho_{0}^{-\frac{1}{\omega+2}}\left(\frac{\varepsilon}{\delta_{\log \log }^{\varepsilon}}\right)^{\frac{\omega}{\omega+2}} d(x, s) \leq \frac{d(x, s)}{4},
$$

provided $\varepsilon / \mathrm{L}$ is sufficiently small. More precisely, Theorem 1 only provides one inclusion, forward in time, but its combination with Corrolary 2 provides both forward and backward inclusions (for times in the interval $I^{\varepsilon}\left(s_{0}\right)$ ), from which the conclusion then follows.

For the analysis of the scalar parabolic equation (5.3), we will extensively use the fact that the map $f_{\varepsilon}$ is non-decreasing on $\mathbb{R}$, allowing comparison principles. The desired estimates for $\mathfrak{W}_{\varepsilon}$ will be obtained using appropriate choices of sub- and super-solutions. The construction of these functions involve a number of elementary solutions. First, we use the functions $\mathcal{W}_{\varepsilon}^{ \pm}$, independent of the space variable $x$ and solving the ordinary differential equation

$$
\left\{\begin{array}{l}
\varepsilon^{\omega} \frac{\partial \mathcal{W}_{\varepsilon}^{ \pm}}{\partial s}=-\lambda f_{\varepsilon}\left(\mathcal{W}_{\varepsilon}^{ \pm}\right) \\
\mathcal{W}_{\varepsilon}(0)= \pm \infty
\end{array}\right.
$$

Using separation of variables, we may construct such a solution which verifies the bounds

$$
0<\mathcal{W}_{\varepsilon}^{+}(s) \leq C \varepsilon^{\frac{\omega}{2(\theta-1)}}[\lambda s]^{-\frac{1}{2(\theta-1)}} \text { and } 0 \geq \mathcal{W}_{\varepsilon}^{-}(s) \geq-C \varepsilon^{\frac{\omega}{2(\theta-1)}}[\lambda s]^{-\frac{1}{2(\theta-1)}},
$$

so that it relaxes quickly to zero. We will also use solutions of the standard heat equation and rely in several places on the next remark:

Lemma 5.3. Let $\Phi$ be a non negative solution to the heat equation $\varepsilon^{\omega} \partial_{s} \Phi-\Phi_{x x}=0$, and $U$ be such that $L_{\varepsilon}(U)=0$. Then $L_{\varepsilon}(U+\Phi) \geq 0$, and $L_{\varepsilon}(U-\Phi) \leq 0$.

Proof. Notice that $L_{\varepsilon}(U \pm \Phi)=\lambda\left(f_{\varepsilon}(U \pm \Phi)-f_{\varepsilon}(U)\right)$, so that the conclusion follows from the fact that $f_{\varepsilon}$ is non-decreasing.

Next, let $s$ be given $I^{\varepsilon}\left(s_{0}\right)$. By translation invariance, we may assume without loss of generality that

$$
a_{k+\frac{1}{2}}^{\varepsilon}(s)=0 .
$$

We set $h_{\varepsilon}=\left(\varepsilon / 2 \rho_{0}\right)^{\frac{1}{\omega+2}}$, and consider the cylinders

$$
\Lambda_{\varepsilon}^{\text {ext }}(s)=\mathcal{J}_{\varepsilon}^{\text {ext }}(s) \times[s-\varepsilon, s] \text { and } \Lambda_{\varepsilon}^{\text {int }}(s)=\mathcal{J}_{\varepsilon}^{\text {int }}(s) \times[s-\varepsilon, s],
$$


where $\mathcal{J}_{\varepsilon}^{\text {int }}(s)=\left[-r_{\text {int }}^{\varepsilon}(s), r_{\text {int }}^{\varepsilon}(s)\right], \mathcal{J}_{\varepsilon}^{\text {ext }}(s)=\left[-r_{\text {ext }}^{\varepsilon}(s), r_{\text {ext }}^{\varepsilon}(s)\right]$ with

$$
r_{\text {ext }}^{\varepsilon}(s)=r^{\varepsilon}(s)+2 h_{\varepsilon} \text { and } r_{\text {int }}^{\varepsilon}(s)=r^{\varepsilon}(s)-2 h_{\varepsilon} .
$$

If $\varepsilon$ is sufficiently small, in view of (7) we have the inclusions, with $\mathcal{V}_{k}^{\varepsilon}\left(s_{0}\right)$ defined in (5.2),

$$
\Lambda_{\varepsilon}^{\mathrm{int}}(s) \subset \Pi_{\varepsilon}(s) \equiv \mathcal{V}_{k}^{\varepsilon}\left(s_{0}\right) \cap([s-\varepsilon, s] \times \mathbb{R}) \subset \Lambda_{\varepsilon}^{\mathrm{ext}}(s) .
$$

As a matter of fact, still for $\varepsilon$ sufficiently small, we have for any $\tau \in[s-\varepsilon, s]$,

$$
\left\{\begin{aligned}
-r_{\text {ext }}^{\varepsilon}(s)+h_{\varepsilon} & \leq a_{k}^{\varepsilon}(\tau)+\delta_{\log \log }^{\varepsilon} \leq-r_{\text {int }}^{\varepsilon}(s)-h_{\varepsilon}, \\
r_{\text {int }}^{\varepsilon}+h_{\varepsilon} & \leq a_{k+1}^{\varepsilon}(\tau)-\delta_{\log l o g}^{\varepsilon} \leq r_{\text {ext }}^{\varepsilon}(s)-h_{\varepsilon}\left(s_{0}\right) .
\end{aligned}\right.
$$

We also consider the parabolic boundary of $\Lambda_{\varepsilon}^{\mathrm{ext}}(s)$

$$
\begin{aligned}
\partial_{p} \Lambda_{\varepsilon}^{\mathrm{ext}}(s) & =\left[-r_{\text {ext }}^{\varepsilon}(s), r_{\text {ext }}^{\varepsilon}(s)\right] \times\{s-\varepsilon\} \cup\left\{-r_{\text {ext }}^{\varepsilon}\right\} \times[s-\varepsilon, s] \cup\left\{r_{\text {ext }}^{\varepsilon}\right\} \times[s-\varepsilon, s] \\
& =\partial \Lambda_{\varepsilon}^{\text {ext }}(s) \backslash\left[-r_{\text {ext }}^{\varepsilon}(s), r_{\text {ext }}^{\varepsilon}(s)\right] \times\{s\}
\end{aligned}
$$

and define $\partial_{p} \Lambda_{\varepsilon}^{\text {int }}(s)$ accordingly. Finally, we set

$$
\partial_{p} \Pi_{\varepsilon}(s)=\partial\left(\Pi_{\varepsilon}(s)\right) \backslash\left[a_{k}^{\varepsilon}(s)+\delta_{\log \log }^{\varepsilon}, a_{k+1}^{\varepsilon}(s)-\delta_{\log \log }^{\varepsilon}\right] \times\{s\} .
$$

A first application of the comparison principle leads to the following bounds:

Proposition 5.4. For $x \in \mathcal{J}_{\varepsilon}^{\text {int }}(s)$

$$
\left\{\begin{array}{l}
\mathfrak{W}_{\varepsilon}(x, s) \leq \mathfrak{u}_{\varepsilon, r_{\text {int }}^{\varepsilon}}^{+}(x)+C \varepsilon^{\frac{\omega-1}{2(\theta-1)}} \\
\mathfrak{W}_{\varepsilon}(x, s) \geq \mathfrak{u}_{\varepsilon, r_{\text {int }}^{\varepsilon}}(x)-C \varepsilon^{\frac{\omega-1}{2(\theta-1)}}
\end{array}\right.
$$

Proof. We work on the cylinder $\Lambda_{\varepsilon}^{\text {int }}(s)$ and consider there the comparison map

$$
W_{\varepsilon}^{\text {sup }}(y, \tau)=\mathfrak{u}_{\varepsilon, r_{\text {int }}^{\varepsilon}}^{+}(y)+\mathcal{W}_{\varepsilon}(\tau-(s-\varepsilon)) \text { for }(y, \tau) \in \Lambda_{\varepsilon}^{\text {int }}(s) .
$$

Since the two functions on the r.h.s of the definition of $W_{\varepsilon}^{\text {sup }}$ are positive solutions to (5.3) and since $f_{\varepsilon}$ is superadditive on $\mathbb{R}^{+}$, that is, since

$$
f_{\varepsilon}(a+b) \geq f_{\varepsilon}(a)+f_{\varepsilon}(b) \text { provided } a \geq 0, b \geq 0,
$$

we deduce that

$$
L_{\varepsilon}\left(W_{\varepsilon}^{\sup }(y, \tau)\right) \geq 0 \text { on } \Lambda_{\varepsilon}^{\text {int }}(s) \text { with } W_{\varepsilon}^{\text {sup }}(y, \tau)=+\infty \text { for }(y, \tau) \in \partial_{p} \Lambda_{\varepsilon}^{\text {int }},
$$

so that $W^{\sup _{\varepsilon}}(x, s) \geq \mathfrak{W}_{\varepsilon}$ on $\partial_{p} \Lambda_{\varepsilon}^{\text {int }}$. It follows that $W_{\varepsilon}^{\text {sup }}(y, \tau) \geq \mathfrak{W}_{\varepsilon}$ on $\Lambda_{\varepsilon}^{\text {int }}$, which, combined with (5.15) immediately leads to the first inequality. The second is derived similarly.

At this stage, the constructions are some somewhat different in the case of attractive and repulsive forces, so that we need to distinguish the two cases. 


\subsection{The attractive case}

We assume here that $\dagger_{k}=-\dagger_{k+1}$. Without loss of generality, we may assume that

$$
\dagger_{k}=-\dagger_{k+1}=1 \text {, }
$$

the case $\dagger_{k}=-\dagger_{k}=-1$ being handled similarly. The purpose of this subsection is to provide the proof to Proposition 5.1. We split the proof into separate lemmas, the main efforts being devoted to the construction of subsolutions. We start with the following lower bound:

Lemma 5.4. Assume that (5.21) holds. Then, for $x \in \mathcal{J}_{\varepsilon}\left(s-\frac{\varepsilon}{2}\right)$, we have the lower bound

$$
\mathfrak{W}_{\varepsilon}\left(x, s-\frac{\varepsilon}{2}\right) \geq-C \varepsilon^{\frac{\omega-1}{2(\theta-1)}} .
$$

Proof. In view of (47), we notice that

$$
\mathfrak{W}_{\varepsilon}(y, \tau) \geq 0 \text { on } \partial_{p} \Pi_{\varepsilon}(s) \backslash\left[a_{k}(s-\varepsilon)+\delta_{\operatorname{loglog}}^{\varepsilon}, a_{k+1}(s-\varepsilon)-\delta_{\log \log }^{\varepsilon}\right] \times\{s-\varepsilon\} .
$$

We consider next the function $W_{\varepsilon}$ defined for $\tau \geq s-\varepsilon$ by $W_{\varepsilon}(y, \tau)=\mathcal{W}_{\varepsilon}^{-}(\tau-(s-\varepsilon))$. Since $W_{\varepsilon}<0$, and since $W_{\varepsilon}(s-\varepsilon)=-\infty$, we obtain $W_{\varepsilon} \leq \mathfrak{W}_{\varepsilon}$ on $\partial_{p} \Pi_{\varepsilon}(s)$, so that, by the comparison principle we are led to $W_{\varepsilon} \leq \mathfrak{W}_{\varepsilon}$ on $\Pi_{\varepsilon}(s)$ leading to the conclusion.

Proposition 5.5. Assume that (5.21) holds. We have the lower bound for $x \in \mathcal{J}_{\varepsilon}(s)$

$$
\mathfrak{W}_{\varepsilon}(x, s) \geq \mathfrak{u}_{\varepsilon, r_{\text {ext }}^{\varepsilon}}^{+}(x)-C \varepsilon^{-\frac{1}{3 \theta-1}} \exp \left(-\pi^{2} \frac{\varepsilon^{-\omega+1}}{32\left(r^{\varepsilon}(s)\right)^{2}}\right) .
$$

Proof. On $\mathcal{J}_{\varepsilon}\left(s-\frac{\varepsilon}{2}\right)$ we consider the map $\varphi_{\varepsilon}$ defined by

$$
\varphi_{\varepsilon}(x)=\inf \left\{\mathfrak{W}_{\varepsilon}\left(x, s-\frac{\varepsilon}{2}\right)-\mathfrak{u}_{\varepsilon, r_{\text {ext }}^{\varepsilon}}^{+}(x), 0\right\} \leq 0 .
$$

Invoking (5.18) and estimates (5.6) for $\mathfrak{u}_{\varepsilon, r_{\text {ext }}^{\varepsilon}}^{+}$, we obtain, for $x \in \mathcal{J}_{\varepsilon}\left(s-\frac{\varepsilon}{2}\right)$

$$
0 \leq \mathfrak{\mathfrak { u }}_{\varepsilon, r_{\mathrm{ext}}^{\varepsilon}}^{+}(x) \leq C h_{\varepsilon}^{-\frac{1}{\theta-1}},
$$

which combined with Lemma 5.4 yields

$$
\left|\varphi_{\varepsilon}(x)\right| \leq C h_{\varepsilon}^{-\frac{1}{\theta-1}} \text { for } x \in \mathcal{J}_{\varepsilon}\left(s-\frac{\varepsilon}{2}\right) .
$$

Combining (5.24), estimate (5.6) of Lemma 5.1 and estimate (47) of Lemma 2, we deduce that, if $\varepsilon$ is sufficiently small then

$$
\varphi_{\varepsilon}\left(a_{k}^{\varepsilon}\left(s-\frac{\varepsilon}{2}\right)+\delta_{\log \log }^{\varepsilon}\right)=\varphi_{\varepsilon}\left(a_{k+1}^{\varepsilon}\left(s-\frac{\varepsilon}{2}\right)-\delta_{\log \log }^{\varepsilon}\right)=0 .
$$

We extend $\varphi_{\varepsilon}$ by 0 outside the set $\mathcal{J}_{\varepsilon}\left(s-\frac{\varepsilon}{2}\right)$, and consider the solution $\Phi_{\varepsilon}$ to

$$
\left\{\begin{array}{l}
\varepsilon^{\omega} \frac{\partial \Phi_{\varepsilon}}{\partial \tau}-\frac{\partial \Phi_{\varepsilon}}{\partial x^{2}}=0 \text { on } \Lambda_{\varepsilon}^{\mathrm{ext}}(s) \cap\left\{\tau \geq s-\frac{\varepsilon}{2}\right\} \\
\Phi_{\varepsilon}\left(x, s-\frac{\varepsilon}{2}\right)=\varphi_{\varepsilon}(x) \quad \text { for } x \in \mathcal{J}_{\varepsilon}^{\operatorname{ext}}\left(s-\frac{\varepsilon}{2}\right) \\
\Phi_{\varepsilon}\left( \pm r_{\mathrm{ext}}^{\varepsilon}(s), \tau\right)=0 \quad \text { for } \tau \in\left(s-\frac{\varepsilon}{2}, s\right) .
\end{array}\right.
$$


Notice that $\Phi_{\varepsilon} \leq 0$. We consider next on $\Lambda_{\varepsilon}^{\text {ext }}(s) \cap\left\{\tau \geq s-\frac{\varepsilon}{2}\right\}$ the function $W_{\varepsilon}^{\text {inf }}$ defined by

$$
W_{\varepsilon}^{\inf }(y, \tau)=\mathfrak{u}_{\varepsilon, r_{\text {ext }}^{\varepsilon}}^{+}(y)+\Phi_{\varepsilon}(y, \tau)
$$

It follows from Lemma 5.3 that $L_{\varepsilon}\left(W_{\varepsilon}^{\text {inf }}\right) \leq 0$, so that $W_{\varepsilon}^{\text {inf }}$ is a subsolution. Since $W_{\varepsilon}^{\text {inf }} \leq \mathfrak{W}_{\varepsilon}$ on $\partial_{p}\left(\Pi_{\varepsilon}(s) \cap\left\{\tau \geq s-\frac{\varepsilon}{2}\right\}\right)$ it follows in particular that

$$
W_{\varepsilon}^{\text {inf }} \leq \mathfrak{W}_{\varepsilon} \text { on } \mathcal{J}_{\varepsilon}(s) .
$$

To complete the proof, we rely on the next linear estimates for $\Phi_{\varepsilon}$.

Lemma 5.5. We have the bound, for $y \in \mathcal{J}_{\varepsilon}^{\text {ext }}$ and $\tau \in\left(s-\frac{\varepsilon}{2}, s\right)$

$$
\left|\Phi_{\varepsilon}(y, \tau)\right| \leq C \exp \left(-\pi^{2} \varepsilon^{-\omega} \frac{\left(\tau-\left(s-\frac{\varepsilon}{2}\right)\right.}{16\left(r^{\varepsilon}(s)\right)^{2}}\right)\left\|\varphi_{\varepsilon}\right\|_{L^{\infty}\left(\mathcal{J}_{\varepsilon}\left(s-\frac{\varepsilon}{2}\right)\right)} .
$$

We postpone the proof of Lemma 5.5 and complete the proof of Proposition 5.5.

Proof of Proposition 5.5 completed. Combining Lemma 5.5 with (5.25), we are led, for $x \in \mathcal{J}_{\varepsilon}(s)$, to

$$
\left|\Phi_{\varepsilon}(x, s)\right| \leq C h_{\varepsilon}^{-\frac{1}{\theta-1}} \exp \left(-\pi^{2} \frac{\varepsilon^{-\omega+1}}{32\left(r^{\varepsilon}(s)\right)^{2}}\right) .
$$

The conclusion then follows, invoking (5.28).

Proof of Lemma 5.5. Consider on the interval $\left[-2 r^{\varepsilon}(s), 2 r^{\varepsilon}(s)\right]$ the function $\psi(x)$ defined by $\psi(x)=\cos \left(\frac{\pi}{4 r^{\varepsilon}(s)} x\right)$, so that $-\ddot{\psi}=\frac{\pi^{2}}{16}\left(r^{\varepsilon}(s)\right)^{-2} \psi, \psi \geq 0, \psi\left(-2 r^{\varepsilon}(s)\right)=\psi\left(2 r^{\varepsilon}(s)\right)=0$ and $\psi(x) \geq 1 / 2$ for $x \in\left[-r_{\text {ext }}^{\varepsilon}(s), r_{\text {ext }}^{\varepsilon}(s)\right]$. Hence, we obtain

$\varepsilon^{\omega} \Psi_{\tau}-\Psi_{x x}=0$ on $\Lambda_{\varepsilon}^{\operatorname{ext}}(s) \cap\left\{\tau \geq s-\frac{\varepsilon}{2}\right\}$, where $\Psi(x, \tau)=\exp \left(-\pi^{2} \varepsilon^{-\omega} \frac{\tau-\left(s-\frac{\varepsilon}{2}\right)}{16 r^{\varepsilon}(s)^{2}}\right) \psi(x)$.

On the other hand, for $(y, \tau) \in \partial_{p}\left(\Lambda_{\varepsilon}^{\operatorname{ext}}(s) \cap\left\{\tau \geq s-\frac{\varepsilon}{2}\right\}\right)$ we have

$$
\left|\Phi_{\varepsilon}(y, \tau)\right| \leq\left\|\varphi_{\varepsilon}\right\|_{L^{\infty}\left(\mathcal{J}_{\varepsilon}\left(s-\frac{\varepsilon}{2}\right)\right)} 2 \Psi(y, \tau)
$$

and the conclusion follows therefore from the comparison principle for the heat equation.

Proof of Proposition 5.1 completed. Combining the upper bound (5.19) of Proposition 5.4 with the lower bound (5.22) of Proposition 5.5, we are led, for $\varepsilon$ sufficiently small, to

$$
{\stackrel{\mathfrak{u}}{\varepsilon, r_{\text {ext }}^{\varepsilon}}}^{+}(x)-A_{\varepsilon} \leq \mathfrak{W}_{\varepsilon}(x, s) \leq \mathfrak{\mathfrak { u }}_{\varepsilon, r_{\text {int }}^{\varepsilon}}^{+}(x)+A_{\varepsilon},
$$

where we have set

$$
A_{\varepsilon}=C \varepsilon^{\frac{\omega-1}{2(\theta-1)}} .
$$

The conclusion (5.9) then follows from Proposition A.1 of the Appendix combined with the definition of $h_{\varepsilon}$ and (A.7). 


\subsection{The repulsive case}

In this subsection, we assume throughout that $\dagger_{k}=\dagger_{k+1}$ and may assume moreover that

$$
\dagger_{k}=\dagger_{k+1}=1
$$

the case $\dagger_{k}=\dagger_{k}=-1$ is handled similarly. The main purpose of this subsection is to provide the proof of Proposition 5.2, the central part being the construction of accurate supersolutions, subsolutions being provided by the same construction. We assume as before that (5.16) holds, and use as comparison map $\mathfrak{U}_{\varepsilon}$ defined on $\mathcal{I}_{\varepsilon}^{\text {trs }}(s) \equiv\left(-r_{\text {ext }}^{\varepsilon}(s), r_{\text {int }}^{\varepsilon}(s)\right)$ by

$$
\mathfrak{U}_{\varepsilon}(\cdot) \equiv \stackrel{\triangleright}{\mathfrak{u}}_{\varepsilon, r^{\varepsilon}(s)}\left(\cdot+2 h_{\varepsilon}\right),
$$

so that $\mathfrak{U}_{\varepsilon}(x) \rightarrow+\infty$ as $x \rightarrow r_{\text {int }}^{\varepsilon}(s), \mathfrak{U}_{\varepsilon}(x) \rightarrow-\infty$ as $x \rightarrow-r_{\text {ext }}^{\varepsilon}(s)$ and $\left|\mathfrak{U}_{\varepsilon}\left(-r^{\varepsilon}(s)\right)\right| \leq$ $C h_{\varepsilon}^{-\frac{1}{\theta-1}}$.

Proposition 5.6. For $x \in\left(a_{k}(s)+\delta_{\log \log }^{\varepsilon}, r_{\text {int }}^{\varepsilon}(s)\right)$ we have the inequality, where $C>0$ denotes some constant

$$
\mathfrak{W}_{\varepsilon}(x, s) \leq \mathfrak{U}_{\varepsilon}(x)+C \varepsilon^{-\frac{1}{3 \theta-1}} \exp \left(-\pi^{2} \frac{\varepsilon^{-\omega+1}}{16\left(r^{\varepsilon}(s)\right)^{2}}\right) .
$$

Proof. As for (5.23), write for $x \in \mathcal{I}_{\varepsilon}^{\text {trs }}(s) \cap \mathcal{J}_{\varepsilon}(s-\varepsilon)$

$$
\psi_{\varepsilon}(x)=\sup \left\{\mathfrak{W}_{\varepsilon}(x, s-\varepsilon)-\mathfrak{U}_{\varepsilon}, 0\right\} \geq 0 .
$$

We notice that

$$
\psi_{\varepsilon}\left(a_{k}(s-\varepsilon)+\delta_{\operatorname{loglog}}^{\varepsilon}\right)=\psi_{\varepsilon}\left(r_{\text {int }}^{\varepsilon}(s)\right)=0 .
$$

Indeed, for the first relation, we argue as in (5.26) whereas for the second, we have $\mathfrak{U}_{\varepsilon}\left(r_{\text {int }}^{\varepsilon}(s)\right)=$ ${\stackrel{\triangleright}{\mathfrak{u}_{\varepsilon, r^{\varepsilon}(s)}}}\left(r^{\varepsilon}(s)\right)=+\infty$. We extend $\psi_{\varepsilon}$ by 0 outside the interval $\mathcal{I}_{\varepsilon}^{\operatorname{trs}}(s) \cap \mathcal{J}_{\varepsilon}(s-\varepsilon)$ and derive, arguing as for (5.25),

$$
\left|\psi_{\varepsilon}(x)\right| \leq C h_{\varepsilon}^{-\frac{1}{\theta-1}} \leq C \varepsilon^{-\frac{1}{3 \theta-1}} \text { for } x \in \mathbb{R} .
$$

We introduce the cylinder $\Lambda_{\varepsilon}^{\text {trans }}(s) \equiv\left(-r_{\text {ext }}^{\varepsilon}(s), r_{\text {int }}^{\varepsilon}(s)\right) \times(s-\varepsilon, s)$ and the solution $\Psi_{\varepsilon}$ to

$$
\left\{\begin{array}{l}
\varepsilon^{\omega} \frac{\partial \Psi_{\varepsilon}}{\partial \tau}-\frac{\partial \Psi_{\varepsilon}}{\partial x^{2}}=0 \text { on } \Lambda_{\varepsilon}^{\text {trans }}(s) \\
\Phi_{\varepsilon}(x, s-\varepsilon)=\psi_{\varepsilon}(x) \text { for } x \in\left(-r_{\text {ext }}^{\varepsilon}(s), r_{\text {int }}^{\varepsilon}(s)\right) \text { and } \\
\Psi_{\varepsilon}\left(-r_{\text {ext }}^{\varepsilon}(s), \tau\right)=\Psi_{\varepsilon}\left(r_{\text {int }}^{\varepsilon}(s), \tau\right)=0 \text { for } \tau \in(s-\varepsilon, s),
\end{array}\right.
$$

so that $\Psi_{\varepsilon} \geq 0$. Arguing as for (5.29), we obtain for $\tau \in(s-\varepsilon, s)$

$$
\left|\Psi_{\varepsilon}(y, \tau)\right| \leq C \varepsilon^{-\frac{1}{3 \theta-1}} \exp \left(-\pi^{2} \varepsilon^{-\omega} \frac{(\tau-(s-\varepsilon))}{16\left(r^{\varepsilon}(s)\right)^{2}}\right) .
$$

We consider on $\Lambda_{\varepsilon}^{\text {trans }}(s)$ the function $W_{\varepsilon}^{\text {trans }}$ defined by

$$
W_{\varepsilon}^{\text {trans }}(y, \tau)=\mathfrak{U}_{\varepsilon}(y)+\Psi_{\varepsilon}(y, \tau) .
$$


It follows from Lemma 5.3 that $L_{\varepsilon}\left(W^{\text {trans }}\right)_{\varepsilon} \geq 0$, that is $W_{\varepsilon}^{\text {trans }}$ is a supersolution for $L_{\varepsilon}$ on $\Lambda_{\varepsilon}^{\text {trans }}(s)$. Consider next the subset $\Pi_{\varepsilon}^{\text {trans }}(s)$ of $\Lambda_{\varepsilon}^{\text {trans }}$ defined by

$$
\Pi_{\varepsilon}^{\text {trans }}(s) \equiv \underset{\tau \in(s-\varepsilon, s)}{\cup}\left(a_{k}(\tau)+\delta_{\log \log }^{\varepsilon}, r_{\text {int }}^{\varepsilon}(s)\right) \times\{\tau\} .
$$

We claim that

$$
W_{\varepsilon}^{\text {trans }} \geq \mathfrak{W}_{\varepsilon} \text { on } \partial_{p} \Pi_{\varepsilon}^{\text {trans }}(s) .
$$

Indeed, by construction, we have $W_{\varepsilon}^{\text {trans }}=+\infty$ on $r_{\text {int }}^{\varepsilon}(s) \times(s-\varepsilon, s)$ and $W_{\varepsilon}^{\text {trans }}(x, s-\varepsilon) \geq$ $\mathfrak{W}_{\varepsilon}(x, s-\varepsilon)$ for $x \in\left(a_{k}(s-\varepsilon)+\delta_{\log \log }^{\varepsilon}, r_{\text {int }}^{\varepsilon}(s)\right)$. Finally on $\cup_{\tau \in(s-\varepsilon, s)}\left\{a_{k}(\tau)+\delta_{\operatorname{loglog}}^{\varepsilon}\right\} \times\{\tau\}$, the conclusion (5.37) follows from estimate (47) of Lemma 2. Combining inequality (5.37) with the comparison principle, we are led to

$$
W_{\varepsilon}^{\text {trans }} \geq \mathfrak{W}_{\varepsilon} \text { on } \Pi_{\varepsilon}^{\text {trans }}(s) .
$$

Combining (5.38) with (5.36) we are led to (5.33).

Our next task is to construct a subsolution. To that aim, we rely on the symmetries of the equation, in particular the invariance $x \rightarrow-x$ and the almost oddness of the nonlinearity. To be more specific, we introduce the operator

$$
\tilde{L}_{\varepsilon}(u) \equiv \varepsilon^{\omega} \frac{\partial u}{\partial \tau}-\frac{\partial^{2} u}{\partial x^{2}}+\lambda \tilde{f}_{\varepsilon}(u)=0, \text { with } \tilde{f}_{\varepsilon}(u)=2 \theta u^{2 \theta-1}\left(1-\varepsilon^{\frac{1}{\theta-1}} u g\left(-\varepsilon^{\frac{1}{\theta-1}} u\right)\right),
$$

which has the same properties as $L_{\varepsilon}$ and consider the stationary solution $\mathfrak{u}_{\varepsilon, r^{\varepsilon}(s)}$ for $L_{\varepsilon}$ defined on $\left(-r^{\varepsilon}(s), r^{\varepsilon}(s)\right)$ by

$$
-\frac{\partial^{2} \overleftrightarrow{\mathfrak{u}}_{\varepsilon, r^{\varepsilon}(s)}}{\partial x^{2}}+\lambda f_{\varepsilon}\left(\overleftrightarrow{\mathfrak{u}}_{\varepsilon, r^{\varepsilon}(s)}\right)=0, \quad \stackrel{\triangleleft}{\mathfrak{u}}_{\varepsilon, r^{\varepsilon}(s)}\left(-r^{\varepsilon}(s)\right)=+\infty \text { and }{\stackrel{\mathfrak{u}}{\varepsilon, r^{\varepsilon}(s)}}\left(r^{\varepsilon}(s)\right)=-\infty,
$$

so that $-\mathfrak{u}_{\varepsilon, r^{\varepsilon}(s)}$ is a stationary solution to $\tilde{L}_{\varepsilon}$. Consider the function $\tilde{\mathfrak{W}}_{\varepsilon}$ defined by

$$
\tilde{\mathfrak{W}}_{\varepsilon}(x, \tau)=-\mathfrak{W}_{\varepsilon}(-x, \tau)
$$

and observe that $\tilde{L}_{\varepsilon}\left(\tilde{\mathfrak{W}}_{\varepsilon}\right)=0$. Finally, we define the interval $\left(-r_{\text {int }}^{\varepsilon}(s), r_{\text {ext }}^{\varepsilon}(s)\right)$ the function

$$
\mathfrak{V}_{\varepsilon}(x) \equiv \mathfrak{u}_{\varepsilon, r^{\varepsilon}(s)}\left(2 h_{\varepsilon}-x\right),
$$

so that $\mathfrak{V}_{\varepsilon}(x) \rightarrow-\infty$ as $x \rightarrow-r_{\text {int }}^{\varepsilon}(s)$ and $\mathfrak{V}_{\varepsilon}(x) \rightarrow+\infty$ as $x \rightarrow r_{\text {ext }}^{\varepsilon}(s)$.

Proposition 5.7. For $x \in\left(-r_{\text {int }}^{\varepsilon}(s), a_{k+1}(s)-\delta_{\text {loglog }}^{\varepsilon}\right)$ we have the inequality,

$$
\mathfrak{W}_{\varepsilon}(x, s) \geq \mathfrak{V}_{\varepsilon}(x)-C \varepsilon^{-\frac{1}{3 \theta-1}} \exp \left(-\pi^{2} \frac{\varepsilon^{-\omega+1}}{16\left(r^{\varepsilon}(s)\right)^{2}}\right) .
$$

Proof. We argue as in the proof of Proposition 5.6, replacing $L_{\varepsilon}$ by $\tilde{\varepsilon}, \mathfrak{W}_{\varepsilon}$ by $\tilde{\mathfrak{W}}_{\varepsilon}$, and $\mathfrak{U}_{\varepsilon}$ by $\tilde{\mathfrak{U}}_{\varepsilon}=-\mathfrak{u}_{\varepsilon, r^{\varepsilon}(s)}\left(\cdot-2 h_{\varepsilon}\left(s_{0}\right)\right)$. Inequality (5.40) for $\mathfrak{W}_{\varepsilon}$ is then obtained inverting relation (5.39) and from the corresponding estimate on $\tilde{\mathfrak{W}}_{\varepsilon}$.

Proof of Proposition 5.2 completed. Combining (5.33) with (5.40) we are led to

$$
\mathfrak{U}_{\varepsilon}(x)-\tilde{A}_{\varepsilon} \leq \mathfrak{W}_{\varepsilon}(x, s) \leq \mathfrak{V}_{\varepsilon}(x)+\tilde{A}_{\varepsilon},
$$

where we have set $\tilde{A}_{\varepsilon}=C \varepsilon^{-\frac{1}{3 \theta-1}} \exp \left(-\pi^{2} \frac{\varepsilon^{-\omega+1}}{16\left(r^{\varepsilon}(s)\right)^{2}}\right)$. The proof is then completed with the same arguments as in the proof of Proposition 5.1 


\subsection{Estimating the discrepancy}

\subsubsection{Linear estimates}

The purpose of this section is to provide the proof of Proposition 5.3. So far Proposition 5.1 and Proposition 5.2 provide a good approximation of $\mathfrak{W}_{\varepsilon}$ on the level of the uniform norm. However, the discrepancy involves also a first order derivative, for which we rely on the regularization property of the linear heat equation. To that aim, set

$$
\left\{\begin{array}{c}
\Lambda \equiv(-1,1) \times[0,1], \Lambda^{1 / 2} \equiv\left(-\frac{1}{2}, \frac{1}{2}\right) \times\left[\frac{3}{4}, 1\right], \text { and more generally for } \varrho>0 \\
\Lambda_{\varrho} \equiv(-\varrho, \varrho) \times\left[0, \varrho^{2}\right], \Lambda_{\varrho}^{1 / 2} \equiv\left(-\frac{1}{2} \varrho, \frac{1}{2} \varrho\right) \times\left[\frac{3}{4} \varrho^{2}, \varrho^{2}\right] .
\end{array}\right.
$$

The following standard result (see e.g. [2] Lemma A7 for a proof) is useful in our context.

Lemma 5.6. Let $u$ be a smooth real-valued function on $\Lambda$. There exists a constant $C>0$ such that

$$
\left\|u_{x}\right\|_{L^{\infty}\left(\Lambda^{1 / 2}\right)} \leq C\left(\left\|u_{t}-u_{x x}\right\|_{L^{\infty}(\Lambda)}+\|u\|_{L^{\infty}(\Lambda)}\right) .
$$

We deduce from this result the following scaled version.

Lemma 5.7. Let $\varrho>0$ and let $u$ be defined on $\Lambda_{\varrho}$. Then we have for some constant $C>0$ independent of $\varrho$

$$
\left\|u_{x}\right\|_{L^{\infty}\left(\Lambda_{\varrho}^{1 / 2}\right)}^{2} \leq C\left[\left\|u_{t}-u_{x x}\right\|_{L^{\infty}\left(\Lambda_{\varrho}\right)}\|u\|_{L^{\infty}\left(\Lambda_{\varrho}\right)}+\varrho^{-2}\|u\|_{L^{\infty}\left(\Lambda_{\varrho}\right)}^{2}\right] .
$$

Proof. The argument is parallel to the proof of Lemma A.1 in [1], which corresponds to its elliptic version. Set $h=u_{t}-u_{x x}$, let $\left(x_{0}, t_{0}\right)$ be given in $\Lambda_{\varrho}^{1 / 2}$, and let $0<\mu \leq \frac{\varrho}{2}$ be a constant to be determined in the course of the proof. We consider the function

$$
\left.v(y, \tau)=u\left(2 \mu y+x_{0}, 4 \mu^{2}(\tau-1)+t_{0}\right)\right),
$$

so that $v$ is defined on $\Lambda$ and satisfies there

$$
v_{t}-v_{y y}=\mu^{2} h\left(\left(2 \mu y+x_{0}, 4 \mu^{2}(\tau-1)+t_{0}\right)\right) \text { on } \Lambda .
$$

Applying Lemma 5.6 to $v$ we are led to

$$
\begin{aligned}
\left|v_{y}(0,1)\right| & \left.\leq C\left(\mu^{2} \| h\left(2 \mu y+x_{0}, 4 \mu^{2}(\tau-1)+t_{0}\right)\right)\left\|_{L^{\infty}(\Lambda)}+\right\| v \|_{L^{\infty}(\Lambda)}\right) \\
& \leq C\left(\mu^{2}\|h\|_{L^{\infty}\left(\Lambda_{\varrho}\right)}+\|u\|_{L^{\infty}\left(\Lambda_{\varrho}\right)}\right),
\end{aligned}
$$

so that, going back to $u$, we obtain

$$
\mu\left|u_{x}\left(x_{0}, t_{0}\right)\right| \leq C\left(\mu^{2}\left\|\left.h\right|_{L^{\infty}\left(\Lambda_{\varrho}\right)}+\right\| u \|_{L^{\infty}\left(\Lambda_{\varrho}\right)}\right) .
$$

We distinguish two cases:

Case 1: $\|u\|_{L^{\infty}} \leq \varrho^{2}\|h\|_{L^{\infty}}$. In this case we apply (5.43) with $\mu=\left(\frac{\|u\|_{L^{\infty}}}{\|h\|_{L^{\infty}}}\right)^{\frac{1}{2}}$. This yields

$$
\left|u_{y}\left(x_{0}, t_{0}\right)\right| \leq 2 C\|u\|_{L^{\infty}}^{1 / 2}\|h\|_{L^{\infty}}^{1 / 2} .
$$


Case 2: $\|u\|_{L^{\infty}} \geq \varrho^{2}\|h\|_{L^{\infty}}$. In this case we apply (5.43) with $\mu=\varrho$. We obtain

$$
\begin{aligned}
\left|u_{x}\left(x_{0}, t_{0}\right)\right| \leq & C\left(\varrho\|h\|_{L^{\infty}\left(\Lambda_{\varrho}\right)}+\varrho^{-1}\|u\|_{L^{\infty}\left(\Lambda_{\varrho}\right)}\right) \\
& \leq C\left(\|h\|_{L^{\infty}\left(\Lambda_{\varrho}\right)}^{1 / 2}\|u\|_{L^{\infty}\left(\Lambda_{\varrho}\right)}^{1 / 2}+r^{-1}\|u\|_{L^{\infty}\left(\Lambda_{\varrho}\right)}\right) .
\end{aligned}
$$

In both cases, we obtain the desired inequality.

\subsubsection{Estimating the derivative of $\mathfrak{W}_{\varepsilon}$}

Consider the general situation where we are given two functions $U$ and $U_{\varepsilon}$ defined for $(x, t) \in$ $\Lambda_{\varrho}$ and such that $L_{0}(U)=0$ and $L_{\varepsilon}\left(U_{\varepsilon}\right)=0$, where $s:=\varepsilon^{-\omega} t$, so that, in view of (5.4),

$$
\left.\left|\partial_{t}\left(U-U_{\varepsilon}\right)-\partial_{x x}\left(U-U_{\varepsilon}\right)\right| \leq C\left[\left|U-U_{\varepsilon}\right|\left(|U|^{2 \theta-2}+\left|U_{\varepsilon}\right|^{2 \theta-2}\right)+\varepsilon^{\frac{1}{\theta-1}}\left|U_{\varepsilon}\right|^{2 \theta}\right)\right] \text { on } \Lambda_{\varrho} .
$$

We deduce from (5.42) applied to the difference $U-U_{\varepsilon}$ that we have (we use the notation $\|\cdot\|=\|\cdot\|_{L^{\infty}\left(\Lambda_{\varrho}\right)}$ for simplicity)

$\left\|\left(U-U_{\varepsilon}\right)_{x}\right\|_{L^{\infty}\left(\Lambda_{\varrho}^{1 / 2}\right)}^{2} \leq C\left\|U-U_{\varepsilon}\right\|^{2}\left(\|U\|^{2 \theta-2}+\left\|U_{\varepsilon}\right\|^{2 \theta-2}+\varrho^{-2}\right)+C \varepsilon^{\frac{1}{\theta-1}}\left\|U-U_{\varepsilon}\right\|\left\|U_{\varepsilon}\right\|^{2 \theta}$.

Similarly applying (5.42) to $U$ and $U_{\varepsilon}$ we obtain

$\left\|\left(U+U_{\varepsilon}\right)_{x}\right\|_{L^{\infty}\left(\Lambda_{\varrho}^{1 / 2}\right)}^{2} \leq C\left(\|U\|^{2 \theta}+\left\|U_{\varepsilon}\right\|^{2 \theta}+\varrho^{-2}\left(\|U\|^{2}+\left\|U_{\varepsilon}\right\|^{2}\right)+\varepsilon^{\frac{1}{\theta-1}}\left(\left\|U_{\varepsilon}\right\|^{2 \theta+1}+\|U\|\left\|U_{\varepsilon}\right\|^{2 \theta}\right)\right)$,

so that

$$
\left\|\left(U^{2}-U_{\varepsilon}^{2}\right)_{x}\right\|_{L^{\infty}\left(\Lambda_{\varrho}^{1 / 2}\right)}^{2} \leq C\left[\left\|U-U_{\varepsilon}\right\|^{2} \mathcal{R}_{1}^{\varepsilon}\left(U, U_{\varepsilon}\right)+\left\|U-U_{\varepsilon}\right\| \mathcal{R}_{2}^{\varepsilon}\left(U, U_{\varepsilon}\right)\right],
$$

where we have set

$$
\left\{\begin{array}{c}
\mathcal{R}_{1}^{\varepsilon}\left(U, U_{\varepsilon}\right)=\left(\|U\|^{2 \theta-2}+\left\|U_{\varepsilon}\right\|^{2 \theta-2}+\varrho^{-2}\right)\left(\|U\|^{2 \theta}+\left\|U_{\varepsilon}\right\|^{2 \theta}+\varrho^{-2}\left(\|U\|^{2}+\left\|U_{\varepsilon}\right\|^{2}\right)\right. \\
\left.\quad+\varepsilon^{\frac{1}{\theta-1}}\left(\left\|U_{\varepsilon}\right\|^{2 \theta+1}+\|U\|\left\|U_{\varepsilon}\right\|^{2 \theta}\right)\right), \\
\mathcal{R}_{2}^{\varepsilon}\left(U, U_{\varepsilon}\right)=\varepsilon^{\frac{1}{\theta-1}}\left\|U_{\varepsilon}\right\|^{2 \theta}\left(\|U\|^{2 \theta}+\left\|U_{\varepsilon}\right\|^{2 \theta}+\varrho^{-2}\left(\|U\|^{2}+\left\|U_{\varepsilon}\right\|^{2}\right)\right. \\
\left.+\varepsilon^{\frac{1}{\theta-1}}\left(\left\|U_{\varepsilon}\right\|^{2 \theta+1}+\|U\|\left\|U_{\varepsilon}\right\|^{2 \theta}\right)\right) .
\end{array}\right.
$$

We specify next the discussion to our original situation. Thanks to the general inequality (5.45), we are in position to establish:

Proposition 5.8. If (5.21) hold and $\varepsilon$ is sufficiently small, then for any $s \in I^{\varepsilon}\left(s_{0}\right)$ and every $x \in \Theta_{k+\frac{1}{2}}^{\varepsilon}(s)$ we have the estimate

$$
\left|\left(\mathfrak{W}_{\varepsilon}\right)_{x}^{2}(x)-\lambda^{-\frac{1}{(\theta-1)}}\left(\mathfrak{u}_{r \varepsilon}^{+}(s)\right)_{x}^{2}(x)\right| \leq C \varepsilon^{\frac{1}{\theta^{2}}}
$$

Proof. We apply inequality (5.45) on the cylinder $\Lambda_{\varrho}$ with $\varrho=\frac{1}{16} d_{\mathrm{min}}^{*}\left(s_{0}\right)$ and to the functions $U(y, \tau)=\mathfrak{W}_{\varepsilon}\left(y+x, \varepsilon^{\omega} \tau+s\right)$ and $U_{\varepsilon}(y, \tau)=\mathcal{U}_{r^{\varepsilon}(s)}^{+}(y+x)$. We first estimate $\mathcal{R}_{1}$ and $\mathcal{R}_{2}$. Since we have

$$
|U(y, \tau)|+\left|U_{\varepsilon}\right| \leq C d_{\min }^{*}\left(s_{0}\right)^{-\frac{1}{\theta-1}}, \quad \text { for }(y, \tau) \in \Lambda_{\varrho},
$$


it follows that

$$
\mathcal{R}_{1}^{\varepsilon}\left(U, U_{\varepsilon}\right) \leq d_{\min }^{*}\left(s_{0}\right)^{-4-\frac{2}{\theta-1}} \text { and } \mathcal{R}_{2}^{\varepsilon}\left(U, U_{\varepsilon}\right) \leq \varepsilon^{\frac{1}{\theta-1}} d_{\min }^{*}\left(s_{0}\right)^{-4-\frac{4}{\theta-1}} .
$$

Invoking inequality (5.42) of Lemma 5.7, and combining it with (A.7) and the conclusion of Proposition 5.1, we derive the conclusion using a crude lower bound for the power of $\varepsilon$.

Similarly we obtain

Proposition 5.9. If (5.10) hold and $\varepsilon$ is sufficiently small, then for any $s \in I^{\varepsilon}\left(s_{0}\right)$ and every $x \in \Theta_{k+\frac{1}{2}}^{\varepsilon}(s)$ we have the estimate

$$
\mid\left(\mathfrak{W}_{\varepsilon}\right)_{x}^{2}(x)-\lambda^{-\frac{1}{(\theta-1)}}\left({\stackrel{\triangleright}{r^{\varepsilon}}(s)}_{x}^{2}(x) \mid \leq C \varepsilon^{\frac{1}{\theta^{2}}} .\right.
$$

Proof of Proposition 5.3 completed. The proof of Proposition 5.3 follows combining Proposition 5.8 in the attractive case and Proposition 5.9 in the repulsive case with the estimates (A.10).

\section{The motion law for prepared datas}

In this section, we present the

Proof of Proposition 6. Step 1. First, by definition of $\mathrm{L}_{0}$, assumption $\left(\mathrm{H}_{1}\right)$ and estimate (7), it follows that for fixed $\mathrm{L} \geq \mathrm{L}_{0}$, and for all $\varepsilon$ sufficiently small (depending only on $\mathrm{L}$ ),

$$
\mathfrak{D}_{\varepsilon}(s) \cap \mathrm{I}_{4 \mathrm{~L}} \subset \mathrm{I}_{\mathrm{L}} \quad \forall 0 \leq s \leq S,
$$

so that $\left(\mathcal{C}_{\mathrm{L}, S}\right)$ holds.

Step 2. Since the assumptions of Corollary 3.1 are met with the choice $s_{0}=0$ and $\mathrm{L}=\mathrm{L}_{0}$, we obtain that for $\varepsilon$ sufficiently small, $\mathcal{W} \mathcal{P}_{\varepsilon}^{\mathrm{L}_{0}}\left(\delta_{\log l o g}^{\varepsilon}, s\right)$ holds and $d_{\min }^{\varepsilon, \mathrm{L}}(s) \geq \frac{1}{2} d_{\min }^{*}(0)=$ $\frac{1}{2} \min \left\{a_{k+1}^{0}-a_{k}^{0}, k=1, \cdots, \ell_{0}-1\right\}$, for all $s \in I^{\varepsilon}(0)$, as well as the identities $J(s)=J(0)$, $\sigma_{i\left(k \pm \frac{1}{2}\right)}(s)=\sigma_{i\left(k \pm \frac{1}{2}\right)}(0)$ and $\dagger_{k}(s)=\dagger_{k}(0)$, for any $k \in J(0)$.

Step 3. We claim that for any $s_{1} \leq s_{2} \in I^{*}(0)$, we have

$$
\limsup _{\varepsilon \rightarrow 0}\left(\operatorname{dissip}_{\varepsilon}^{\mathrm{L}}\left(s_{1}, s_{2}\right)\right)=0 .
$$

Indeed, let $\mathrm{L} \geq \mathrm{L}_{0}$ be arbitrary. We know from Step 1 that $\left(\mathcal{C}_{\mathrm{L}, S}\right)$ holds provided $\varepsilon$ is sufficiently small. By Proposition 3, for $\varepsilon$ sufficiently small there exists two times $s_{1}^{\varepsilon}$ and $s_{2}^{\varepsilon}$ such that $0<s_{1}^{\varepsilon} \leq s_{1} \leq s_{2} \leq s_{2}^{\varepsilon},\left|s_{i}-s_{i}^{\varepsilon}\right| \leq \varepsilon^{\omega+1} \mathrm{~L}$ and $\mathcal{W} \mathcal{P}_{\varepsilon}^{\mathrm{L}}\left(\delta_{\log }^{\varepsilon}, s_{i}^{\varepsilon}\right)$ holds for $i=1,2$. From the second step and assumption $\left(\mathrm{H}_{1}\right)$ we infer that $\mathfrak{E}_{\varepsilon}^{\mathrm{L}}\left(s_{1}^{\varepsilon}\right)=\mathfrak{E}_{\varepsilon}^{\mathrm{L}_{0}}\left(s_{1}^{\varepsilon}\right)=\mathfrak{E}_{\varepsilon}^{\mathrm{L}_{0}}\left(s_{2}^{\varepsilon}\right)=\mathfrak{E}_{\varepsilon}^{\mathrm{L}}\left(s_{2}^{\varepsilon}\right)$. Invoking Corollary 3.1 we are therefore led to the inequality

$$
\operatorname{dissip}_{\varepsilon}^{\mathrm{L}_{0}}\left(s_{1}, s_{2}\right) \leq \operatorname{dissip}_{\varepsilon}^{\mathrm{L}}\left(s_{1}^{\varepsilon}, s_{2}^{\varepsilon}\right) \leq C M_{0}\left(\frac{\varepsilon}{\delta_{\log }^{\varepsilon}}\right)^{\omega}+C \mathrm{~L}^{-(\omega+2)}\left(s_{2}-s_{1}+2 \varepsilon^{\omega+1} \mathrm{~L}\right) .
$$

Since $\mathrm{L} \geq \mathrm{L}_{0}$ was arbitrary the conclusion (6.1) follows letting first $\varepsilon \rightarrow 0$ and then $\mathrm{L} \rightarrow \infty$. 
Step 4. In view of Corollary 3 we may find a subsequence $\left(\varepsilon_{n}\right)_{\in \mathbb{N}}$ tending to 0 such that the functions $a_{k}^{\varepsilon_{n}}(\cdot)_{n \in \mathbb{N}}$ converge uniformly as $n \rightarrow 0$ on compact subsets on $I^{\star}(0)$. Consider the cylinder

$$
\mathcal{C}_{k+\frac{1}{2}}^{*} \equiv\left[a_{k}^{0}+\frac{1}{4} d_{\min }^{*}(0), a_{k+1}^{0}-\frac{1}{4} d_{\text {min }}^{*}(0)\right] \times I^{*}(0) .
$$

It follows from Step 2 and Proposition 5.3 that

$$
\varepsilon_{n}^{-\omega} \xi_{\varepsilon_{n}}\left(\mathfrak{v}_{\varepsilon_{n}}\right) \rightarrow \lambda_{i\left(k+\frac{1}{2}\right)}^{-\frac{1}{2(\theta-1)}} r_{k+\frac{1}{2}}(s)^{-(\omega+1)} \gamma_{k} \text { as } \varepsilon_{n} \rightarrow 0, \text { for } k=1, \cdots \ell_{0}-1
$$

uniformly on every compact subset of $\mathcal{C}_{k+\frac{1}{2}}^{*}$, where $\gamma_{k}$ is defined in (5.13) and where $r_{k+\frac{1}{2}}(s)=$ $a_{k+1}(s)-a_{k}(s)$.

Step 5. As in (4.1), we consider a test function $\chi \equiv \chi_{k}$ with the following properties

$$
\left\{\begin{array}{l}
\chi \text { has compact support in }\left[a_{k}^{0}-\frac{1}{3} d_{\min }^{*}(0), a_{k}^{0}+\frac{1}{3} d_{\min }^{*}(0)\right], \\
\chi \text { is affine on the interval }\left[a_{k}^{0}-\frac{1}{4} d_{\min }^{*}(0), a_{k}^{0}+\frac{1}{4} d_{\min }^{*}(0)\right], \text { with } \chi^{\prime}=1 \text { there } \\
\|\chi\|_{L^{\infty}(\mathbb{R})} \leq C d_{\min }^{*}(0),\left\|\chi^{\prime}\right\|_{L^{\infty}(\mathbb{R})} \leq C \text { and }\left\|\chi^{\prime \prime}\right\|_{L^{\infty}(\mathbb{R})} \leq C d_{\min }^{*}(0)^{-1} .
\end{array}\right.
$$

It follows from the definition of $\chi_{k}$ that $\chi_{k}^{\prime \prime}=0$ outside $a$, and so is $\varepsilon^{-\omega} \xi_{\varepsilon}\left(\mathfrak{v}_{\varepsilon}\right) \chi_{k}^{\prime \prime}$. It follows from (6.2) that for $s_{1} \leq s_{2} \in I^{*}(0)$,

$$
\begin{aligned}
\mathfrak{F}_{\varepsilon_{n}}\left(s_{1}, s_{2}, \chi_{k}\right) \rightarrow & \left(\int_{a_{k}^{0}-\frac{1}{3} d_{\min }^{*}(0)}^{a_{k}^{0}-\frac{1}{4} d_{\min }^{*}(0)} \chi^{\prime \prime}(x) d x\right)\left(\int_{s_{1}}^{s_{2}} \lambda_{k-\frac{1}{2}}^{-\frac{1}{2(\theta-1)}} r_{k-\frac{1}{2}}(s)^{-\frac{1}{\theta-1}} \gamma_{k-\frac{1}{2}} d s\right)+ \\
& \left(\int_{a_{k}^{0}+\frac{1}{4} d_{\min }^{*}(0)}^{a_{k}^{0}+\frac{1}{3} d_{\min }^{*}} \chi^{\prime \prime}(x) d x\right)\left(\int_{s_{1}}^{s_{2}} \lambda_{k+\frac{1}{2}}^{-\frac{1}{2(\theta-1)}} r_{k+\frac{1}{2}}(s)^{-\frac{1}{\theta-1}} \gamma_{k+\frac{1}{2}} d s\right)
\end{aligned}
$$

as $\varepsilon_{n} \rightarrow 0$. Since the above two integrals containing $\chi^{\prime \prime}$ are identically equal to 1 and -1 respectively, we finally deduce from (42) combined with (6.1) and (6.3), letting $\varepsilon_{n}$ tend to 0 , that for $s_{1} \leq s_{2} \in I^{*}(0)$ we have

$\left[a_{k}\left(s_{1}\right)-a_{k}\left(s_{2}\right)\right] \mathfrak{S}_{i(k)}=\int_{s_{1}}^{s_{2}}\left(\lambda_{i\left(k-\frac{1}{2}\right)}^{-\frac{1}{2(\theta-1)}} r_{k-\frac{1}{2}}(s)^{-(\omega+1)} \gamma_{k-\frac{1}{2}}-\lambda_{i\left(k+\frac{1}{2}\right)}^{-\frac{1}{2(\theta-1)}} r_{k+\frac{1}{2}}(s)^{-(\omega+1)} \gamma_{k+\frac{1}{2}}\right) d s$,

which is nothing else than the integral formulation of the system $(\mathcal{S})$. Since the latter possesses a unique solution, the limiting points are unique and therefore convergence of the $a_{k}^{\varepsilon}$ for $s \in I^{*}(s)$ holds for the full family $\left(\mathfrak{v}_{\varepsilon}\right)_{\varepsilon>0}$.

Step 6 . We use an elementary continuation method to extend the convergence from $I^{*}(0)$ to the full interval $(0, S)$. Indeed, as long as $d_{\min }^{*}(s)$ remains bounded from below by a strictly positive constant (which holds, by definition of $S_{\max }$, as long as $s<S$ ) we may take $s$ as a new origin of times (Step 2 yields $\mathcal{W P}_{\varepsilon}^{\mathrm{L}_{0}}\left(\alpha_{1} \varepsilon, s\right)$ ) and use Steps 1 to 5 to extend the stated convergence past $s$. The proof is here completed.

\section{Clearing-out}

The purpose of this section is to provide a proof to Proposition 7. We are led to consider the situation where for some length $\mathrm{L} \geq 0$ we have

$$
\mathfrak{D}_{\varepsilon}(0) \cap[-5 \mathrm{~L}, 5 \mathrm{~L}] \subset\left[-\mathrm{K}_{0} \mathrm{~L}, \mathrm{~K}_{0} \mathrm{~L}\right]
$$


for some (small) constant $\kappa_{0} \leq \frac{1}{2}$. It follows from Theorem 2 that

$$
\mathcal{C}_{\mathrm{L}, S} \text { holds, } \quad \text { where } S=\rho_{0}\left(\frac{\mathrm{L}}{2}\right)^{\omega+2}
$$

and that for $s \in[0, S]$ we have

$$
\mathfrak{D}_{\varepsilon}(s) \cap[-4 \mathrm{~L}, 4 \mathrm{~L}] \subset\left[-\mathrm{\kappa}_{0}(s) \mathrm{L}, \mathrm{\kappa}_{0}(s) \mathrm{L}\right]
$$

where

$$
\kappa_{0}(s):=\kappa_{0}+\left(\frac{s}{\rho_{0}}\right)^{\frac{1}{\omega+2}} \frac{1}{\mathrm{~L}} .
$$

For those times $s \in[0, S]$ for which the preparedness assumption $\operatorname{WPI}_{\varepsilon}^{\mathrm{L}}\left(\alpha_{1} \varepsilon, s\right)$ holds we set

$$
\left\{\begin{array}{l}
d_{\min }^{\varepsilon,+}(s)=\min \left\{\left|a_{k+1}^{\varepsilon}(s)-a_{k}^{\varepsilon}(s)\right|, k \in J^{+}(s)\right\}, \quad \text { and } \\
d_{\min }^{\varepsilon,-}(s)=\min \left\{\left|a_{k+1}^{\varepsilon}(s)-a_{k}^{\varepsilon}(s)\right|, k \in J^{-}(s)\right\},
\end{array}\right.
$$

with $J^{ \pm}(s)=\left\{k \in\{1, \cdots, \ell(s)-1\}\right.$, s. t $\left.\epsilon_{k+\frac{1}{2}}=\mp 1\right\}$, so that $d_{\min }^{\varepsilon}(s)=\min \left\{d_{\min }^{\varepsilon,+}(s), d_{\min }^{\varepsilon,-}(s)\right\}$, with the convention that the quantities are equal to $\mathrm{L}$ in case the defining set is empty.

At first, we will focus on the case $J^{-}(s) \neq \emptyset$. The following result provides an upper bound in terms of $d_{\min }^{\varepsilon,-}(s)$ for a dissipation time for the quantized function $\mathfrak{E}_{\varepsilon}^{\mathrm{L}}$. This phenomenon is related to the cancellation of a front with its anti-front, and is the main building block for the proof of Proposition 7.

Proposition 7.1. There exist $\kappa_{1}>0, \alpha_{3}>0$, and $\mathcal{K}_{\text {col }}>0$, all depending only on $V$ and $M_{0}$, with the following properties. If (7.1) holds, if $s_{0} \in\left(\varepsilon^{\omega} \mathrm{L}^{2}, S\right)$ is such that $\mathrm{\kappa}_{0}\left(s_{0}\right) \leq \mathrm{\kappa}_{1}$, $\mathcal{W P}_{\varepsilon}^{\mathrm{L}}\left(\alpha_{3} \varepsilon, s_{0}\right)$ holds, $J^{-}\left(s_{0}\right)$ is non empty, and $s_{0}+\mathcal{K}_{\text {col }} d_{\min }^{\varepsilon,-}\left(s_{0}\right)^{\omega+2}<S$, then there exists some time $\mathcal{T}_{\text {col }}^{\varepsilon,+}\left(s_{0}\right) \in\left(s_{0}, S\right)$ such that $\mathrm{WPI}_{\varepsilon}^{\mathrm{L}}\left(\alpha_{3} \varepsilon, \mathcal{T}_{\text {col }}^{\varepsilon,+}\left(s_{0}\right)\right)$ holds,

$$
\mathfrak{E}_{\varepsilon}^{\mathrm{L}}\left(\mathcal{T}_{\text {col }}^{\varepsilon,+}\left(s_{0}\right)\right) \leq \mathfrak{E}_{\varepsilon}^{\mathrm{L}}\left(s_{0}\right)-\mu_{1},
$$

where $\mu_{1}$ is a constant introduced in Lemma 3.2, and

$$
\mathcal{T}_{\text {col }}^{\varepsilon,+}\left(s_{0}\right)-s_{0} \leq \mathcal{K}_{\text {col }}\left(d_{\text {min }}^{\varepsilon,-}\left(s_{0}\right)\right)^{\omega+2}
$$

We postpone the proof of Proposition 7.1 to after Section 7.1 below, where we will analyze more into details the attractive and repulsive forces at work at the $\varepsilon$ level. We will then prove Proposition 7.1 in Section 7.2, and finally Proposition 7 in Section 7.3.

\subsection{Attractive and repulsive forces at the $\varepsilon$ level}

In this subsection we consider the general situation where $\mathcal{C}_{\mathrm{L}, S}$ holds, for some length $\mathrm{L} \geq 0$ and some $S>0$.

In order to deal with the attractive and repulsive forces underlying annihilations or splittings, we set

$$
\mathcal{F}_{k+\frac{1}{2}}(s)=-\omega^{-1} \mathcal{B}_{k+\frac{1}{2}}\left(a_{k+1}^{\varepsilon}(s)-a_{k}^{\varepsilon}(s)\right)^{-\omega}
$$


and consider the positive functionals

$$
\mathcal{F}_{\text {rep }}^{\varepsilon}(s)=\sum_{k \in J^{+}(s)} \mathcal{F}_{k+\frac{1}{2}}(s), \mathcal{F}_{\text {att }}^{\varepsilon}(s)=-\sum_{k \in J^{-}(s)} \mathcal{F}_{k+\frac{1}{2}}(s),
$$

with the convention that the quantity is equal to $+\infty$ in case the defining set is empty. For some constants $0<\kappa_{2} \leq \kappa_{3}$ depending only on $M_{0}$, we have and $V$

$$
\left\{\begin{array}{l}
\kappa_{2} \mathcal{F}_{\text {att }}^{\varepsilon}(s)^{-\frac{1}{\omega}} \leq d_{\text {min }}^{\varepsilon,-}(s) \leq \kappa_{3} \mathcal{F}_{\text {att }}^{\varepsilon}(s)^{-\frac{1}{\omega}} \\
\kappa_{2} \mathcal{F}_{\text {rep }}^{\varepsilon}(s)^{-\frac{1}{\omega}} \leq d_{\text {min }}^{\varepsilon,+}(s) \leq \kappa_{3} \mathcal{F}_{\text {rep }}^{\varepsilon}(s)^{-\frac{1}{\omega}}
\end{array}\right.
$$

Let $s_{0} \in\left[\varepsilon^{\omega} \mathrm{L}^{2}, S\right]$ be such that

$$
\mathcal{W P}_{\varepsilon}^{\mathrm{L}}\left(\alpha_{2} \varepsilon, s_{0}\right) \text { holds } \quad \text { and } \quad d_{\text {min }}^{\varepsilon, \mathrm{L}}\left(s_{0}\right) \geq 16 \mathfrak{q}_{1}\left(\alpha_{2}\right) \varepsilon .
$$

We consider as in Corollary 3.3 the stopping time

$$
\mathcal{T}_{0}^{\varepsilon}\left(\alpha_{2}, s_{0}\right)=\max \left\{s_{0}+\varepsilon^{2+\omega} \leq s \leq S \quad \text { s.t. } \quad d_{\min }^{\varepsilon, \mathrm{L}}\left(s^{\prime}\right) \geq 8 \mathfrak{q}_{1}\left(\alpha_{2}\right) \varepsilon \quad \forall s^{\prime} \in\left[s_{0}+\varepsilon^{\omega+2}, s\right]\right\},
$$

and for simplicity we will write $\mathcal{T}_{0}^{\varepsilon}\left(s_{0}\right) \equiv \mathcal{T}_{0}^{\varepsilon}\left(\alpha_{2}, s_{0}\right)$. In view of (7.8) and the statement of Corollary 3.3,

$$
\mathcal{W P}_{\varepsilon}^{\mathrm{L}}\left(\alpha_{1} \varepsilon, s\right) \text { holds } \quad \forall s \in \mathcal{I}_{0}^{\varepsilon}\left(s_{0}\right) \equiv\left[s_{0}+\varepsilon^{2+\omega}, \mathcal{T}_{0}^{\varepsilon}\left(s_{0}\right)\right] .
$$

The functionals $\mathcal{F}_{\text {att }}^{\varepsilon}$ and $\mathcal{F}_{\text {rep }}^{\varepsilon}$ are in particular well defined and continuous on the interval of time $\mathcal{I}_{0}^{\varepsilon}\left(s_{0}\right)$ with $J^{+}(s)=J^{+}\left(s_{0}\right)$ and $J^{-}(s)=J^{-}\left(s_{0}\right)$ for all $s$ that interval. Note that the attractive forces are dominant when $d_{\min }^{\varepsilon,-}(s) \leq d_{\min }^{\varepsilon,+}(s)$ and in contrario the repulsive forces are dominant when $d_{\min }^{\varepsilon,+}(s) \leq d_{\min }^{\varepsilon,-}(s)$.

We first focus on the attractive case, and for $s \in \mathcal{I}_{0}^{\varepsilon}\left(s_{0}\right)$, we introduce the new stopping times

$$
\mathcal{T}_{1}^{\varepsilon}(s)=\inf \left\{s \leq s^{\prime} \leq \mathcal{T}_{0}^{\varepsilon}\left(s_{0}\right), F_{\text {att }}\left(s^{\prime}\right) \geq v_{1}^{\omega} F_{\text {att }}(s) \text { or } s^{\prime}=\mathcal{T}_{0}^{\varepsilon}\left(s_{0}\right)\right\},
$$

where $v_{1}=10 \kappa_{3}^{2} \kappa_{2}^{-2}$, so that $v_{1}>10$ and $\mathcal{T}_{1}^{\varepsilon}(s) \leq \mathcal{T}_{0}^{\varepsilon}\left(s_{0}\right)$. In view of $(7.7)$, we have

$$
\frac{1}{10}\left(\frac{\kappa_{2}}{\kappa_{3}}\right)^{3} d_{\min }^{\varepsilon,-}(s) \leq d_{\min }^{\varepsilon,-}\left(\mathcal{T}_{1}^{\varepsilon}(s)\right)
$$

and if $\mathcal{T}_{1}^{\varepsilon}(s)<\mathcal{T}_{0}^{\varepsilon}\left(s_{0}\right)$ then

$$
d_{\text {min }}^{\varepsilon,-}\left(\mathcal{T}_{1}^{\varepsilon}(s)\right) \leq \frac{1}{10} \frac{\kappa_{2}}{\kappa_{3}} d_{\min }^{\varepsilon,-}(s) \leq \frac{1}{10} d_{\min }^{\varepsilon,-}(s) .
$$

The next result provides an upper bound on $\mathcal{T}_{1}^{\varepsilon}(s)-s$. Central in our argument is Proposition 6 , which we use combined with various arguments by contradiction. We have

Proposition 7.2. There exists $\beta_{0}>0$, depending only on $V$ and $M_{0}$, with the following properties. If $J^{-}\left(s_{0}\right) \neq \emptyset, \hat{s} \in \mathcal{I}_{0}^{\varepsilon}\left(s_{0}\right)$ and

$$
\beta_{0} \varepsilon \leq d_{\min }^{\varepsilon,-}(\hat{s}) \leq d_{\min }^{\varepsilon,+}(\hat{s}),
$$


then we have

$$
\mathcal{T}_{1}^{\varepsilon}(\hat{s})-\hat{s} \leq \mathcal{K}_{0}\left(d_{\min }^{\varepsilon,-}(\hat{s})\right)^{\omega+2},
$$

where $\mathcal{K}_{0}$ is defined in (19), and moreover if $\mathcal{T}_{1}^{\varepsilon}(\hat{s})<S$ then

$$
d_{\min }^{\varepsilon,-}\left(\mathcal{T}_{1}^{\varepsilon}(\hat{s})\right) \leq d_{\min }^{\varepsilon,+}\left(\mathcal{T}_{1}^{\varepsilon}(\hat{s})\right) .
$$

Proof. Up to a translation of times we may first assume that $\hat{s}=0$, which eases somewhat the notations. We then argue by contraction and assume that the conclusion is false, that is, there does not exist any such constant $\beta_{0}$, no matter how large it is chosen, such that the conclusion holds. Taking $\beta_{0}=n$, this means that given any $n \in \mathbb{N}_{*}$ there exist some $0<\varepsilon_{n} \leq 1$, a solution $v_{n}$ to $(\mathrm{PGL})_{\varepsilon_{n}}$ such that $\mathcal{E}_{\varepsilon_{n}}\left(v_{n}\right) \leq M_{0}$, such that $\operatorname{WP}_{\varepsilon_{n}}^{\mathrm{L}_{0}}\left(\alpha_{1} \varepsilon_{n}, 0\right)$ holds, such that

$$
n \varepsilon_{n} \leq d_{\min }^{\varepsilon_{n},-}(0)=d_{\min }^{\varepsilon_{n}}(0) \leq d_{\min }^{\varepsilon_{n},+}(0),
$$

and such that one of the conclusion fails, that is such that either

$$
\mathcal{T}_{1}^{n} \equiv \mathcal{T}_{1}^{\varepsilon_{n}}(0)>\mathcal{K}_{0}\left(d_{\text {min }}^{\varepsilon,-}(0)\right)^{\omega+2},
$$

or

$$
d_{\min }^{\varepsilon,-}\left(\mathcal{T}_{1}^{n}\right)>d_{\min }^{\varepsilon,+}\left(\mathcal{T}_{1}^{n}\right)
$$

Setting $S_{0}^{n}=\mathcal{K}_{0}\left(d_{\min }^{\varepsilon_{n},-}(0)\right)^{\omega+2}$, relation (7.15) may be rephrased as

$$
F_{\text {att }}^{n}(s) \leq v_{1}^{\omega} F_{\text {att }}^{n}(0) \text { and } d_{\min }^{\varepsilon_{n}}(s) \geq 8 \mathfrak{q}_{1}\left(\alpha_{2}\right) \varepsilon_{n} \text { for any } s \in\left[0, S_{0}^{n}\right],
$$

where the superscripts $n$ refer to the corresponding functionals computed for the map $v_{n}$. Passing possibly to a subsequence, we may therefore assume that one at least of the properties (7.17) or (7.16) holds for any $n \in \mathbb{N}_{*}$. Also, passing possibly to a further subsequence, we may assume that the total number of fronts of $v_{n}(0)$ inside $[-\mathrm{L}, \mathrm{L}]$ is constant, equal to a number $\ell$, denote $a_{1}^{n}(s), \cdots, a_{\ell}^{n}(s)$ the corresponding front points, for $s \in\left[0, \mathcal{T}_{1}^{n}\right]$, and set $d_{n}^{-}(s)=d_{\min }^{\varepsilon_{n},-}(s), d_{n}^{+}(s)=d_{\min }^{\varepsilon_{n},+}(s), d_{n}(s)=d_{\min }^{\varepsilon_{n}}(s)$.

In order to obtain a contradiction we shall make use of the scale invariance of the equation: if $v_{\varepsilon}$ is a solution to $(\mathrm{PGL})_{\varepsilon}$ then the map $\tilde{v}_{\tilde{\varepsilon}}(x, t)=v_{\varepsilon}\left(r x, r^{2} t\right)$ is a solution to (PGL) $\tilde{\varepsilon}$ with $\tilde{\varepsilon}=r^{-1} \varepsilon$. As scaling factor $r_{n}$, we choose $r_{n}=d_{\min }^{\varepsilon_{n},-}(0) \geq n \varepsilon_{n}$ and set

$$
\tilde{v}_{n}(x, t)=v_{n}\left(r_{n} x, r_{n}^{2} t\right), \quad \tilde{\mathfrak{v}}_{n}(x, \tau)=\tilde{v}_{n}\left(x, \tilde{\varepsilon}_{n}^{-\omega} \tau\right),
$$

so that $\tilde{v}_{n}$ is a solution to $(\mathrm{PGL}) \tilde{\varepsilon}_{n}$ satisfying $\mathcal{W} P_{\tilde{\varepsilon}_{n}}^{L_{n}}\left(\alpha_{2} \tilde{\varepsilon}_{n}, 0\right)$ with $L_{n}=r_{n}^{-1} \mathrm{~L}$ and

$$
\tilde{\varepsilon}_{n}=\left(r_{n}\right)^{-1} \varepsilon_{n}=\left(d_{\min }^{\varepsilon_{n},-}(0)\right)^{-1} \varepsilon_{n} \leq \frac{1}{n}, \text { hence we have } \varepsilon_{n} \rightarrow 0 \text { as } n \rightarrow+\infty .
$$

The points $\tilde{a}_{1}^{n}(s)=r_{n}^{-1} a_{1}^{n}\left(r_{n}^{-(2+\omega)} s\right), \cdots, \tilde{a}_{\ell}^{n}(s)=r_{n}^{-1} a_{\ell}^{n}\left(r_{n}^{-(2+\omega)} s\right)$ are the front points of $\tilde{\mathfrak{v}}_{n}$. Let $\tilde{d}_{n}^{-}, \tilde{d}_{n}^{+}, \tilde{d}_{n}$ be the quantities corresponding to $d_{\text {min }}^{\varepsilon,-}, d_{\min }^{\varepsilon,+}, d_{\min }^{\varepsilon}$ for $\tilde{\mathfrak{v}}_{n}$, so that

$$
\tilde{d}_{n}^{-}(s)=r_{n}^{-1} d_{n}^{-}\left(r_{n}^{-(2+\omega)} s\right), \quad \tilde{d}_{n}^{+}(s)=r_{n}^{-1} d_{n}^{+}\left(r_{n}^{-(2+\omega)} s\right), \quad \text { and } \tilde{d}_{n}(s)=r_{n}^{-1} d_{n}\left(r_{n}^{-(2+\omega)} s\right),
$$

and notice that $d_{n}^{-}(0)=d_{n}(0)=1$. We next distinguish the following two complementing cases. 
Case 1: (7.17) holds for all $n \in \mathbb{N}_{*}$. It follows from assumption (7.17) that $\mathrm{WP}_{\tilde{\varepsilon}_{n}}^{L_{n}}\left(\alpha_{1} \tilde{\varepsilon}_{n}, \tau\right)$ holds for every $\tau \in\left(0, \tilde{S}_{1}^{n}\right)$, where $\tilde{\mathcal{S}}_{1}^{n}=r_{n}^{-(2+\omega)} S_{0}^{n}=\mathcal{K}_{0}$. Let $k_{0} \in\{1, \cdots, \ell\}$ be such that

$$
a_{k_{0}+1}^{n}(0)-a_{k_{0}}^{n}(0)=d_{\min }^{\varepsilon_{n},-}(0) .
$$

Upon a translation if necessary, we may also assume that $a_{k_{0}}^{n}(0)=0$ so that $a_{k_{0}+1}^{n}(0)=d_{\min }^{\varepsilon_{n},-}(0)$. We denote by $\tilde{\mathcal{F}}_{\text {att }}^{n}$ the functional $\mathcal{F}_{\text {att }}^{\varepsilon}$ computed for the front points of $\tilde{\mathfrak{v}}_{n}$, so that

$$
\tilde{\mathcal{F}}_{\mathrm{att}}^{n}\left(r_{n}^{-(2+\omega)} s\right)=r_{n}^{(2+\omega)} \mathcal{F}_{\mathrm{att}}^{n}(s) .
$$

By construction we have

$$
\tilde{a}_{k_{0}}^{n}(0)=0 \text { and } \tilde{a}_{k_{0}+1}^{n}(0)=1=\tilde{d}_{n}^{-}(0) .
$$

Since $\tilde{\varepsilon}_{n} \rightarrow 0$ as $n \rightarrow \infty$, we may implement part of the already established asymptotic analysis for $(\mathrm{PGL})_{\varepsilon}$ on the sequence $\left(\tilde{v}_{n}\right)_{n \in \mathbb{N}}$. First, passing possibly to a subsequence, we may assume that for some subset $\tilde{J} \subset J(0)$ the points $\left\{\tilde{a}_{k}(0)\right\}_{k \in \tilde{J}}$ converge to some finite limits $\left\{\tilde{a}_{k}^{0}\right\}_{k \in \tilde{J}}$, whereas the points with indices in $J(0) \backslash \tilde{J}$ diverge either to $+\infty$ or to $-\infty$. We choose $\tilde{\mathrm{L}} \geq 1$ so that

$$
\underset{k \in \tilde{J}}{\cup}\left\{\tilde{a}_{k}^{0}\right\} \subset\left[-\frac{\tilde{\mathrm{L}}}{2}, \frac{\tilde{\mathrm{L}}}{2}\right]
$$

In view of (7.19), we have $\tilde{a}_{k_{0}}(0)=0, \tilde{a}_{k_{0}+1}=1$ and $\inf \left\{\left|\tilde{a}_{k+1}(0)-\tilde{a}_{k}(0)\right|, k \in \tilde{J}\right\}=1$. We are hence in position to apply the convergence result stated in Proposition 6 to the sequence $\left(\tilde{\mathfrak{v}}_{n}(\cdot)\right)_{n \in \mathbb{N}}$. It states that the front points $\left(\tilde{a}_{k}^{n}(\tau)\right)_{k \in J_{0}}$ which do not escape at infinity converge to the solution $\left(\tilde{a}_{k}(\cdot)\right)_{k \in \tilde{J}}$ of the ordinary differential equation $(\mathcal{S})$ supplemented with the corresponding initial values $\left(\tilde{a}_{k}(0)\right)_{k \in \tilde{J}}$, uniformly in time on every compact subset of $\left(0, \tilde{S}_{\max }\right)$, where $\tilde{S}_{\max }$ denotes the maximal time of existence for the solution. In particular, we have

$$
\left\{\begin{array}{l}
\tilde{d}_{n}^{-}(\tau) \rightarrow \delta_{\tilde{a}}^{-}(\tau), \text { uniformly on every compact subset of }\left(0, \tilde{S}_{\max }\right), \\
\limsup _{n \rightarrow+\infty} \tilde{F}_{\text {att }}^{n}(\tau) \geq F_{\text {att }}(\tilde{a}(\tau)) \text { forevery } \tau \in\left(0, \tilde{S}_{\max }\right),
\end{array}\right.
$$

the presence of the limsup being related to the fact that some points might escape at infinity so that the limiting values of the functionals are possibly smaller. We use next the properties of the differential equation $(\mathcal{S})$ established in Appendix B. We first invoke Proposition 1 which asserts that $\tilde{S}_{\max } \leq \mathcal{K}_{0}$ and that

$$
F_{\text {att }}(\tilde{a}(\tau)) \rightarrow+\infty \text { as } \tau \rightarrow \tilde{S}_{\max }
$$

Hence, there exists some $\tau_{1} \in\left(0, \tilde{S}_{\text {max }}\right) \subset\left(0, \mathcal{K}_{0}\right)$ such that, if $n$ is sufficiently large, then

$$
\tilde{\mathcal{F}}_{\text {att }}^{n}\left(\tau_{1}\right)>v_{1}^{\omega} \tilde{\mathcal{F}}_{\text {att }}^{n}(0) .
$$

Going back to the original time scale, this yields $\mathcal{F}_{\text {att }}^{n}\left(r_{n}^{\omega+2} \tau_{1}\right)>v_{1}^{\omega} \mathcal{F}_{\text {att }}^{n}(0)$. Since $r_{n}^{\omega+2} \tau_{1} \in$ $\left(0, r_{n}^{2+\omega} \mathcal{K}_{0}\right)=\left(0, S_{0}^{n}\right)$ this contradicts $(7.17)$ and completes the proof in Case 1.

Case 2: (7.16) holds for all $n \in \mathbb{N}_{*}$. We consider an arbitrary index $j \in J^{+}$. As above, translating the origin, we may assume without loss of generally that $a_{j}^{n}(0)=0$. We also 
define the map $\mathfrak{v}_{n}$ as in Case 1, according to the same scaling as described in (7.18), the only difference being that the origin has been shifted differently. With similar notations, we have

$$
\tilde{a}_{j}^{n}(0)=0 \text { and } \tilde{a}_{j+1}^{n}(0) \geq 1=\tilde{d}_{n}^{-}(0) .
$$

Passing possibly to a further subsequence, we may assume that the front points at time 0 converge to some limits in $\overline{\mathbb{R}}$ denoted $\tilde{a}_{k}(0)$. We are hence again in position to apply the convergence result of Proposition 6, so that the front points $\left(\tilde{a}_{k}^{n}(s)\right)_{k \in J_{j}}$ which do not escape at infinity converge to the solution $\left(\tilde{a}_{k}(\cdot)\right)_{k \in J_{j}}$ of the ordinary differential equation $(\mathcal{S})$ supplemented with the corresponding initial values $\left(\tilde{a}_{k}(0)\right)_{k \in J_{j}}$, uniformly in time on every compact subset of $\left(0, \tilde{S}_{\max }^{\prime}\right)$, where $\tilde{S}_{\max }^{\prime}$ denotes the (new) maximal time of existence for the solution. It follows from assumption (7.39), Theorem 1 and scaling that $0<\tilde{\mathcal{T}}_{1} \equiv \lim \inf \tilde{\mathcal{T}}_{1}^{n} \leq \tilde{S}_{\max }^{\prime}$. We claim that, for any $\tau \in\left(0, \tilde{\mathcal{T}}_{1}\right)$, and for sufficiently large $n$, we have

$$
\left|\tilde{a}_{j}^{n}(\tau)-\tilde{a}_{j+1}^{n}(\tau)\right| \geq \frac{\kappa_{2}}{2 \kappa_{3}} .
$$

This is actually a property of the differential equation $(\mathcal{S})$. We have indeed, in view of Proposition B.1, $0<F_{\text {rep }}(\tilde{a}(\tau)) \leq F_{\text {rep }}(\tilde{a}(0))$, so that it follows from $(7.7)$ that

$$
\left|\tilde{a}_{j}(\tau)-\tilde{a}_{j+1}(\tau)\right| \geq \frac{\kappa_{2}}{\kappa_{3}},
$$

which yields (7.21) taking the convergence into account. Since (7.21) holds for any $j$, we deduce that

$$
d_{\min }^{\varepsilon_{n},+}\left(\mathcal{T}_{1}^{n}\right) \geq \frac{\kappa_{2}}{2 \kappa_{3}} d_{\min }^{\varepsilon_{n},-}(0)
$$

and therefore by (7.16) we have

$$
d_{\text {min }}^{\varepsilon_{n}}\left(\mathcal{T}_{1}^{n}\right)=d_{\min }^{\varepsilon_{n},-}\left(\mathcal{T}_{1}^{n}\right) \geq d_{\min }^{\varepsilon_{n},+}\left(\mathcal{T}_{1}^{n}\right) \geq \frac{\kappa_{2}}{2 \kappa_{3}} d_{\min }^{\varepsilon_{n},-}(0) \geq \frac{\kappa_{2}}{2 \kappa_{3}} n \varepsilon .
$$

For $n$ sufficiently large, this implies that $\mathcal{T}_{1}^{n}<\mathcal{T}_{0}^{n}$, and therefore from (7.10) we have

$$
d_{\min }^{\varepsilon_{n},-}\left(\mathcal{T}_{1}^{n}\right) \leq \frac{1}{10} \frac{\kappa_{2}}{\kappa_{3}} d_{\min }^{\varepsilon_{n},-}(0),
$$

which is in contradiction with (7.22).

We turn now to the case where $d_{\min }^{\varepsilon,+}(s) \leq d_{\min }^{\varepsilon,-}(s)$. In order to handle the repulsive forces at work, for $s \in \mathcal{I}_{0}^{\varepsilon}\left(s_{0}\right)$ we introduce the new stopping times

$$
\mathcal{T}_{2}^{\varepsilon}(s)=\inf \left\{s \leq s^{\prime} \leq \mathcal{T}_{0}^{\varepsilon}\left(s_{0}\right), \mathcal{F}_{\text {rep }}^{\varepsilon}\left(s^{\prime}\right) \leq v_{2}^{\omega} \mathcal{F}_{\text {rep }}^{\varepsilon}(s) \text { or } s^{\prime}=\mathcal{T}_{0}^{\varepsilon}\left(s_{0}\right)\right\}
$$

where $v_{2}=\frac{\kappa_{2}^{2}}{10 \kappa_{3}^{2}}$, so that $v_{2}<1$. Notice that, in view of $(7.7)$, we have, if $\mathcal{T}_{2}^{\varepsilon}(s)<\mathcal{T}_{0}^{\varepsilon}\left(s_{0}\right)$,

$$
d_{\min }^{\varepsilon,+}\left(\mathcal{T}_{2}^{\varepsilon}(s)\right) \geq v_{2}^{-1} \frac{\kappa_{2}}{\kappa_{3}} d_{\min }^{\varepsilon,+}(s) \geq 10 d_{\min }^{\varepsilon,+}(s) .
$$

With $\mathcal{S}_{1}$ introduced in Proposition 1 , we set

$$
\mathcal{K}_{1}=\mathcal{S}_{1}^{-\omega}\left(\frac{2 \kappa_{3}}{\kappa_{2} \mathcal{v}_{2}}\right)^{\omega+2} \text {. }
$$


Proposition 7.3. There exists $\beta_{1}>0$, depending only on $V$ and $M_{0}$, with the following properties. If $J^{+}\left(s_{0}\right) \neq \emptyset, \hat{s} \in \mathcal{I}_{0}^{\varepsilon}\left(s_{0}\right)$ and

$$
\beta_{1} \varepsilon \leq d_{\min }^{\varepsilon,+}(\hat{s}) \leq d_{\min }^{\varepsilon,-}(\hat{s}),
$$

then we have

$$
\mathcal{T}_{2}^{\varepsilon}(\hat{s})-\hat{s} \leq \mathcal{K}_{1}\left(d_{\min }^{\varepsilon,+}(\hat{s})\right)^{\omega+2}
$$

and if $\mathcal{T}_{2}^{\varepsilon}(\hat{s})<S$ then $\mathcal{T}_{2}^{\varepsilon}(\hat{s})<\mathcal{T}_{0}^{\varepsilon}\left(s_{0}\right)$ and for any $s \in\left[\hat{s}, \mathcal{T}_{2}^{\varepsilon}(\hat{s})\right]$, we have

$$
d_{\min }^{\varepsilon}(s) \geq \frac{1}{2} \mathcal{S}_{2} d_{\min }^{\varepsilon,+}(\hat{s}),
$$

and

$$
\mathcal{F}_{\text {att }}^{\varepsilon}(s)^{-\frac{1}{\omega}} \leq \mathcal{F}_{\text {att }}^{\varepsilon}(\hat{s})^{-\frac{1}{\omega}}+\frac{1}{\kappa_{3}}\left(d_{\min }^{\varepsilon,+}(\hat{s})\right),
$$

where $\mathcal{S}_{2}$ is defined in Proposition 1 and $\kappa_{3}$ is defined in (7.7).

Proof. The argument possesses strong similarities with the proof of Proposition 7.2, we therefore just sketch its main points, in particular relying implicitly on the notations introduced there, as far as this is possible. By translation in time we also assume that $\hat{s}=0$ and argue by contradiction assuming that for any $n \in \mathbb{N}_{*}$ there exist some $0<\varepsilon_{n} \leq 1$, a solution $v_{n}$ to $(\mathrm{PGL})_{\varepsilon_{n}}$ such that $\mathcal{E}_{\varepsilon_{n}}\left(v_{n}\right) \leq M_{0}, \mathcal{W} P_{\varepsilon_{n}}^{\mathrm{L}}\left(\alpha_{1} \varepsilon_{n}, 0\right)$ holds, such that $n \varepsilon_{n} \leq d_{n}^{+}(0)$, and such that either, we have for any $s \in\left(0, S_{1}^{n}\right)$, where $S_{1}^{n}=\mathcal{K}_{1} d_{n}^{+}(0)^{\omega+2}, d_{n}(s) \geq 8 \mathfrak{q}\left(\alpha_{2}\right) \varepsilon_{n}$ and

$$
\kappa_{3}^{\omega}\left(d_{n}^{+}\left(s^{\prime}\right)\right)^{-\omega} \geq F_{\text {rep }}^{n}\left(s^{\prime}\right) \geq v_{2}^{\omega} F_{\text {rep }}^{n}(0) \geq v_{2}^{\omega} \kappa_{2}^{\omega}\left(d_{n}^{+}(0)\right)^{-\omega}
$$

or, there is some $\tau_{n} \in\left(0, \mathcal{T}_{2}^{n}\right)$ such that

$$
d_{\min }^{\varepsilon,+}\left(\tau_{n}\right)<\frac{1}{2} \mathcal{S}_{2} d_{\min }^{\varepsilon,+}(s)
$$

or

$$
\mathcal{F}_{\text {att }}^{n}\left(\tau_{n}\right)^{-\frac{1}{\omega}}<\mathcal{F}_{\text {att }}^{n}(0)^{-\frac{1}{\omega}}+\frac{1}{32 \kappa_{3}}\left(d_{n}^{+}(s)\right) .
$$

As in (7.18), but with a different scaling $r_{n}$ we set

$$
r_{n}=d_{\min }^{\varepsilon_{n},+}(0) \geq n \varepsilon_{n}, \tilde{v}_{n}(x, t)=v_{n}\left(r_{n} x, r_{n}^{2} t\right), \text { and } \tilde{\mathfrak{v}}_{n}(x, s)=\tilde{v}_{n}\left(x, \tilde{\varepsilon}_{n}^{-\omega} s\right) .
$$

We verify that $\tilde{v}_{n}$ is a solution to (PGL) $\tilde{\varepsilon}_{n}$ with $\tilde{\varepsilon}_{n}=\left(r_{n}\right)^{-1} \varepsilon_{n} \rightarrow 0$ as $n \rightarrow \infty$ and that the points $\tilde{a}_{k}^{n}(\tau)=r_{n}^{-1} a_{k}^{n}\left(r_{n}^{-(2+\omega)} \tau\right)$ for $k \in J$, are the front points of $\tilde{\mathfrak{v}}_{n}$. We distinguish three cases, which are complementing going if necessary to subsequences.

Case 1: (7.29) holds, for any $n \in \mathbb{N}$. It follows $\mathcal{W P}_{\tilde{\varepsilon}_{n}}^{L_{n}}\left(\alpha_{1} \tilde{\varepsilon}_{n}, \tau\right)$ holds for every $\tau \in\left(0, \tilde{\mathcal{S}}_{1}^{n}\right)$, where $\tilde{\mathcal{S}}_{1}^{n}=r_{n}^{-(2+\omega)} \mathcal{S}_{1}^{n}=\mathcal{K}_{1}$. Let $j$ be an arbitrary index in $J^{+}$. Translating if necessary the origin, we may assume that $a_{j}^{n}(0)=0$ so that $a_{j+1}^{n}(0) \geq d_{n}^{+}(0) \geq n \varepsilon_{n}$ and hence $\tilde{a}_{j+1}^{n}(0)-\tilde{a}_{j}^{n}(0) \geq 1$. Since $\tilde{\varepsilon}_{n} \rightarrow 0$ as $n \rightarrow \infty$, we may implement part of the already established asymptotic analysis for $(\mathrm{PGL})_{\varepsilon}$ on the sequence $\left(\tilde{v}_{n}\right)_{n \in \mathbb{N}}$. First, passing possibly to a subsequence, we may assume that for some subset $\tilde{J} \subset J(0)$ the points $\left\{\tilde{a}_{k}(0)\right\}_{k \in \tilde{J}}$ converge to some finite limits $\left\{\tilde{a}_{k}^{0}\right\}_{k \in \tilde{J}}$, whereas the points with indices in $J(0) \backslash \tilde{J}$ diverge either to 
$+\infty$ or to $-\infty$. We choose $\tilde{\mathrm{L}} \geq 1$ so that (7.20) holds. It follows from Proposition 6 that for $\tau \in\left(0, \mathcal{K}_{1}\right)$, we have

$$
\left|\tilde{a}_{j+1}^{n}(\tau)-\tilde{a}_{j}^{n}(\tau)\right| \rightarrow\left|\tilde{a}_{j+1}(\tau)-\tilde{a}_{j}(\tau)\right| \geq\left(\mathcal{S}_{1} \tau+\mathcal{S}_{2} \delta_{\tilde{a}}^{+}(0)^{\omega+2}\right)^{\frac{1}{\omega+2}}=\left(\mathcal{S}_{1} \tau+\mathcal{S}_{2}\right)^{\frac{1}{\omega+2}}
$$

as $n \rightarrow \infty$, where the last inequality is a consequence of Proposition 1. Taking the infimum over $J^{+}$, we obtain, for $n$ sufficiently large

$$
\tilde{d}_{n}^{+}(\tau)=\inf _{j \in J^{+}}\left|\tilde{a}_{j+1}^{n}(\tau)-\tilde{a}_{j}^{n}(\tau)\right| \geq \frac{1}{2}\left(\mathcal{S}_{1} \tau+\mathcal{S}_{2}\right)^{\frac{1}{\omega+2}} \geq \frac{1}{2}\left(\mathcal{S}_{1} \tau\right)^{\frac{1}{\omega+2}}, \forall \tau \in\left(0, \mathcal{K}_{1}\right),
$$

On the other hand, going back to (7.29), with the same notation as in Proposition 7.2, we are led to the inequality

$$
\tilde{d}_{n}^{+}(\tau) \leq \kappa_{3} \kappa_{2}^{-1} v_{2}^{-1} \text { for } \tau \in\left(0, \mathcal{K}_{1}\right) .
$$

In view of our choice (7.24) of $\mathcal{K}_{1}$, relations (7.33) and (7.34) are contradictory for $\tau$ close to $\mathcal{K}_{1}$ yielding hence a contradiction in Case 1.

Case 2: (7.29) does not hold, but (7.30) holds, for any $n \in \mathbb{N}$. The argument is almost identical, we conclude again thanks to (7.33) but keeping $\mathcal{S}_{2}$ instead of $\mathcal{S}_{1} \tau$ in its last inequality. Case 3: (7.29) does not hold but (7.31) holds, for any $n \in \mathbb{N}$. As in the proof of Proposition 7.2 , we conclude that $0<\tilde{\mathcal{T}}_{2} \equiv \liminf _{n \rightarrow+\infty} \tilde{\mathcal{T}}_{2}{ }^{n}$. This situation is slightly more delicate than the ones analyzed so far, and we have to track also the fronts escaping possibly at infinity. Up to a subsequence, we may assume that the set $J$ is decomposed as a disjoint union of clusters $J=\bigcup_{i=1}^{q} J_{p}$ where each of the sets $J_{p}$ is an ordered set of $m_{p}+1$ consecutive points, that is $J_{p}=\left\{k_{p}, k_{p}+1, \cdots k_{p}+m_{p}\right\}$ and such that the two following properties holds:

- There exists a constant $C>0$ independent of $n$ such that

$$
\left|\tilde{a}_{k_{p}}^{n}(0)-\tilde{a}_{k_{p}+r}(0)\right| \leq C \text { for any } p \in\{1, \cdots, q\} \text { and any } r \in\left\{k_{p}, \cdots, m_{p}\right\}
$$

- For $1 \leq p_{1}<p_{2} \leq q$, we have $\tilde{a}_{k_{p_{2}}}^{n}-\tilde{a}_{k_{p_{1}}}^{n} \rightarrow+\infty$.

For a given $p \in\{1, \cdots, q\}$, translating if necessary the origin, we may assume that $\tilde{a}_{k_{p}}^{n}(0)=0$, and passing possibly to a further subsequence, that the front points at time 0 converge as $n \rightarrow+\infty$ to some limits denoted $\tilde{a}_{p, k}(0)$, for $k \in\left\{k_{p}, \cdots, k_{p}+m_{p}\right\}$. Notice that, as an effect of the scaling, all other front points diverge to infinity, in the chosen frame. We apply now Proposition 6 to this cluster of points : it yields uniform convergence, for $k \in\left\{k_{p}, \cdots, k_{p}+m_{p}\right\}$ of the front points $\tilde{a}_{k}^{n}(\cdot)$ to the solution $\tilde{a}_{p, k}(\cdot)$ of the differential equation $(\mathcal{S})$ supplemented with the initial time conditions $\tilde{a}_{p, k}(0)$ defined above. If $F_{\text {att }}^{p}$ denotes the functional $F_{\text {att }}$ defined in (7.6) restricted to the points of the cluster $J_{p}$, we have in view of (B.17)

$$
\frac{d}{d \tau} F_{\text {att }}^{p}(\tau) \geq 0, \quad \text { for any } p=1, \cdots, q, \quad \text { for any } \tau \in\left(0, \tilde{\mathcal{T}}_{2}\right) .
$$

On the other hand, since the mutual distances between the distinct clusters diverge towards infinity, and hence their mutual interactions energies tend to zero, one obtains, in view of the uniform convergence for each separate cluster, that

$$
\lim _{n \rightarrow+\infty} \tilde{\mathcal{F}}_{\text {att }}^{n}(\tau)=\sum_{p=1}^{q} F_{\text {att }}^{p}(\tau) \geq \sum_{p=1}^{q} F_{\text {att }}^{p}(0)=\lim _{n \rightarrow+\infty} \tilde{\mathcal{F}}_{\text {att }}^{n}(0), \quad \text { for } \tau \in\left(0, \tilde{\mathcal{T}}_{2}\right) .
$$


Therefore, for $n$ sufficiently large we are led to

$$
\tilde{\mathcal{F}}_{\text {att }}^{n}\left(\tilde{\mathcal{T}}_{2}\right)^{-\frac{1}{\omega}} \leq \tilde{\mathcal{F}}_{\text {att }}^{n}(0)^{-\frac{1}{\omega}}+\frac{1}{2 \kappa_{3}} .
$$

Scaling back to the original variables, this contradicts (7.31) and hence completes the proof.

From Proposition 7.2 and Proposition 7.3 we obtain

Proposition 7.4. There exists $\mathcal{K}_{2}>0$, depending only on $V$ and $M_{0}$, with the following properties. Assume that $J^{-}\left(s_{0}\right) \neq \emptyset$ and that $s \in \mathcal{I}_{0}^{\varepsilon}\left(s_{0}\right)$ satisfies

$$
d_{\min }^{\varepsilon, \mathrm{L}}(s) \geq \max \left(\beta_{0}, \beta_{1}\right) \varepsilon, \quad \text { and } \quad s+\mathcal{K}_{2} d_{\text {min }}^{\varepsilon,-}(s)^{\omega+2}<S .
$$

Then there exists some time $\mathcal{T}_{\text {col }}^{-}(s) \in \mathcal{I}_{0}^{\varepsilon}\left(s_{0}\right)$ such that

$$
\mathcal{T}_{\text {col }}^{\varepsilon,-}(s)-s \leq \mathcal{K}_{2} d_{\text {min }}^{\varepsilon,-}(s)^{\omega+2},
$$

and

$$
d_{\text {min }}^{\varepsilon, \mathrm{L}}\left(\mathcal{T}_{\text {col }}^{\varepsilon,-}(s)\right) \leq \max \left(\beta_{0}, 8 \mathfrak{q}_{1}\left(\alpha_{2}\right)\right) \varepsilon .
$$

Proof. We distinguish two cases.

Case I:

$$
d_{\min }^{\varepsilon, \mathrm{L}}(s)=d_{\min }^{\varepsilon,-}(s) \leq d_{\min }^{\varepsilon,+}(s) .
$$

In that case we will make use of Proposition 7.2 in an iterative argument. In view of (7.36), we are in position to invoke Proposition 7.2 at time $\hat{s}=s$ and set $s_{1}=\mathcal{T}_{1}^{\varepsilon}(s)$, so that in particular

$$
s_{1}-s \leq \mathcal{K}_{0} d_{\min }^{\varepsilon,-}(s)^{\omega+2} \quad \text { and } \quad d_{\min }^{\varepsilon,-}\left(s_{1}\right) \leq d_{\min }^{\varepsilon,+}\left(s_{1}\right) .
$$

Notice that by (7.36) and (7.40) we have $s_{1}<S$.

We distinguish two sub-cases:

Case I.1: $s_{1}=\mathcal{T}_{0}^{\varepsilon}\left(s_{0}\right)$ or $d_{\text {min }}^{\varepsilon,-}\left(s_{1}\right)<\beta_{0} \varepsilon$. In that case, we simply set $\mathcal{T}_{\text {col }}^{\varepsilon,-}(s)=s_{1}$ and we are done if we require $\mathcal{K}_{2} \geq \mathcal{K}_{2}$, by (7.40) and the definition of $\mathcal{T}_{0}^{\varepsilon}\left(s_{0}\right)$.

Case I.2: $s_{1}<\mathcal{T}_{0}^{\varepsilon}\left(s_{0}\right)$ and $d_{\text {min }}^{\varepsilon,-}\left(s_{1}\right) \geq \beta_{0} \varepsilon$. In that case, we may apply Proposition 7.2 at time $\hat{s}=s_{1}$ and set $s_{2}=\mathcal{T}_{1}^{\varepsilon}\left(s_{1}\right)$, so that in particular

$$
s_{2}-s_{1} \leq \mathcal{K}_{0} d_{\text {min }}^{\varepsilon,-}\left(s_{1}\right)^{\omega+2} \quad \text { and } \quad d_{\text {min }}^{\varepsilon,-}\left(s_{2}\right) \leq d_{\text {min }}^{\varepsilon,+}\left(s_{2}\right) .
$$

Moreover, since in that case $s_{1}=\mathcal{T}_{1}^{\varepsilon}(s)<\mathcal{T}_{0}^{\varepsilon}\left(s_{0}\right)$, it follows from (7.10) that

$$
d_{\min }^{\varepsilon,-}\left(s_{1}\right) \leq \frac{1}{10} d_{\min }^{\varepsilon,-}(s),
$$

and therefore from (7.41) we actually have

$$
s_{2}-s_{1} \leq \mathcal{K}_{0} 10^{-(\omega+2)} d_{\text {min }}^{\varepsilon,-}(s)^{\omega+2} \quad \text { and } \quad d_{\text {min }}^{\varepsilon,-}\left(s_{2}\right) \leq d_{\text {min }}^{\varepsilon,+}\left(s_{2}\right) .
$$


We then iterate the process until we fall into Case I.1. If we have not reached that stage up to step $m$, then thanks to Proposition 7.2 applied at time $\hat{s}=s_{m}$ we obtain, with $s_{m+1}:=\mathcal{T}_{1}^{\varepsilon}\left(s_{m}\right)$,

$$
s_{m+1}-s_{m} \leq \mathcal{K}_{0} d_{\min }^{\varepsilon,-}\left(s_{m}\right)^{\omega+2} \quad \text { and } \quad d_{\min }^{\varepsilon,-}\left(s_{m+1}\right) \leq d_{\min }^{\varepsilon,+}\left(s_{m+1}\right) .
$$

Moreover, since Case I.1 was not reached before step $m$, we have $s_{p}=\mathcal{T}_{1}^{\varepsilon}\left(s_{p-1}\right)<\mathcal{T}_{0}^{\varepsilon}\left(s_{0}\right)$ for all $p \leq m$, so that repeated use of (7.10) yields

$$
d_{\min }^{\varepsilon,-}\left(s_{p}\right) \leq\left(\frac{1}{10}\right)^{p} d_{\min }^{\varepsilon,-}(s), \quad \forall p \leq m .
$$

From (7.44) we thus also have

$$
s_{p+1}-s_{p} \leq \mathcal{K}_{0} 10^{-p(\omega+2)} d_{\text {min }}^{\varepsilon,-}(s)^{\omega+2}, \quad \forall p \leq m,
$$

and therefore by summation

$$
s_{m+1}-s \leq \mathcal{K}_{0}\left(\sum_{p=0}^{m} 10^{-p(\omega+2)}\right) d_{\min }^{\varepsilon,-}(s)^{\omega+2},
$$

so that in particular from (7.36) it holds $s_{m+1}<S$ if we choose $\mathcal{K}_{2} \geq 2 \mathcal{K}_{0}$. It follows from (7.45) that Case I.1 is necessarily reached in a finite number of steps, thus defining $\mathcal{T}_{\text {col }}^{\varepsilon,-}(s)$, and from (7.47) we obtain the upper bound

$$
\mathcal{T}_{\text {col }}^{\varepsilon,-}(s)-s \leq \mathcal{K}_{0}\left(\sum_{p=0}^{\infty} 10^{-p(\omega+2)}\right) d_{\text {min }}^{\varepsilon,-}(s)^{\omega+2} \leq 2 K_{0} d_{\text {min }}^{\varepsilon,-}(s)^{\omega+2},
$$

from which (7.37) follows.

Case II:

$$
d_{\min }^{\varepsilon, \mathrm{L}}(s)=d_{\min }^{\varepsilon,+}(s)<d_{\min }^{\varepsilon,-}(s) .
$$

Note that this implies that $J^{+}\left(s_{0}\right) \neq \emptyset$. We will show that Case II can be reduced to Case I after some controlled interval of time necessary for the repulsive forces to push $d_{\text {min }}^{\varepsilon,+}$ above $d_{\text {min }}^{\varepsilon,-}$. More precisely, we define the stopping time

$$
\mathcal{T}_{\text {cros }}^{\varepsilon}(s)=\inf \left\{\mathcal{T}_{0}^{\varepsilon}(s) \geq s^{\prime} \geq s, d_{\text {min }}^{\varepsilon,-}\left(s^{\prime}\right) \leq d_{\text {min }}^{\varepsilon,+}\left(s^{\prime}\right)\right\} .
$$

As in Case I, we implement an iterative argument, but based this time on Proposition 7.3. In view of (7.49) and (7.36), we may apply Proposition 7.3 at time $\hat{s}=s$ and set $s_{1}=\mathcal{T}_{2}^{\varepsilon}(s)$, so that in particular

$$
s_{1}-s \leq \mathcal{K}_{1} d_{\min }^{\varepsilon,+}(s)^{\omega+2} \leq \mathcal{K}_{1} d_{\min }^{\varepsilon,-}(s)^{\omega+2} .
$$

Notice that by (7.36) and (7.50) we have $s_{1}<S$ and therefore $d_{\min }^{\varepsilon,+}\left(s_{1}\right) \geq 10 d_{\min }^{\varepsilon,+}(s) \geq \beta_{1}$, and by (7.28)

$$
\begin{aligned}
\mathcal{F}_{\text {att }}^{\varepsilon}\left(s_{1}\right)^{-\frac{1}{\omega}} & \leq \mathcal{F}_{\text {att }}^{\varepsilon}(s)^{-\frac{1}{\omega}}+\frac{1}{\kappa_{3}} d_{\text {min }}^{\varepsilon,+}(s) \\
& \leq \mathcal{F}_{\text {att }}^{\varepsilon}(s)^{-\frac{1}{\omega}}+\frac{1}{10 \kappa_{3}} d_{\text {min }}^{\varepsilon,+}\left(s_{1}\right) .
\end{aligned}
$$


We distinguish two sub-cases.

Case II.1: $s_{1} \geq \mathcal{T}_{\text {cros }}^{\varepsilon}(s)$. In that case we proceed to Case I which we will apply starting at $s_{1}$ instead of $s$ and we set $\mathcal{T}_{\text {col }}^{\varepsilon,-}(s):=\mathcal{T}_{\text {col }}^{\varepsilon,-}\left(s_{1}\right)$. Since, combining the first inequality of (7.51) with (7.7), we deduce that

$$
d_{\min }^{\varepsilon,-}\left(s_{1}\right) \leq \kappa_{3} \kappa_{2}^{-1} d_{\min }^{\varepsilon,-}(s)+d_{\min }^{\varepsilon,+}(s) \leq\left(\kappa_{3} \kappa_{2}^{-1}+1\right) d_{\min }^{\varepsilon,-}(s),
$$

the equivalent of (7.48) becomes

$$
\begin{aligned}
\mathcal{T}_{\text {col }}^{\varepsilon,-}\left(s_{1}\right)-s_{1} & \leq \mathcal{K}_{0}\left(\sum_{p=0}^{\infty} 10^{-p(\omega+2)}\right) d_{\text {min }}^{\varepsilon,-}\left(s_{1}\right)^{\omega+2} \\
& \leq 2 \mathcal{K}_{0} d_{\text {min }}^{\varepsilon,-}\left(s_{1}\right)^{\omega+2} \\
& \leq 2 \mathcal{K}_{0}\left(\kappa_{3} \kappa_{2}^{-1}+1\right)^{\omega+2} d_{\text {min }}^{\varepsilon,-}(s)^{\omega+2},
\end{aligned}
$$

and therefore it follows from (7.50) that

$$
\mathcal{T}_{\text {col }}^{\varepsilon,-}(s)-s \leq \mathcal{T}_{\text {col }}^{\varepsilon,-}\left(s_{1}\right)-s_{1}+\left(s_{1}-s\right) \leq\left(\mathcal{K}_{1}+2 \mathcal{K}_{0}\left(\kappa_{3} \kappa_{2}^{-1}+1\right)^{\omega+2}\right) d_{\text {min }}^{\varepsilon,-}(s)^{\omega+2},
$$

and (7.37) follows if $\mathcal{K}_{2} \geq \mathcal{K}_{1}+2 \mathcal{K}_{0}\left(\kappa_{3} \kappa_{2}^{-1}+1\right)^{\omega+2}$.

Case II.2: $s_{1}<\mathcal{T}_{\text {cros }}^{\varepsilon}(s)$. In that case we proceed to construct $s_{2}=\mathcal{T}_{2}^{\varepsilon}\left(s_{1}\right)$. Notice that combining the second inequality of (7.51) with (7.7), we deduce that

$$
d_{\min }^{\varepsilon,-}\left(s_{1}\right) \leq \kappa_{3} \kappa_{2}^{-1} d_{\min }^{\varepsilon,-}(s)+\frac{1}{5} d_{\min }^{\varepsilon,+}\left(s_{1}\right) \leq \kappa_{3} \kappa_{2}^{-1} d_{\min }^{\varepsilon,-}(s)+\frac{1}{5} d_{\min }^{\varepsilon,-}\left(s_{1}\right),
$$

so that

$$
d_{\min }^{\varepsilon,+}\left(s_{1}\right) \leq d_{\min }^{\varepsilon,-}\left(s_{1}\right) \leq \frac{5}{4} \kappa_{3} \kappa_{2}^{-1} d_{\min }^{\varepsilon,-}(s) .
$$

We explain now the iterative argument. Assume that for some $m \geq 1$ have already constructed $s_{1}, \cdots, s_{m}$, such that for $2 \leq p \leq m$

$$
s_{p}<S, \quad \beta_{1} \varepsilon \leq d_{\min }^{\varepsilon,+}\left(s_{p}\right) \leq d_{\min }^{\varepsilon,-}\left(s_{p}\right), \quad s_{p}=\mathcal{T}_{2}^{\varepsilon}\left(s_{p-1}\right) .
$$

First, repeated use of (7.23) yields

$$
d_{\min }^{\varepsilon,+}\left(s_{p}\right) \geq 10^{p} d_{\min }^{\varepsilon,+}(s), \quad \forall 1 \leq p \leq m,
$$

and actually

$$
d_{\text {min }}^{\varepsilon,+}\left(s_{p}\right) \geq 10^{p-q} d_{\text {min }}^{\varepsilon,+}(s), \quad \forall 1 \leq q \leq p \leq m .
$$

Hence, by repeated use of (7.28), we obtain

$$
\begin{aligned}
\mathcal{F}_{\text {att }}^{\varepsilon}\left(s_{m}\right)^{-\frac{1}{\omega}} & \leq \mathcal{F}_{\text {att }}^{\varepsilon}(s)^{-\frac{1}{\omega}}+\frac{1}{\kappa_{3}}\left(d_{\text {min }}^{\varepsilon,+}(s)+\sum_{p=1}^{m-1} d_{\text {min }}^{\varepsilon,+}\left(s_{p}\right)\right) \\
& \leq \mathcal{F}_{\text {att }}^{\varepsilon}(s)^{-\frac{1}{\omega}}+\frac{1}{\kappa_{3}} \sum_{p=0}^{m-1} 10^{-p} d_{\min }^{\varepsilon,+}\left(s_{m-1}\right) \\
& \leq \mathcal{F}_{\text {att }}^{\varepsilon}(s)^{-\frac{1}{\omega}}+\frac{2}{\kappa_{3}} d_{\text {min }}^{\varepsilon,+}\left(s_{m-1}\right) \\
& \leq \mathcal{F}_{\text {att }}^{\varepsilon}(s)^{-\frac{1}{\omega}}+\frac{1}{5 \kappa_{3}} d_{\text {min }}^{\varepsilon,+}\left(s_{m}\right) .
\end{aligned}
$$


Combining the latter with (7.7), we deduce that

$$
d_{\min }^{\varepsilon,-}\left(s_{m}\right) \leq \kappa_{3} \kappa_{2}^{-1} d_{\min }^{\varepsilon,-}(s)+\frac{1}{5} d_{\min }^{\varepsilon,+}\left(s_{m}\right) \leq \kappa_{3} \kappa_{2}^{-1} d_{\min }^{\varepsilon,-}(s)+\frac{1}{5} d_{\min }^{\varepsilon,-}\left(s_{m}\right),
$$

so that

$$
d_{\min }^{\varepsilon,+}\left(s_{m}\right) \leq d_{\min }^{\varepsilon,-}\left(s_{m}\right) \leq \frac{5}{4} \kappa_{3} \kappa_{2}^{-1} d_{\min }^{\varepsilon,-}(s) .
$$

Let $s_{m+1}:=\mathcal{T}_{2}^{\varepsilon}\left(s_{m}\right)$. Then by $(7.26)$ and $(7.57)$

$$
\begin{aligned}
s_{m+1}-s & \leq \mathcal{K}_{1}\left(d_{\min }^{\varepsilon,+}(s)^{\omega+2}+\sum_{p=1}^{m} d_{\min }^{\varepsilon,+}\left(s_{p}\right)^{\omega+2}\right) \\
& \leq \mathcal{K}_{1} \sum_{p=0}^{m-1} 10^{-(\omega+2)(m-p)} d_{\min }^{\varepsilon,+}\left(s_{m}\right)^{\omega+2} \\
& \leq 2 \mathcal{K}_{1} d_{\min }^{\varepsilon,+}\left(s_{m}\right)^{\omega+2} .
\end{aligned}
$$

Combining (7.60) with (7.59) we are led to

$$
s_{m+1}-s \leq 2\left(\frac{5 \kappa_{3}}{4 \kappa_{2}}\right)^{\omega+2} \mathcal{K}_{1} d_{\min }^{\varepsilon,-}(s)^{\omega+2}
$$

and therefore by (7.36) we have $s_{m+1}<S$.

Combining (7.59) with (7.57), we obtain

$$
0 \leq d_{\min }^{\varepsilon,-}\left(s_{m}\right)-d_{\min }^{\varepsilon,+}\left(s_{m}\right) \leq \frac{\kappa_{3}}{\kappa_{2}} d_{\min }^{\varepsilon,-}(s)-10^{m}\left(d_{\min }^{\varepsilon,+}(s)\right),
$$

and therefore necessarily

$$
m \leq \log _{10}\left(\frac{\kappa_{3} d_{\min }^{\varepsilon,-}(s)}{\kappa_{2} d_{\min }^{\varepsilon,+}(s)}\right) .
$$

It follows that the number $m_{0}=\sup \left\{m \in \mathbb{N}_{*}, d_{\text {min }}^{\varepsilon,-}\left(s_{m}\right) \geq d_{\min }^{\varepsilon,+}\left(s_{m}\right)\right\}$ is finite, and at that stage we proceed to Case I as in Case II.1 above, and the conclusion follows likewise, replacing (7.52) by

$$
d_{\min }^{\varepsilon,+}\left(s_{m_{0}+1}\right) \leq \frac{9}{4} \kappa_{3} \kappa_{2}^{-1} d_{\min }^{\varepsilon,-}(s)
$$

which is obtained combining

$$
d_{\min }^{\varepsilon,+}\left(s_{m_{0}+1}\right) \leq \kappa_{3} \kappa_{2}^{-1} d_{\min }^{\varepsilon,-}(s)+d_{\min }^{\varepsilon,+}\left(s_{m_{0}}\right),
$$

with

$$
d_{\min }^{\varepsilon,+}\left(s_{m_{0}}\right) \leq d_{\min }^{\varepsilon,-}\left(s_{m_{0}}\right) \leq \frac{5}{4} \kappa_{3} \kappa_{2}^{-1} d_{\min }^{\varepsilon,-}(s) .
$$




\subsection{Proof of Proposition 7.1}

We will fix the value of the constants $\kappa_{1}, \alpha_{3}$ and $\mathcal{K}_{\text {col }}$ in the course of the proof. Let $s_{0}$ be as in the statement. We first require that

$$
\alpha_{3} \geq \alpha_{2} \quad \text { and that } \quad \alpha_{3} \geq 16 \mathfrak{q}_{1}\left(\alpha_{2}\right),
$$

so that assumption $\mathcal{W} \mathcal{P}_{\varepsilon}^{\mathrm{L}}\left(\alpha_{3}, s_{0}\right)$ implies assumption 7.8 of Subsection 7.1.

Next, we set $s=s_{0}+\varepsilon^{\omega+2}$ and we wish to make sure that the assumptions of Proposition 7.4 are satisfied at time $s$. In view of the upper bound (7) on the velocity of the front set, we deduce that

$$
d_{\min }^{\varepsilon, \mathrm{L}}(s) \geq d_{\min }^{\varepsilon, \mathrm{L}}\left(s_{0}\right)-C \rho_{0}^{\frac{1}{\omega+2}} \varepsilon \geq \alpha_{3} \varepsilon-C \rho_{0}^{\frac{1}{\omega+2}} \varepsilon \geq \max \left(\beta_{0}, \beta_{1}\right) \varepsilon
$$

provided we choose $\alpha_{3}$ sufficiently large. Also,

$$
\frac{1}{2} d_{\min }^{\varepsilon,-}\left(s_{0}\right) \leq d_{\min }^{\varepsilon, \mathrm{L}}\left(s_{0}\right)-C \rho_{0}^{\frac{1}{\omega+2}} \varepsilon \leq d_{\min }^{\varepsilon, \mathrm{L}}(s) \leq d_{\min }^{\varepsilon, \mathrm{L}}\left(s_{0}\right)+C \rho_{0}^{\frac{1}{\omega+2}} \varepsilon \leq 2 d_{\min }^{\varepsilon, \mathrm{L}}\left(s_{0}\right),
$$

and therefore provided we choose

$$
\mathcal{K}_{\text {col }} \geq 2^{\omega+2} \mathcal{K}_{2}
$$

it follows from the assumption $s_{0}+\mathcal{K}_{\text {col }} d_{\text {min }}^{\varepsilon, \mathrm{L}}\left(s_{0}\right)^{\omega+2}<S$ that $s+\mathcal{K}_{2} d_{\min }^{\varepsilon, \mathrm{L}}(s)^{\omega+2}<S$. Therefore we may apply Proposition 7.4. Let $\mathcal{T}_{\text {col }}^{\varepsilon,-}(s) \in \mathcal{I}_{0}^{\varepsilon}\left(s_{0}\right)$ be given by its statement, so that by $(7.61)$

$$
\mathcal{T}_{\text {col }}^{\varepsilon,-}(s)-s \leq 2^{\omega+2} \mathcal{K}_{2} d_{\text {min }}^{\varepsilon,-}\left(s_{0}\right)^{\omega+2},
$$

and

$$
d_{\text {min }}^{\varepsilon, \mathrm{L}}\left(\mathcal{T}_{\text {col }}^{\varepsilon,-}(s)\right) \leq \max \left(\beta_{0}, 8 \mathfrak{q}_{1}\left(\alpha_{2}\right)\right) \varepsilon .
$$

By Proposition 3.1, there exists some time $\mathcal{T}_{\text {col }}^{\varepsilon,+}\left(s_{0}\right) \in\left[\mathcal{T}_{\text {col }}^{\varepsilon,-}(s), \mathcal{T}_{\text {col }}^{\varepsilon,-}(s)+\mathfrak{q}_{0}\left(\alpha_{3}\right) \varepsilon^{\omega+2}\right]$ such that $\operatorname{WPI}_{\varepsilon}^{\mathrm{L}}\left(\alpha_{3} \varepsilon, \mathcal{T}_{\text {col }}^{\varepsilon,+}\left(s_{0}\right)\right)$ holds. In view of $(7.61)$ and since $d_{\text {min }}^{\varepsilon,-}\left(s_{0}\right) \geq \alpha_{3} \varepsilon$, it follows that

$$
\begin{aligned}
0 \leq \mathcal{T}_{\text {col }}^{\varepsilon,+}\left(s_{0}\right)-s_{0} & \leq \varepsilon^{\omega+2}+2^{\omega+2} \mathcal{K}_{2} d_{\text {min }}^{\varepsilon,-}\left(s_{0}\right)^{\omega+2}+\mathfrak{q}_{0}\left(\alpha_{3}\right) \varepsilon^{\omega+2} \\
& \leq\left(2^{\omega+2} \mathcal{K}_{2}+\frac{1+\mathfrak{q}_{0}\left(\alpha_{3}\right)}{\alpha_{3}^{\omega+2}}\right) d_{\text {min }}^{\varepsilon,-}\left(s_{0}\right)^{\omega+2} \\
& \leq \mathcal{K}_{\text {col }} d_{\text {min }}^{\varepsilon,-}\left(s_{0}\right)^{\omega+2}
\end{aligned}
$$

provided we finally fix the value of $\mathcal{K}_{\text {col }}$ as

$$
\mathcal{K}_{\text {col }}=\left(2^{\omega+2} \mathcal{K}_{2}+\frac{1+\mathfrak{q}_{0}\left(\alpha_{3}\right)}{\alpha_{3}^{\omega+2}}\right)
$$

[Note that at this stage $\mathcal{K}_{\text {col }}$ is fixed but its definition depend on $\alpha_{3}$ which has not yet been fixed. Of course when we will fix $\alpha_{3}$ below we shall do it without any reference to $\mathcal{K}_{\text {col }}$, in order to avoid impossible loops]

Next, we first claim that

$$
\mathfrak{E}_{\varepsilon}^{\mathrm{L}}\left(s_{0}\right) \geq \mathfrak{E}_{\varepsilon}^{\mathrm{L}}(s) \geq \mathfrak{E}_{\varepsilon}^{\mathrm{L}}\left(\mathcal{T}_{\text {col }}^{\varepsilon,+}\left(s_{0}\right)\right) .
$$


In view of Corollary 3.2, it suffices to check that $\mathrm{L} \geq \mathrm{L}_{0}\left(s_{0}, \mathcal{T}_{\text {col }}^{\varepsilon,+}\left(s_{0}\right)\right)$, where we recall that the function $\mathrm{L}_{0}(\cdot)$ was defined in (3.6). In view of (7.63), this reduces to

$$
100 \mathrm{C}_{e} \mathrm{~L}^{-(\omega+2)} \mathcal{K}_{\text {col }} d_{\text {min }}^{\varepsilon,-}\left(s_{0}\right)^{\omega+2} \leq \frac{\mu_{1}}{4} .
$$

Since by $(7.2)$ we have $d_{\min }^{\varepsilon,-}\left(s_{0}\right) \leq 2 \kappa_{0}\left(s_{0}\right) \mathrm{L}$, it suffices therefore that

$$
\kappa_{0}\left(s_{0}\right) \leq \frac{1}{2}\left(\frac{\mu_{1}}{400 \mathrm{C}_{\mathrm{e}} \mathcal{K}_{\mathrm{col}}}\right)^{\frac{1}{\omega+2}} \equiv \mathrm{\kappa}_{1},
$$

and we have now fixed the value of $\mathrm{k}_{1}$.

Next, we claim that actually

$$
\mathfrak{E}_{\varepsilon}^{\mathrm{L}}\left(\mathcal{T}_{\text {col }}^{\varepsilon,+}\left(s_{0}\right)\right) \leq \mathfrak{E}_{\varepsilon}^{\mathrm{L}}\left(s_{0}\right)-\mu_{1}
$$

Indeed, otherwise by Corollary 3.2 we would have $\mathfrak{E}_{\varepsilon}^{\mathrm{L}}\left(\mathcal{T}_{\text {col }}^{\varepsilon,+}\left(s_{0}\right)\right)=\mathfrak{E}_{\varepsilon}^{\mathrm{L}}\left(s_{0}\right)$, and therefore condition $\mathcal{C}\left(\alpha_{3} \varepsilon, \mathrm{L}, s_{0}, \mathcal{T}_{\text {col }}^{\varepsilon,+}\left(s_{0}\right)\right)$ of Subsection 3.4 would hold. Invoking Proposition 3.3, this would imply that condition $\mathcal{W} P^{\mathrm{L}}\left(\Lambda_{\log }\left(\alpha_{3} \varepsilon\right), \tau\right)$ holds for $\tau \in\left(s_{0}+\varepsilon^{\omega+2}, \mathcal{T}_{\text {col }}^{\varepsilon,+}\left(s_{0}\right)\right)$, so that in particular

$$
d_{\text {min }}^{\varepsilon}\left(\mathcal{T}_{\text {col }}^{\varepsilon,-}\left(s_{0}\right)\right) \geq \Lambda_{\log }\left(\alpha_{3} \varepsilon\right) .
$$

It suffices thus to choose $\alpha_{3}$ sufficiently big so that

$$
\left.\Lambda_{\log }\left(\alpha_{3} \varepsilon\right)>\max \left(\beta_{0}, 8 \mathfrak{q}_{1}\left(\alpha_{2}\right)\right)\right) \varepsilon,
$$

and the contradiction then follows from (7.62).

\subsection{Proof of Proposition 7}

We will fix the values of $\kappa_{*}$ and $\rho_{*}$ in the course of the proofs, as the smallest numbers which satisfy a finite number lower bound inequalities.

First, recall that it follows from (53) and (7) that if $0 \leq s \leq \rho_{0}(R-r)^{\omega+2}$ then

$$
\mathfrak{D}_{\varepsilon}(s) \cap \mathrm{I}_{4 \mathrm{~L}} \subset \underset{k \in J_{0}}{\cup}\left(b_{k}^{\varepsilon}-R, b_{k}^{\varepsilon}+R\right) \subset \mathrm{I}_{2 \kappa_{0} \mathrm{~L}} \text {, where the union is disjoint, }
$$

and in particular $\mathcal{C}_{\mathrm{L}, S}$ holds where

$$
S:=\rho_{0}(R-r)^{\omega+2} \geq \rho_{0}\left(\frac{R}{2}\right)^{\omega+2} .
$$

Having (3.6) in mind, and in view of (7.64) and (54), we estimate

$$
100 \mathrm{C}_{\mathrm{e}} \mathrm{L}^{-(\omega+2)} S \leq 100 \mathrm{C}_{\mathrm{e}}\left(\frac{R}{2 \mathrm{~L}}\right)^{\omega+2} S \leq 100 \mathrm{C}_{\mathrm{e}} \alpha_{*}^{-(\omega+2)} \leq \frac{\mu_{1}}{4},
$$

where the last inequality follows provided we choose $\alpha_{*}$ sufficiently large. As a consequence, the function $\mathfrak{E}_{\varepsilon}^{\mathrm{L}}$ is non-increasing on the set of times $s$ in the interval $\left[\varepsilon^{\omega} \mathrm{L}^{2}, S\right]$ where $\mathcal{W P}_{\varepsilon}^{\mathrm{L}}\left(\alpha_{1} \varepsilon, s\right)$ holds. 
For such times $s$, the front points $\left\{a_{k}^{\varepsilon}(s)\right\}_{k \in J(s)}$ are well-defined, and for $q \in J_{0}$, we have defined in the introduction $J_{q}(s)=\left\{k \in J(s), a_{k}^{\varepsilon}(s) \in\left[b_{q}^{\varepsilon}-R, b_{q}^{\varepsilon}+R\right]\right\}$, and we have set $\ell_{q}=\sharp J_{q}$ and $J_{q}(s)=\left\{k_{q}, k_{q+1}, \cdots, k_{q+\ell_{q}-1}\right\}$, where $k_{1}=1$, and $k_{q}=\ell_{1}+\cdots+\ell_{q-1}+1$, for $q \geq 2$.

Step 1. Annihilations of all the pairs of fronts-antifronts. We claim that there exists some time $\tilde{s} \in\left(\varepsilon^{\omega} \mathrm{L}^{2}, \frac{1}{2} S\right)$ such that $\mathcal{W} \mathcal{P}_{\varepsilon}^{\mathrm{L}}\left(\delta_{\log }^{\varepsilon}, \tilde{s}\right)$ holds and such that for any $q \in J_{0}, \epsilon_{k+\frac{1}{2}}(\tilde{s})=+1$, for $\quad k \in J_{q}(\tilde{s}) \backslash\left\{k_{q}(\tilde{s})+\ell_{q}(\tilde{s})-1\right\}$ or $\sharp J_{q}(\tilde{s}) \leq 1$, or equivalently that $\dagger_{k}(\tilde{s})=\dagger_{k^{\prime}}(\tilde{s})$ for $k$ and $k^{\prime}$ in the same $J_{q}(\tilde{s})$. In particular, $d_{\min }^{\varepsilon,-}(\tilde{s}) \geq 2 R$.

Proof of the claim. If we require $\alpha_{*}$ to be sufficiently large, then by (54) we have that $\varepsilon^{\omega} \mathrm{L}^{2}+$ $\varepsilon^{\omega+1} \mathrm{~L} \leq S / 2$, and therefore by Proposition 3 we may choose a first time

$$
s_{0} \in\left[\varepsilon^{\omega} \mathrm{L}^{2}, \varepsilon^{\omega} \mathrm{L}^{2}+\varepsilon^{\omega+1} \mathrm{~L}\right] \text { such that } \mathcal{W P}_{\varepsilon}^{\mathrm{L}}\left(\delta_{\log }^{\varepsilon}, s_{0}\right) \text { holds. }
$$

Actually, we have

$$
s_{0} \leq \varepsilon^{\omega} \mathrm{L}^{2}+\varepsilon^{\omega+1} \mathrm{~L} \leq 2 \varepsilon^{\omega} \mathrm{L}^{2} \leq 2 \alpha_{*}^{-(\omega+2)} r^{\omega+2} \leq 2 \alpha_{*}^{-2(\omega+2)} R^{\omega+2} \leq \frac{1}{\rho_{0}} 2^{\omega+3} \alpha_{*}^{-2(\omega+2)} S .
$$

Note that by (7) we have the inclusion

$$
\mathfrak{D}_{\varepsilon}\left(s_{0}\right) \cap \mathrm{I}_{\mathrm{L}} \subset \mathcal{N}\left(b, r_{0}\right),
$$

where

$$
r_{0}=r+\left(\frac{s_{0}}{\rho_{0}}\right)^{\frac{1}{\omega+2}} \leq 2 r,
$$

provided once more that $\alpha_{*}$ is sufficiently large, and where for $\rho>0$ we have set $\mathcal{N}(b, \rho)=$ $\cup_{q \in J_{0}}\left[b_{j}^{\varepsilon}-\rho, b_{j}^{\varepsilon}+\rho\right]$. In view of the confinement condition (53) only two cases can occur:

$$
\text { i) } \quad d_{\min }^{\varepsilon,-}\left(s_{0}\right) \geq 3 R-2 r_{0} \quad \text { or } \quad \text { ii) } \quad d_{\min }^{\varepsilon,-}\left(s_{0}\right) \leq 2 r_{0} .
$$

If case i) occurs, then, for any $q \in J_{0}$, we have $\epsilon_{k+\frac{1}{2}}=+1$, for any $k \in J_{q}\left(\tau_{1}\right) \backslash\left\{k_{q}\left(s_{0}\right)+\right.$ $\left.\ell_{q}\left(s_{0}\right)-1\right\}$. Choosing $\tilde{s}=s_{0}$, Step 1 is completed in the case considered.

If instead case ii) occurs, then we will make use of Proposition 7.1 to remove the small pairs of fronts-antifronts present at small scales. More precisely, assume that for some $j \geq 0$ we have constructed $0 \leq s_{j} \leq S$ and $r_{j}>0$ such that $\mathcal{W} \mathcal{P}_{\varepsilon}^{\mathrm{L}}\left(\delta_{\log }^{\varepsilon}, s_{j}\right)$ holds, such that we have

$$
\mathfrak{D}_{\varepsilon}\left(s_{j}\right) \cap \mathrm{I}_{\mathrm{L}} \subset \mathcal{N}\left(b, r_{j}\right), \quad \mathfrak{E}_{\varepsilon}^{\mathrm{L}}\left(s_{j}\right) \leq \mathfrak{E}_{\varepsilon}^{\mathrm{L}}\left(s_{0}\right)-j \mu_{1},
$$

as well as the estimates,

$$
r_{0} \leq r_{j} \leq \gamma^{j} r_{0} \leq \frac{R}{2}, \quad s_{j} \leq s_{0}+\left(2^{\omega+2} \mathcal{K}_{\mathrm{col}}+1\right) \frac{\gamma^{j(\omega+2)}-1}{\gamma^{\omega+2}-1} r_{0}^{\omega+2} \leq \frac{S}{2},
$$

where $\gamma:=\left(2+2\left(\frac{\mathcal{K}_{\mathrm{col}}}{\rho_{0}}\right)^{\frac{1}{\omega+2}}\right)$, and moreover that case ii) holds at step $j$, that is

$$
d_{\min }^{\varepsilon,-}\left(s_{j}\right) \leq 2 r_{j} \leq R
$$


Let $\tilde{s}_{j}:=\mathcal{T}_{\text {col }}^{\varepsilon,+}\left(s_{j}\right)$ be given by Proposition 7.1 (the confinement condition holds in view of (7.64) and we have $\delta_{\log }^{\varepsilon} \geq \alpha_{3} \varepsilon$ provided $\alpha_{*}$ is sufficiently large), and let then $s_{j+1} \in$ $\left[\tilde{s}_{j}, \tilde{s}_{j}+\varepsilon^{\omega+1} \mathrm{~L}\right]$ satisfying $\mathcal{W} \mathcal{P}_{\varepsilon}^{\mathrm{L}}\left(\delta_{\log }^{\varepsilon}, s_{j+1}\right)$ be given by Proposition 3 . In particular, we have

$$
\mathfrak{E}_{\varepsilon}^{\mathrm{L}}\left(s_{j+1}\right) \leq \mathfrak{E}_{\varepsilon}^{\mathrm{L}}\left(\tilde{s}_{j}\right) \leq \mathfrak{E}_{\varepsilon}^{\mathrm{L}}\left(s_{j}\right)-\mu_{1} \leq \mathfrak{E}_{\varepsilon}^{\mathrm{L}}\left(s_{0}\right)-(j+1) \mu_{1} .
$$

Since

$$
s_{j+1}-s_{j} \leq \mathcal{K}_{\text {col }}\left(2 r_{j}\right)^{\omega+2}+\varepsilon^{\omega+1} \mathrm{~L} \leq\left(2^{\omega+2} \mathcal{K}_{\text {col }}+1\right) r_{j}^{\omega+2},
$$

we have, in view of $(7.67)$

$$
\begin{aligned}
s_{j+1} & \leq s_{0}+\left(2^{\omega+2} \mathcal{K}_{\mathrm{col}}+1\right)\left[\frac{\gamma^{j(\omega+2)}-1}{\gamma^{\omega+2}-1}+\gamma^{j(\omega+2)}\right] r_{0}^{\omega+2} \\
& \leq s_{0}+\left(2^{\omega+2} \mathcal{K}_{\mathrm{col}}+1\right) \frac{\gamma^{(j+1)(\omega+2)}-1}{\gamma^{\omega+2}-1} r_{0}^{\omega+2}
\end{aligned}
$$

and by $(7) \mathfrak{D}_{\varepsilon}\left(s_{j+1}\right) \cap \mathrm{I}_{\mathrm{L}} \subset \mathcal{N}\left(b, r_{j+1}\right)$, where

$$
r_{j+1}=r_{j}+2\left(\frac{\mathcal{K}_{\mathrm{col}}}{\rho_{0}}\right)^{\frac{1}{\omega+2}} r_{j}+\frac{1}{\rho_{0}} \varepsilon\left(\frac{\mathrm{L}}{\varepsilon}\right)^{\frac{1}{\omega+2}} \leq\left(2+2\left(\frac{\mathcal{K}_{\mathrm{col}}}{\rho_{0}}\right)^{\frac{1}{\omega+2}}\right) r_{j}=\gamma r_{j} .
$$

In view of (7.65) and (54), we also have

$$
\gamma^{j+1} r_{0} \leq 2 \gamma^{j+1} \alpha_{*}^{-1} R
$$

and

$$
\begin{aligned}
& s_{0}+\left(2^{\omega+2} \mathcal{K}_{\mathrm{col}}+1\right) \frac{\gamma^{(j+1)(\omega+2)}-1}{\gamma^{\omega+2}-1} r_{0}^{\omega+2} \\
\leq & {\left[\frac{2^{\omega+3}}{\rho_{0}} \alpha_{*}^{-(\omega+2)}+\frac{2^{2 \omega+4}}{\rho_{0}}\left(2^{\omega+2} \mathcal{K}_{\mathrm{col}}+1\right) \frac{\gamma^{(j+1)(\omega+2)}-1}{\gamma^{\omega+2}-1}\right] \alpha_{*}^{-(\omega+2)} S . }
\end{aligned}
$$

It follows from (7.70), (7.71), (7.72) and (7.73) that if $\alpha_{*}$ is sufficiently large (depending only on $M_{0}, V$ and $j$ ), then (7.67) holds also for $s_{j+1}$. As above we distinguish two cases:

$$
\text { i) } \quad d_{\min }^{\varepsilon,-}\left(s_{j+1}\right) \geq 3 R-2 r_{j+1} \quad \text { or } \quad \text { ii) } \quad d_{\min }^{\varepsilon,-}\left(s_{j+1}\right) \leq 2 r_{j+1} .
$$

If case i) holds then by (7.67) we have $d_{\min }^{\varepsilon,-}\left(s_{j+1}\right) \geq 2 R$, we set $\tilde{s}=s_{j+1}$ which therefore satisfies the requirements of the claim, and we proceed to Step 2.

If case ii) occur then we proceed to construct $s_{j+2}$ as above. The key fact in this recurrence construction is the second inequality in (7.66), which, since $\mathfrak{E}_{\varepsilon}^{\mathrm{L}}\left(s_{j}\right) \geq 0$ independently of $j$, implies that the process as to reach case i) in a number of steps less than or equal to $M_{0} / \mu_{1}$. In particular, choosing the constant $\alpha_{*}$ sufficiently big so that the right-hand side of (7.72) is smaller than $R / 2$ for all $0 \leq j \leq M_{0} / \mu_{1}$ and so that the right hand side of (7.73) is smaller than $S / 2$ for all $0 \leq j \leq M_{0} / \mu_{1}$ ensures that the construction was licit and that the process necessarily reaches case i) before it could reach $j=M_{0} / \mu_{1}+1$, so defining $\tilde{s}$ as above. 
Step 2: Divergence of the remaining repulsing fronts at small scale. At this stage we have constructing $\tilde{s} \in\left[\varepsilon^{\omega} L^{2}, \frac{1}{2} S\right]$ which satisfies the requirements of the claim in Step 1. Moreover, note that in view of (7.65) and (7.67) we have the upper bound

$$
\tilde{s} \leq\left(2 \alpha_{*}^{-(\omega+2)}+2^{\omega+2}\left(2^{\omega+2} \mathcal{K}_{\mathrm{col}}+1\right) \frac{\gamma^{\frac{M_{0}}{\mu_{1}}(\omega+2)}-1}{\gamma^{\omega+2}-1}\right) r^{\omega+2} .
$$

In order to complete the proof, we next distinguish two cases:

$$
\text { i) } \sharp J_{q}(\tilde{s}) \leq 1 \text {, for any } q \in J_{0} . \quad \text { ii) } \sharp J_{q_{0}}(\tilde{s})>1 \text {, form some } q_{0} \in J_{0} \text {. }
$$

If case i) holds, then we actually have

$$
d_{\min }^{\varepsilon, \mathrm{L}}(\tilde{s}) \geq 2 R
$$

Since $2 R \geq 16 \mathfrak{q}_{1}\left(\delta_{\log }^{\varepsilon}\right) \varepsilon$ when $\alpha_{*}$ is sufficiently large, it follows from Corollary 3.3 that $\mathcal{W P}_{\varepsilon}^{\mathrm{L}}\left(\delta_{\log \log }^{\varepsilon}, s\right)$ holds for any $\tilde{s}+\varepsilon^{2+\omega} \leq s \leq \mathcal{T}_{0}^{\varepsilon}\left(\delta_{\log }^{\varepsilon}, \tilde{s}\right)$, where

$$
\mathcal{T}_{0}^{\varepsilon}\left(\delta_{\log }^{\varepsilon}, \tilde{s}\right)=\max \left\{\tilde{s}+\varepsilon^{2+\omega} \leq s \leq S \quad \text { s.t. } \quad d_{\min }^{\varepsilon, \mathrm{L}}\left(s^{\prime}\right) \geq 8 \mathfrak{q}_{1}\left(\delta_{\log }^{\varepsilon}\right) \varepsilon \quad \forall s^{\prime} \in\left[\tilde{s}+\varepsilon^{\omega+2}, s\right]\right\} .
$$

In particular, $\mathcal{W} \mathcal{P}_{\varepsilon}^{\mathrm{L}}\left(\delta_{\log \log }^{\varepsilon}, s\right)$ holds for any $s$ in $\tilde{s}+\varepsilon^{2+\omega} \leq s \leq \mathcal{T}_{3}^{\varepsilon}\left(\delta_{\log }^{\varepsilon}, \tilde{s}\right)$, where

$$
\mathcal{T}_{3}^{\varepsilon}\left(\delta_{\log }^{\varepsilon}, \tilde{s}\right)=\max \left\{\tilde{s}+\varepsilon^{2+\omega} \leq s \leq S \quad \text { s.t. } \quad d_{\min }^{\varepsilon, \mathrm{L}}\left(s^{\prime}\right) \geq R \quad \forall s^{\prime} \in\left[\tilde{s}+\varepsilon^{\omega+2}, s\right]\right\} .
$$

In view of (7.75) and estimate (7), we obtain the lower bound

$$
\mathcal{T}_{3}^{\varepsilon}\left(\delta_{\log }^{\varepsilon}, \tilde{s}\right) \geq \tilde{s}+\rho_{0} R^{\omega+2} .
$$

Note that (7.76) and (54) also yield

$$
\mathcal{T}_{3}^{\varepsilon}\left(\delta_{\log }^{\varepsilon}, \tilde{s}\right) \geq \tilde{s}+\rho_{0} \alpha_{*}^{-1} r^{\omega+2} \geq \rho_{0} \alpha_{*}^{-1} r^{\omega+2} .
$$

Combining (7.74) and (7.77) we deduce in particular that

$$
\mathcal{W P}_{\varepsilon}^{\mathrm{L}}\left(\alpha_{1} \varepsilon, s_{r}\right) \text { holds } \quad \text { and } \quad d_{\min }^{\varepsilon, \mathrm{L}}\left(s_{r}\right) \geq R \geq r,
$$

which is the claim of Proposition 7, provided

$$
\rho_{*} \geq\left(3+2^{\omega+2}\left(2^{\omega+2} \mathcal{K}_{\mathrm{col}}+1\right) \frac{\gamma^{\frac{M_{0}}{\mu_{1}}(\omega+2)}-1}{\gamma^{\omega+2}-1}\right) \quad \text { and } \quad \rho_{*} \leq \rho_{0} \alpha_{*}^{-1} .
$$

It remains to consider the situation where case ii) holds. In that case, we have

$$
d_{\min }^{\varepsilon,-}(\tilde{s}) \geq 2 R \quad \text { and } \quad d_{\min }^{\varepsilon,+}(\tilde{s}) \leq 2 \gamma^{M_{0} / \mu_{1}} r \leq R,
$$

so that we are in a situation suited for Proposition 7.3. We may actually apply Proposition 7.3 recursively with $s_{0}:=\tilde{s}$ and $\hat{s} \equiv \hat{s}_{k}=\left(\mathcal{T}_{2}^{\varepsilon}\right)^{k}\left(\hat{s}_{0}\right)$ where $\hat{s}_{0}=\tilde{s}+\varepsilon^{\omega+2}$, as long as $d_{\text {min }}^{\varepsilon,+}\left(\hat{s}_{k}\right)$ remains sufficiently small with respect to $R$ (say e.g. $d_{\min }^{\varepsilon,+}\left(\hat{s}_{k}\right) \leq \alpha_{*}^{-\frac{1}{2}} R$ provided $\alpha_{*}$ is chosen sufficiently large), the details are completely similar to the ones in Case II of Proposition 7.4 
and are therefore not repeated here. If we denote by $k_{0}$ the first index for which $d_{\min }^{\varepsilon,+}\left(\hat{s}_{k_{0}}\right)$ becomes larger than $\frac{2}{\mathcal{S}_{2}} r$ (in view of (7.27)) and $k_{1}$ the last index before $d_{\min }^{\varepsilon,+}$ reaches $\alpha_{*}^{-\frac{1}{2}} R$, then we have

$$
\hat{s}_{k_{0}} \leq C r^{\omega+2} \quad \text { and } \quad \hat{s}_{k_{1}} \geq \frac{1}{C} \alpha_{*}^{-(\omega+2) / 2} R^{\omega+2} \geq \frac{1}{C} \alpha_{*}^{(\omega+2) / 2} r^{\omega+2},
$$

for some constant $C>0$ depending only on $M_{0}$ and $V$, and the conclusion that $\mathcal{W} \mathcal{P}_{\varepsilon}^{\mathrm{L}}\left(\alpha_{1} \varepsilon, s_{r}\right)$ holds follows as in case i) above, choosing first $\rho_{*}$ sufficiently large (independently of $\alpha_{*}$ ) and then $\alpha_{*}$ sufficiently large (given $\rho_{*}$ ).

\section{Proofs of Theorem 2, 3 and 4}

\subsection{Proof of Theorem 2}

Theorem 2 being essentially a special case of Theorem 3, we go directly to the proof of Theorem 3. Notice however that in Theorem 2 the solution to the limiting system is unique, so that the result is not constrained by the need to pass to a subsequence.

\subsection{Proof of Theorem 3}

We fix $S<S_{\max }$ and let $\mathrm{L} \geq \kappa_{*}^{-1} \mathrm{~L}_{0}$, where $\mathrm{L}_{0}$ is defined in the statement of Proposition 6 and $\kappa_{*}$ in the statement of Proposition 7. We set $R=\frac{1}{2} \min \left\{a_{k+1}^{0}-a_{k}^{0}, k=1, \cdots, \ell_{0}-1\right\}$ and consider an arbitrary $0<r<R / \alpha_{*}$. Since $\left(\mathrm{H}_{1}\right)$ holds, there exists some constant $\varepsilon_{r}>0$ such that, if $0<\varepsilon \leq \varepsilon_{r}$, then (53) holds with $b_{k} \equiv a_{k}^{0}$ for any $k \in\left\{1, \cdots, \ell_{0}\right\}$. We are therefore in position to make use of Proposition 7 and assert that for all such $\varepsilon$ condition $\mathcal{W P}_{\varepsilon}^{\mathrm{L}}\left(\alpha_{1} \varepsilon, s_{r}\right)$ holds as well as (55) and (56). It follows in particular from (55) and (56) that for every $k \in 1, \cdots, \ell_{0}$ we have $\sharp J_{k}\left(s_{r}\right)=\left|m_{k}^{0}\right|$, where $m_{k}^{0}$ is defined in (12), and therefore $\sharp J\left(s_{r}\right)=\sum_{k=1}^{\ell_{0}}\left|m_{k}^{0}\right| \equiv \ell_{1}$, in other words the number of fronts as well as their properties do not depend on $\varepsilon$ nor on $r$.

We construct next the limiting splitting solution to the ordinary differential equation and the corresponding subsequence proceeding backwards in time and using a diagonal argument. For that purpose, we introduce an arbitrary decreasing sequence $\left\{r_{m}\right\}_{m \in \mathbb{N}_{*}}$ such that $0<$ $r_{1} \leq R / \alpha_{*}$, and such that $r_{m} \rightarrow 0$ as $m \rightarrow+\infty$. For instance, we may take $r_{m}=\frac{1}{m} R / \alpha_{*}$, and we set $s_{m}=s_{r_{m}}$. Taking first $m=1$, we find a subsequence $\left\{\varepsilon_{n, 1}\right\}_{n \in \mathbb{N}_{*}}$ such that $\varepsilon_{n, 1} \rightarrow 0$ as $n \rightarrow \infty$, and such that all points $\left\{a_{k}^{\varepsilon_{n, 1}}\left(s_{1}\right)\right\}_{k \in J}$ converge to some limits $\left\{a_{k}^{1}\left(s_{1}\right)\right\}_{k \in J}$ as $n \rightarrow+\infty$. It follows from (56), passing to the limit $n \rightarrow+\infty$, that

$$
d_{\min }^{*}\left(s_{1}\right) \geq r_{1} .
$$

We are therefore in position to apply the convergence result of Proposition 6, which yields in particular that

$$
\mathfrak{D}_{\varepsilon_{n, 1}}(s) \cap \mathrm{I}_{4 \mathrm{~L}} \longrightarrow \cup_{k=1}^{\ell_{1}}\left\{a_{k}^{1}(s)\right\} \quad \forall s_{1}<s<S_{\max }^{1},
$$

as $n \rightarrow+\infty$, where $\left\{a_{k}^{1}(\cdot)\right\}_{k \in J}$ is the unique solution of $(\mathcal{S})$ with initial data $\left\{a_{k}^{1}\left(s_{1}\right)\right\}_{k \in J}$ on its maximal time of existence $\left(s_{1}, S_{\max }^{1}\right)$. 
Taking next $m=2$, we may extract a subsequence $\left\{\varepsilon_{n, 2}\right\}_{n \in \mathbb{N}_{*}}$ from the sequence $\left\{\varepsilon_{n, 1}\right\}_{n \in \mathbb{N}_{*}}$ such that all the points $\left\{a_{k}^{\varepsilon_{n, 2}}\left(s_{2}\right)\right\}_{k \in J}$ converge to some limits $\left\{a_{k}^{2}\left(s_{2}\right)\right\}_{k \in J}$ as $n \rightarrow+\infty$. Arguing as above, we may assert that

$$
\mathfrak{D}_{\varepsilon_{n, 2}}(s) \cap \mathrm{I}_{4 \mathrm{~L}} \longrightarrow \cup_{k=1}^{\ell_{1}}\left\{a_{k}^{2}(s)\right\} \quad \forall s_{2}<s<S_{\text {max }}^{2},
$$

as $n \rightarrow+\infty$, where $\left\{a_{k}^{2}(\cdot)\right\}_{k \in J}$ is the unique solution of $(\mathcal{S})$ with initial data $\left\{a_{k}^{2}\left(s_{2}\right)\right\}_{k \in J}$ on its maximal time of existence $\left(s_{2}, S_{\max }^{2}\right)$. It follows from (55), namely that only repulsive forces are present at scale smaller than $R$, that $S_{\max }^{2} \geq s_{1}$. Therefore, since we have extracted a subsequence, it follows from (8.2) and (8.3) that $a_{k}^{2}\left(s_{1}\right)=a_{k}^{1}\left(s_{1}\right)$ for all $k \in J$, and therefore also that $S_{\max }^{2}=S_{\max }^{1} \equiv S_{\max }$ and $a_{k}^{2}(\cdot)=a_{k}^{1}(\cdot)=a_{k}(\cdot)$ on $\left(s_{2}, S_{\max }\right)$.

We proceed similarly at each step $m \in \mathbb{N}_{*}$, extracting a subsequence $\left\{\varepsilon_{n, m}\right\}_{n \in \mathbb{N}_{*}}$ from the sequence $\left\{\varepsilon_{n, m-1}\right\}_{n \in \mathbb{N}^{*}}$ such that all the points $\left\{a_{k}^{\varepsilon_{n, m}}\left(s_{m}\right)\right\}_{k \in J}$. Finally setting, for $n \in N_{*}$, $\varepsilon_{n}=\varepsilon_{n, n}$, we obtain that

$$
\mathfrak{D}_{\varepsilon_{n}}(s) \cap \mathrm{I}_{4 \mathrm{~L}} \longrightarrow \cup_{k=1}^{\ell_{1}}\left\{a_{k}(s)\right\} \quad \forall 0<s<S_{\max }^{m},
$$

where $\left\{a_{k}(\cdot)\right\}_{k \in J}$ is a splitting solution of $(\mathcal{S})$ with initial data $\left\{a_{k}^{0}\right\}_{k \in J_{0}}$, on its maximal time of existence $\left(0, S_{\max }\right)$. Since $\mathrm{L} \geq \mathrm{L}_{0}$ was arbitrary, it follows that (15) holds.

It remains to prove that (14). This is actually a direct consequence of (15), the continuity of the trajectories $a_{k}(\cdot)$ and regularizing effect off the front set stated in Proposition 2 (e.g. (31) for the $L^{\infty}$ norm). As a matter of fact, it is standard to deduce from this the fact that the convergence towards the equilibria $\sigma_{q}$, locally outside the trajectory set, holds in any $\mathcal{C}^{m}$ norm, since the potential $V$ was assumed to be smooth.

\subsection{Proof of Theorem 4}

As underlined in the introduction, Theorem 4 follows rather directly from Theorem 3 and more importantly its consequence Corollary 1 (whose proof is elementary and explained after Proposition 1), combined with Theorem 1 and Proposition 2.

Let thus $\mathrm{L}>\mathrm{L}_{0}$ and $\delta>0$ be arbitrarily given, we shall prove that, at least for $\varepsilon \equiv \varepsilon_{n}$ sufficiently small,

$$
\mathcal{D}_{\varepsilon}\left(S_{\max }\right) \cap \mathrm{I}_{\mathrm{L}} \subset \cup_{j \in\left\{1, \cdots, \ell_{2}\right\}}\left[b_{j}-\delta, b_{j}+\delta\right]
$$

and

$$
\left|\mathfrak{v}_{\varepsilon}\left(x, S_{\max }\right)-\sigma_{\hat{\imath}\left(j+\frac{1}{2}\right)}\right| \leq C(\delta, \mathrm{L}) \varepsilon^{\frac{1}{\theta-1}},
$$

for all $j \in\left\{0, \cdots, \ell_{2}\right\}$ and for all $x \in\left(b_{j}+\delta, b_{j+1}-\delta\right)$, where we have used the convention that $b_{0}=-\mathrm{L}$ and $b_{\ell_{2}+1}=\mathrm{L}$. Since $\mathrm{L}$ can be arbitrarily big and $\delta$ arbitrarily small, this will imply that assumption $\left(\mathrm{H}_{1}\right)$ is verified at times $S_{\max }$, which is the claim of Theorem 4 .

Concerning (8.4), by Corollary 1 there exists

$$
s^{-} \in\left[S_{\max }-\rho_{0}\left(\frac{\delta}{4}\right)^{\omega+2}, S_{\max }\right) .
$$

such that

$$
\cup_{k \in\left\{1, \cdots, \ell_{1}\right\}}\left\{a_{k}\left(s^{-}\right)\right\} \subset \cup_{j \in\left\{1, \cdots, \ell_{2}\right\}}\left[b_{j}-\frac{1}{4} \delta, b_{j}+\frac{1}{4} \delta\right] .
$$


The latter and Theorem 3 imply that, for $\varepsilon$ sufficiently small,

$$
\mathcal{D}_{\varepsilon}\left(s^{-}\right) \cap \mathrm{I}_{2 \mathrm{~L}} \cap\left(\cup_{j \in\left\{1, \cdots, \ell_{2}\right\}}\left[b_{j}-\frac{1}{2} \delta, b_{j}+\frac{1}{2} \delta\right]\right)^{c}=\emptyset .
$$

In turn, Theorem 1 (inclusion (7)) and (8.7), combined with the upper bound (8.6) on $S_{\max }-$ $s^{-}$, imply that

$$
\mathcal{D}_{\varepsilon}(s) \cap \mathrm{I}_{\frac{3}{2} \mathrm{~L}} \cap\left(\cup_{j \in\left\{1, \cdots, \ell_{2}\right\}}\left[b_{j}-\frac{3}{4} \delta, b_{j}+\frac{3}{4} \delta\right]\right)^{c}=\emptyset, \quad \forall s \in\left[s^{-}, S_{\max }\right] .
$$

For $s=S_{\max }$ this is stronger than (8.4).

We proceed to (8.5). In view of $(8.8)$, for any $x_{0} \in \mathrm{I}_{\mathrm{L}} \backslash\left(\cup_{j \in\left\{1, \cdots, \ell_{2}\right\}}\left[b_{j}-\delta, b_{j}+\delta\right]\right)$ we have, for $\varepsilon$ sufficiently small,

$$
\mathfrak{v}_{\varepsilon}(y, s) \in B\left(\sigma_{i}, \mu_{0}\right) \quad \forall(y, s) \in\left[x_{0}-\frac{1}{8} \delta, x_{0}+\frac{1}{8} \delta\right] \times\left[s^{-}, S_{\max }\right] .
$$

The latter is nothing but (29) for $r=\frac{1}{8} \delta, s_{0}=s^{-}$and $S=S_{\max }$, and therefore the conclusion (8.5) follows from (31) of Proposition 2, with $C(\delta, \mathrm{L})=\frac{1}{5} \mathrm{C}_{\mathrm{e}}(8 / \delta)^{\frac{1}{\theta-1}}$ as soon as $\varepsilon^{\omega} /\left(S_{\max }-\right.$ $\left.s^{-}\right) \leq \delta^{2} / 64$.

\section{Appendix A}

In this Appendix we establish properties concerning the stationary solutions $\mathfrak{u}^{+}, \stackrel{\vec{u}}{\mathfrak{v}}, \mathfrak{u}_{\varepsilon, r}^{+}$, etc, which we have used in the course of the previous discussion, mainly in Section 5.

\section{A.1 The operator $\mathcal{L}_{\mu}$}

Consider for $\mu>0$ the nonlinear operator $\mathcal{L}_{\mu}$, defined for a smooth functions $U$ on $\mathbb{R}$ by

$$
\mathcal{L}_{\mu}(U)=-\frac{d^{2}}{d x^{2}} U+2 \mu \theta U^{2 \theta-1}
$$

and set for simplicity $\mathcal{L} \equiv \mathcal{L}_{1}$. Most results in this section are deduced from the comparison principle: if $u_{1}$ and $u_{2}$ are two functions defined on some non empty interval $I$, such that

$$
\mathcal{L}_{\mu}\left(u_{1}\right) \geq 0, \mathcal{L}_{\mu}\left(u_{2}\right) \leq 0, \text { and } u_{1} \geq u_{2} \text { on } \partial I,
$$

then $u_{1}(x) \geq u_{2}(x)$ for $x \in I$. Scaling arguments are also used extensively. Given $r>0$ and $\eta>0$ we provide a rescaling of a given smooth function $U$ as follows

$$
\left\{\begin{aligned}
U_{\eta, R} & =\eta U\left(\frac{U}{r}\right), \text { and we verify that } \\
\mathcal{L}_{\mu}\left(U_{\eta, r}\right)(x) & =\frac{\eta}{r^{2}} \mathcal{L}_{\gamma}(U)\left(\frac{x}{r}\right) \text { where } \gamma=\mu \eta^{2(\theta-1)} r^{2} .
\end{aligned}\right.
$$

In particular, if $\mathcal{L}_{\mu}(U)=0$, then we have

$$
\mathcal{L}_{\mu}\left(r^{-\frac{1}{(\theta-1)}} U\left(\frac{\dot{r}}{r}\right)\right)=0 \text { and } \mathcal{L}_{\lambda \mu}\left(\lambda^{-\frac{1}{2(\theta-1)}} U\right)=0 \text {, for any } r>0 \text { and any } \lambda>0 .
$$

Notice also that $U^{*}$ defined on $(0,+\infty)$ by $U^{*}(x)=[\sqrt{2}(\theta-1) x]^{-\frac{1}{\theta-1}}$ solves $\mathcal{L}\left(U^{*}\right)=0$. 
Lemma A.1. There exists a unique smooth map $\underset{\mathfrak{u}_{r}^{+}}{\mathfrak{v}^{+}}$on $(-r, r)$ such that $\mathcal{L}\left(\mathfrak{u}_{r}^{+}\right)=0$ and $\mathfrak{\mathfrak { u }}_{r}^{+}( \pm r)=+\infty$, and a unique solution $\stackrel{\triangleright}{\mathfrak{u}}_{r}$ such that $\mathcal{L}\left(\stackrel{\triangleright}{\mathfrak{u}}_{r}\right)=0$ and $\stackrel{\triangleright}{\mathfrak{u}}_{r}( \pm r)= \pm \infty$. Moreover, $\underset{\mathfrak{u}_{r}^{+}}{\mathfrak{V}^{+}}$is even, $\stackrel{\triangleright}{\mathfrak{u}}_{r}$ is odd, and, setting $\mathfrak{u}^{+} \equiv \mathfrak{u}_{1}^{+}$and $\stackrel{\triangleright}{\mathfrak{u}} \equiv \stackrel{\triangleright}{\mathfrak{u}}_{1}$, we have

$$
\stackrel{\mathfrak{u}}{r}_{r}^{+}(x)=r^{-\frac{1}{\theta-1}} \mathfrak{u}^{+}\left(\frac{x}{r}\right) \text { and } \stackrel{\triangleright}{\mathfrak{u}}_{r}(x)=r^{-\frac{1}{\theta-1}} \stackrel{\triangleright}{\mathfrak{u}}\left(\frac{x}{r}\right) .
$$

Proof. For $n \in \mathbb{N}^{*}$, we construct on $(-r, r)$ a unique solution $\mathfrak{\mathfrak { u }}_{r, n}^{+}$that solves $\mathcal{L}\left(\mathfrak{u}_{r, n}^{+}\right)=0$ and $\mathfrak{\mathfrak { u }}_{r, n}^{+}( \pm r)=n$, minimizing the corresponding convex energy. By the comparison principle, $\mathfrak{\mathfrak { u }}_{r, n}^{+}$is non negative, increasing with $n$ and uniformly bounded on compact subsets of $(-r, r)$ in view of (A.5) below. Hence a unique limit $\mathfrak{u}_{r}^{+}$exists, solution to $\mathcal{L}\left(\mathfrak{u}_{r}^{+}\right)=0$. We observe that $\mathfrak{\mathfrak { u }}_{r, n}^{+}(\cdot) \geq U^{*}\left(r_{n}-\cdot\right)$, where $r_{n}=r+[\sqrt{2}(\theta-1)]^{-1} n^{-(\theta-1)}$, so that we obtain the required boundary conditions for $\mathfrak{\mathfrak { u }}_{r}^{+}$. Uniqueness may again be established thanks to the comparison principle. We construct similarly a unique solution $\stackrel{\triangleright}{\mathfrak{u}}_{r, n}$ that solves $\mathcal{L}\left(\stackrel{\mathrm{u}}{r, n}_{r}\right)=0$ and $\mathfrak{\mathfrak { u }}_{r, n}^{+}( \pm r)= \pm n$. We notice that $\stackrel{\mathrm{u}}{r, n}_{\text {is }}$ is odd, its restriction on $(0, r)$ non negative and increasing with $n$. Moreover, on some interval $(a, r)$, where $0<a<r$ does not depend on $n$, we have $\mathfrak{\mathfrak { u }}_{r, n}^{+}(\cdot) \geq U^{*}\left(\tilde{r}_{n}-\cdot\right)$ where $\tilde{r}_{n}=r+[(\theta-1)]^{-1} n^{-(\theta-1)}$, and we conclude as for the first assertion.

Remark A.1. Given $r>0$ and $\lambda>0$ we notice that the function $\stackrel{\vee}{\mathrm{U}_{r}^{\lambda}}$ and $\stackrel{\triangleright}{\mathrm{U}}{ }_{r}^{\lambda}$ defined by

$$
\stackrel{\vee}{\mathrm{U}_{r}^{\lambda}}(x)=\lambda^{-\frac{1}{2(\theta-1)}} \mathfrak{u}_{r}^{+}(x) \text { and } \stackrel{\triangleright}{\mathrm{U}_{r}^{\lambda}}(x)=\lambda^{-\frac{1}{2(\theta-1)}} \stackrel{\mathfrak{u}}{r}_{r}(x)
$$

solve $\stackrel{\vee}{\mathcal{L}_{\lambda}\left(\mathrm{U}_{r}^{\lambda}\right)}=0$ and $\left.\stackrel{\triangleright}{\mathcal{L}_{\lambda}\left(\mathrm{U}_{r}^{\lambda}\right.}\right)=0$ with the same boundary conditions as $\stackrel{\mathfrak{u}_{r}^{+}}{\mathfrak{u}_{r}}$ and $\stackrel{\mathfrak{u}_{r}}{\text {. }}$

Lemma A.2. i) Assume that $\mathcal{L}(u) \leq 0$ on $(-r, r)$. Then, we have, for $x \in(-r, r)$

$$
u(x) \leq(\sqrt{2}(\theta-1))^{-\frac{1}{\theta-1}}\left[(x+r)^{-\frac{1}{\theta-1}}+(x-r)^{-\frac{1}{\theta-1}}\right] .
$$

ii) Assume that $\mathcal{L}(u) \geq 0$ on $(-r, r)$ and that $u(-r)=u(r)=+\infty$. Then we have

$$
u(x) \geq(\sqrt{2}(\theta-1))^{-\frac{1}{\theta-1}} \max \left\{(x+r)^{-\frac{1}{\theta-1}},(r-x)^{-\frac{1}{\theta-1}}\right\} .
$$

Proof. Set $\tilde{U}=U^{*}(\cdot+r)+U^{*}(r-\cdot)$. By subaddivity and translation invariance, we have $\mathcal{L}(\tilde{U}) \geq 0$ on $(-r, r)$ with $\tilde{U}( \pm r)=+\infty$, so that (A.5) follows from the comparison principle (A.1) with $u_{1}=\tilde{U}$ and $u_{2}=u$. Similarly, (A.6) follows from (A.1) with $u_{1}=u$ and $u_{2}=U^{*}(\cdot+r)$ or $u_{2}=U^{*}(r-\cdot)$.

Combining estimate ii) of Lemma A.2 with the scaling law of Lemma A.1 we are led to

$$
\left|\frac{d}{d r} \mathfrak{u}_{r}^{+}(x)\right|+\left|\frac{d}{d r} \mathfrak{u}_{r}(x)\right| \leq C r^{-\frac{\theta}{\theta-1}}, \text { for } x \in\left(-\frac{7}{8} r, \frac{7}{8} r\right) .
$$




\section{A.2 The discrepancy for $\mathcal{L}_{\mu}$}

The discrepancy $\Xi_{\mu}$ for $\mathcal{L}_{\mu}$ relates to a given function $u$ the function $\Xi_{\mu}(u)$ defined by

$$
\Xi_{\mu}(u)=\frac{\dot{u}^{2}}{2}-\mu u^{2 \theta}
$$

This function is constant if $u$ solves $\mathcal{L}_{\mu}(u)=0$. We set $\Xi=\Xi_{1}$,

$$
A_{\theta} \equiv \Xi\left(\mathfrak{u}^{+}\right)=-\left(\mathfrak{u}^{+}(0)\right)^{2 \theta}<0 \text { and } B_{\theta} \equiv \Xi(\stackrel{\triangleright}{\mathfrak{u}})=\frac{(\stackrel{\triangleright}{\mathfrak{u}}(0))_{x}^{2}}{2}>0 .
$$

In view of the scaling relations (A.3) and Remark A.1, we are hence led to the identities

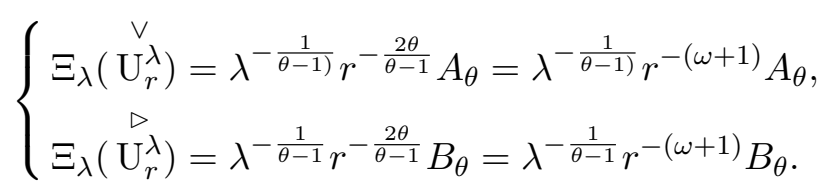

\section{A.3 The operator $\mathcal{L}^{\varepsilon}$}

In this subsection, we consider more generally, for given $\lambda>0$ the operator $\mathcal{L}^{\varepsilon}$ given by

$$
\mathcal{L}^{\varepsilon}(U)=-\frac{d^{2}}{d x^{2}} U+2 \lambda f_{\varepsilon}(U)
$$

with $f_{\varepsilon}$ defined in (5.4), and the solutions $\underset{\mathfrak{u}_{\varepsilon, r}^{+}}{\mathfrak{v}^{+}}, \mathfrak{\mathfrak { u }}_{\varepsilon, r}^{-}$, and $\stackrel{\triangleright}{\mathfrak{u}, r}_{\text {of }} \mathcal{L}^{\varepsilon}(U)=0$ on $(-r, r)$ with corresponding infinite boundary conditions, whose existence and uniqueness is proved as for Lemma A.1.

Lemma A.3. We have the estimates

$$
\left|\mathfrak{u}_{\varepsilon, r}^{+}(x)\right|+\left|\stackrel{\triangleright}{\mathfrak{u}}_{\varepsilon, r}(x)\right| \leq C\left(\lambda^{2}(\theta-1)\right)^{-\frac{1}{\theta-1}}\left[(x+r)^{-\frac{1}{\theta-1}}+(x-r)^{-\frac{1}{\theta-1}}\right] .
$$

Proof. It follows from (5.5) that $\mathcal{L}_{\frac{3}{4} \lambda}\left(\mathfrak{u}_{\varepsilon, r}+\underset{ }{v_{1}}\right) \leq 0$, so that, invoking the comparison principle as well as the scaling law (A.2) we deduce that $\mathfrak{\mathfrak { u }}_{\varepsilon, r}^{+} \leq(3 \lambda / 4)^{-2(\theta-1)}{\mathfrak{\mathfrak { u } ^ { + }}}_{r}^{+}$. A similar estimate holds for $\stackrel{\triangleright}{\mathfrak{u}, r}_{\varepsilon}$ and the conclusion follows from Lemma A.2.

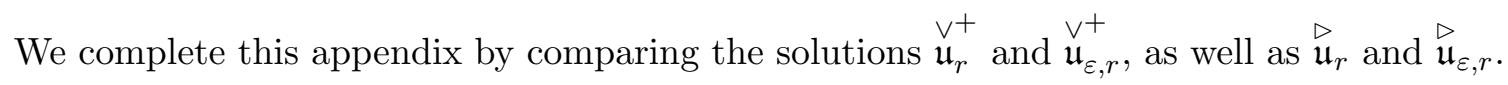

Proposition A.1. In the interval $\left(-\frac{7}{8} r, \frac{7}{8} r\right)$ we have the estimate

$$
\left|\mathfrak{u}_{\varepsilon, r}^{+}-\mathfrak{u}_{r}^{+}\right| \leq C \varepsilon^{\frac{1}{\theta}} r^{-\frac{2 \theta-1}{\theta(\theta-1)}} .
$$

Proof. Let $\varepsilon<\delta<r / 16$ to be fixed. It follows from Lemma A.3 that, for $x \in(-r+\delta, r-\delta)$, we have

$$
0 \leq \varepsilon^{\frac{1}{\theta-1}} \mathfrak{u}_{\varepsilon, r}^{+}(x) \leq C\left(\frac{\varepsilon}{\delta}\right)^{\frac{1}{\theta-1}},
$$


and therefore also

$$
\left|\varepsilon^{\frac{1}{\theta-1} \mathfrak{u}_{\varepsilon, r}^{+}}(x) g\left(\varepsilon^{\frac{1}{\theta-1} \mathfrak{u}_{\varepsilon, r}^{+}}(x)\right)\right| \leq C\left(\frac{\varepsilon}{\delta}\right)^{\frac{1}{\theta-1}}
$$

It follows from (A.11) and the fact that $\mathcal{L}^{\varepsilon}\left(\mathfrak{u}_{\varepsilon, r}^{+}\right)=0$, that $\mathcal{L}_{\lambda_{\varepsilon}^{-}}\left(\mathfrak{u}_{\varepsilon, r}^{+}\right) \leq 0$, where $\lambda_{\varepsilon}^{ \pm}=$ $\lambda\left(1 \pm C\left(\frac{\varepsilon}{\delta}\right)^{\frac{1}{\theta-1}}\right)$. On the other hand, by the scaling law (A.2), we have

$$
\mathcal{L}_{\lambda_{\varepsilon}^{-}}\left(\left(\frac{\lambda_{\varepsilon}^{-}}{\lambda}\right)^{-\frac{1}{2(\theta-1)}} \stackrel{\mathfrak{u}}{+}+\delta_{r-\delta}^{+}=0\right.
$$

It follows from the comparison principle, since the second function is infinite on the boundary of the interval $[-r+\delta, r-\delta]$, that

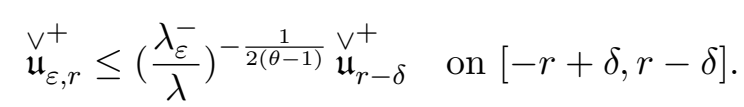

Integrating the inequality (A.7) between $r-\delta$ and $r$, we deduce that for $x \in\left(-\frac{7}{8} r, \frac{7}{8} r\right)$, we have the inequality

$$
\left|\mathfrak{u}_{r-\delta}^{+}(x)-\mathfrak{u}_{r}^{+}(x)\right| \leq C \delta r^{-\frac{\theta}{\theta-1}} .
$$

On the other hand, it follows from estimate (A.5) of Lemma A.2 that for $x \in\left(-\frac{7}{8} r, \frac{7}{8} r\right)$,

$$
\left|\left(\frac{\lambda_{\varepsilon}^{-}}{\lambda}\right)^{-\frac{1}{2(\theta-1)}} \underset{\mathfrak{u}_{r-\delta}^{+}}{\mathfrak{v}_{r}}(x)-\mathfrak{\mathfrak { u }}_{r-\delta}^{+}(x)\right| \leq C\left(\frac{\varepsilon}{\delta}\right)^{\frac{1}{\theta-1}} r^{-\frac{1}{\theta-1}} .
$$

We optimize the outcome of (A.12) and (A.13) choosing $\delta:=\varepsilon^{\frac{1}{\theta}} r^{\frac{\theta-1}{\theta}}$ and we therefore obtain

$$
\mathfrak{\mathfrak { u }}_{\varepsilon, r}^{+} \leq \mathfrak{u}_{r}^{+}+C \varepsilon^{\frac{1}{\theta}} r^{-\frac{2 \theta-1}{\theta(\theta-1)}} \quad \text { on }\left(-\frac{7}{8} r, \frac{7}{8} r\right)
$$

The lower bound for $\mathfrak{u}_{\varepsilon, r}^{+}$is obtained in a similar way but reversing the role of super and subsolutions: the function $\left(\frac{\lambda_{\varepsilon}^{+}}{\lambda}\right)^{-\frac{1}{2(\theta-1)}} \mathfrak{u}_{r+\delta}^{+}$yields a subsolution for $\mathcal{L}^{\varepsilon}$ on $[-r, r]$ whereas $\mathfrak{u}_{\varepsilon, r}^{+}$ is a solution. The conclusion then follows.

Similarly, we have:

Proposition A.2. In the interval $\left(-\frac{7}{8} r, \frac{7}{8} r\right)$ we have the estimate

$$
\left|\overrightarrow{\mathfrak{u}}_{\varepsilon, r}-\overrightarrow{\mathfrak{u}}_{r}\right| \leq C \varepsilon^{\frac{1}{\theta}} r^{-\frac{2 \theta-1}{\theta(\theta-1)}} .
$$

Proof. We only sketch the necessary adaptations since the argument is closely parallel to the proof of Proposition A.1. First, by the maximum principle $\mathfrak{\mathfrak { u }}_{\varepsilon, r}$ can only have negative maximae and positive minimae, so that actually $\mathfrak{u}_{\varepsilon, r}$ has no critical point and a single zero, which we call $a_{\varepsilon}$. Arguing as in Proposition A.1, one first obtains

$$
\left(\lambda_{\varepsilon}^{-} / \lambda\right)^{-\frac{1}{2(\theta-1)}} \stackrel{\triangleright}{\mathfrak{u}}_{r-\delta-a_{\varepsilon}}\left(\cdot-a_{\varepsilon}\right) \geq{\stackrel{\triangleright}{\mathfrak{u}_{\varepsilon, r}}} \geq\left(\lambda_{\varepsilon}^{+} / \lambda\right)^{-\frac{1}{2(\theta-1)}} \stackrel{\triangleright}{\mathfrak{u}}_{r+\delta-a_{\varepsilon}}\left(\cdot-a_{\varepsilon}\right) \quad \text { on }\left[a_{\varepsilon}, r-\delta\right],
$$

and

$-\left(\lambda_{\varepsilon}^{-} / \lambda\right)^{-\frac{1}{2(\theta-1)}}{\stackrel{\vec{u}}{r+a_{\varepsilon}-\delta}}\left(\cdot-a_{\varepsilon}\right) \geq-\overrightarrow{\mathfrak{u}}_{\varepsilon, r} \geq-\left(\lambda_{\varepsilon}^{+} / \lambda\right)^{-\frac{1}{2(\theta-1)}}{\stackrel{\vec{u}}{r+a_{\varepsilon}+\delta}}\left(\cdot-a_{\varepsilon}\right) \quad$ on $\left[-r+\delta, a_{\varepsilon}\right]$. 
Since $\stackrel{\triangleright}{\mathfrak{u}, r}_{\text {r }}$ is continuously differentiable at the point $a_{\varepsilon}$ (indeed it solves $\mathcal{L}^{\varepsilon}(\stackrel{\mathrm{u}}{\varepsilon, r})=0$ ), and since the derivative at zero of the function $\stackrel{b}{u}_{r}$ is a deacreasing function of $r$, it first follows from the last two sets of inequalities that $\left|a_{\varepsilon}\right| \leq \delta$, and the conclusion then follows as in Proposition A.1.

\section{Appendix B}

\section{B.1 Some properties of the ordinary differential equation $(\mathcal{S})$}

This Appendix is devoted to properties of the system of ordinary differential equations $(\mathcal{S})$, the result being somewhat parallel to the results in Section 2 of [5]. We assume that we are given $\ell \in \mathbb{N}^{*}$, and a solution, for $k \in J=\{1, \cdots, \ell\} t \mapsto a(t)=\left(a_{1}(t), \cdots, a_{\ell}(t)\right)$ to the system

$$
\mathfrak{q}_{k} \frac{d}{d s} a_{k}(s)=-\mathcal{B}_{\left(k-\frac{1}{2}\right)}\left[\left(a_{k}(s)-a_{k-1}(s)\right]^{-(\omega+1)}+\mathcal{B}_{\left(k+\frac{1}{2}\right)}\left[\left(a_{k+1}(s)-a_{k}(s)\right]^{-(\omega+1)},\right.\right.
$$

where the numbers $\mathfrak{q}_{k}$ are supposed to be positive, and are actually taken in $(\mathcal{S})$ equal to $\mathfrak{S}_{i(k)}$, whereas the numbers $\mathcal{B}_{k+\frac{1}{2}}$, which may have positive or negative signs, are taken in $(\mathcal{S})$ to be equal to $\Gamma_{i\left(k-\frac{1}{2}\right)}$. We also define $\mathfrak{q}_{\min }=\min \left\{\mathfrak{q}_{i}\right\}$ and $\mathfrak{q}_{\max }=\max \left\{\mathfrak{q}_{i}\right\}$. We consider a solution on its maximal interval of existence, which we call $\left[0, T_{\max }\right]$. An important feature of the equation (B.1) is its gradient flow structure. The behavior of this system is indeed related to the function $F$ defined on $\mathbb{R}^{\ell}$ by

$$
\left\{\begin{aligned}
F(U) & =\sum_{k=0}^{\ell-1} F_{k+\frac{1}{2}}(U), \text { where, for } k=0, \cdots, \ell-1, \text { and } U=\left(u_{1}, \cdots, u_{\ell}\right), \text { we set } \\
F_{k+\frac{1}{2}}(U) & =-\omega^{-1} \mathcal{B}_{k+\frac{1}{2}}\left(u_{k+1}-u_{k}\right)^{-\omega} \text { with the convention that } u_{0}=-\infty .
\end{aligned}\right.
$$

If $u_{1}<u_{2}<\cdots<u_{\ell}$, for $k=1, \ldots, \ell$, then we have

$$
\frac{\partial F}{\partial u_{k}}(U)=\mathcal{B}_{k-\frac{1}{2}}\left(u_{k}-u_{k-1}\right)^{-(\omega+1)}-\mathcal{B}_{k+\frac{1}{2}}\left(u_{k+1}-u_{k}\right)^{-(\omega+1)},
$$

so that $(\mathcal{S})$ writes $\frac{d}{d s} a_{k}(s)=-\mathfrak{q}_{k}^{-1} \frac{\partial F}{\partial u_{k}}(a(s))$. Hence, we have

$$
\frac{d}{d t} F(a(t))=\sum_{k=1}^{\ell} \frac{\partial F}{\partial u_{k}}(a(t)) \frac{d a_{k}}{d t}(t)=-\sum_{k=1}^{\ell} \mathfrak{q}_{k}^{-1}\left(\frac{\partial F}{\partial u_{k}}(a(t))\right)^{2} \leq-\mathfrak{q}_{\max }^{-1}|\nabla F(a(t))|^{2},
$$

hence $F$ decreases along the flow. We also consider the positive functionals defined by

$$
F_{\text {rep }}(U)=\sum_{k \in J^{+}} F_{k+\frac{1}{2}}(U), F_{\text {att }}=-\sum_{k \in J^{-}} F_{k+\frac{1}{2}}(U), \text { for } U=\left(u_{1}, \cdots, u_{\ell}\right),
$$

where $J^{ \pm}=\left\{k \in\{0, \ell-1\}\right.$ such that $\left.\epsilon_{k+\frac{1}{2}} \equiv \operatorname{sgn}\left(\mathcal{B}_{k+\frac{1}{2}}\right)= \pm 1\right\}$. 
Proposition B.1. Let $a=\left(a_{1}, \cdots, a_{\ell}\right)$ be a solution to (B.1) on its maximal interval of existence $\left[0, T_{\max }\right]$. Then, we have, for any time $t \in\left[0, T_{\max }\right]$

$$
\left\{\begin{array}{l}
\left(F_{\text {rep }}(a(t))\right)^{-\frac{\omega+2}{\omega}} \geq\left(F_{\text {rep }}(a(0))\right)^{-\frac{\omega+2}{\omega}}+\mathcal{S}_{0} t, \quad \boldsymbol{\delta}_{a}^{+}(t) \geq\left(\mathcal{S}_{1} t+\mathcal{S}_{2} \boldsymbol{\delta}_{a}^{+}(0)^{\omega+2}\right)^{\frac{1}{\omega+2}} \\
\left(F_{\text {att }}(a(t))\right)^{-\frac{\omega+2}{\omega}} \leq\left(F_{\text {att }}(a(0))\right)^{-\frac{\omega+2}{\omega}}-\mathcal{S}_{0} t, \quad \boldsymbol{\delta}_{a}^{-}(t) \leq\left(\mathcal{S}_{3} \boldsymbol{\delta}_{a}^{-}(0)^{\omega+2}-\mathcal{S}_{4} t\right)^{\frac{1}{\omega+2}}
\end{array}\right.
$$

where $\mathcal{S}_{0}>0, \mathcal{S}_{1}>0, \mathcal{S}_{2}>0, \mathcal{S}_{3}>0$ and $\mathcal{S}_{4}>0$ depend only the coefficients of (B.1).

Since $\boldsymbol{\delta}_{a}^{-}(s) \geq 0$, an immediate consequence of (B.4) is that

$$
T_{\max } \leq \frac{\mathcal{S}_{3}}{\mathcal{S}_{4}} \delta_{a}^{-}(0)
$$

Since the system (B.1) involves both attractive and repulsive forces, for the proof of Proposition B.1 it is convenient to divide the collection $\left\{a_{1}(t), a_{2}(t), \cdots, a_{\ell}(t)\right\}$ into repulsive and attractive chains. We say that a subset $A$ of $J$ is a chain if $A=\{k, k+1, k+2, \cdots, k+m\}$ is an ordered subset of $m$ consecutive elements in $J$, with $m \geq 1$.

Definition 4. A chain $A$ is said to be repulsive (resp. attractive) if and only if $\epsilon_{j+\frac{1}{2}}=-1$ (resp. +1) for $j=k, \cdots, k+m$. It is said to be a maximal repulsive chain (resp. maximal attractive chain), if there does exists any repulsive (resp. attractive) chain which contains $A$ strictly.

It follows from our definition that repulsive or attractive chain contain at least two elements. We may decompose $J$, in increasing order, as

$$
J=B_{0} \cup A_{1} \cup B_{1} \cup A_{2} \cup B_{2} \cup \cdots \cup B_{p-1} \cup A_{p} \cup B_{p},
$$

where the chains $A_{i}$ are maximal repulsive chains, the chains $B_{i}$ are maximal attractive for $i=1, \ldots, p-1$, and the sets $B_{0}$ and $B_{p}$ being possibly empty or maximal attractive chains. Moreover for $i=1 \cdots, p$ the sets $A_{i} \cap B_{i}$, and $B_{i} \cap A_{i+1}$ contain one element.

\section{B.2 Maximal repulsive chains}

In this subsection, we restrict ourselves to the study of the behavior of a maximal repulsive chain $A=\{j, j+1, \ldots j+m\}$ of $m+1$ consecutive points, $m \leq \ell-2$ within the general system (B.1). Setting, for $k=0, \cdots, m, \mathfrak{u}_{k}(\cdot)=a_{k+j}(\cdot)$, we are led to study $\mathfrak{u}(\cdot)=\left(\mathfrak{u}_{0}(\cdot), \mathfrak{u}_{1}(\cdot), \cdots, \mathfrak{u}_{m}(\cdot)\right)$. Since $B_{k+\frac{1}{2}}<0$ in the repulsive case the chain $\mathfrak{u}$ is moved through a system of $m-1$ ode's,

$$
\mathfrak{q}_{k} \frac{d}{d s} \mathfrak{u}_{k}(s)=-\left|\mathcal{B}_{\left(k-\frac{1}{2}\right)}\right|\left[\left(\mathfrak{u}_{k}(s)-\mathfrak{u}_{k-1}(s)\right]^{-(\omega+1)}+\left|\mathcal{B}_{\left(k+\frac{1}{2}\right)}\right|\left[\left(\mathfrak{u}_{k+1}(s)-\mathfrak{u}_{k}(s)\right]^{-(\omega+1)}\right.\right.
$$

for $k=1, \cdots, m-1$, whereas the end points satisfy two differential inequalities

$$
\frac{d}{d s} \mathfrak{u}_{m}(s) \geq-\mathfrak{q}_{m}^{-1} \frac{\partial \tilde{F}_{\text {rep }}}{\partial u_{m}}\left(\mathfrak{u}_{m}(s)\right), \quad \frac{d}{d s} \mathfrak{u}_{0}(s) \leq-\mathfrak{q}_{0}^{-1} \frac{\partial \tilde{F}_{\text {rep }}}{\partial u_{0}}(a(s)),
$$

where we have set $\tilde{F}_{\text {rep }}(U)=\sum_{k=0}^{m-1} F_{k+\frac{1}{2}}(U)$. We assume that at initial time we have

$$
\mathfrak{u}_{0}(0)<\mathfrak{u}_{1}(0)<\cdots<\mathfrak{u}_{m}(0) .
$$

Set $\boldsymbol{\delta}_{\mathfrak{u}}(t)=\min \left\{\mathfrak{u}_{k+1}(t)-\mathfrak{u}_{k}(t), \quad k=0, \cdots, m-1\right\}$. We prove in this subsection: 
Proposition B.2. Assume that the function $\mathfrak{U}$ satisfies the system (B.7) and (B.8) on $\left[0, T_{\max }\right]$, and that (B.9) hold. Then, we have, for any $t \in\left[0, T_{\max }\right]$

$$
\begin{gathered}
\left(\tilde{F}_{\text {rep }}(\mathfrak{H}(t))\right)^{-\frac{\omega+2}{\omega}}-\left(\tilde{F}_{\text {rep }}(\mathfrak{U}(0))\right)^{-\frac{\omega+2}{\omega}} \geq \frac{\omega+1}{4 \omega} \mathfrak{q}_{\max }^{-1}\left(\omega \mathcal{B}_{\max }\right)^{-\frac{2(\omega+1)}{\omega}} t, \text { so that } \\
\boldsymbol{\delta}_{\mathfrak{u}}(t) \geq\left(\mathcal{S}_{1} t+\mathcal{S}_{2} \boldsymbol{\delta}_{\mathfrak{u}}(0)^{\omega+2}\right)^{\frac{1}{\omega+2}},
\end{gathered}
$$

where $\mathcal{S}_{1}>0$ and $\mathcal{S}_{2}>0$ depend only on the coefficients of the equation (B.1).

The proof relies on several elementary observations.

Lemma B.1. Let $\mathfrak{u}$ be a solution to (B.7), (B.8) and (B.9). Then, we have,

$$
\frac{d}{d t} \tilde{F}_{\text {rep }}(\mathfrak{u}(t)) \leq-\mathfrak{q}_{\max }^{-1}\left|\nabla \tilde{F}_{\text {rep }}(\mathfrak{u}(t))\right|^{2}, \text { for every } t \in\left[0, T_{\text {max }}\right] .
$$

The proof is similar to (B.3) and we omit it. For $U=\left(u_{0}, u_{1}, \cdots, u_{m}\right) \in \mathbb{R}^{m+1}$ with $u_{0}<\cdots<u_{m}$ set $\rho_{\min }(U)=\inf \left\{\left|u_{k+1}-u_{k}\right|, k=0, \cdots, m-1\right\}$ and $\mathcal{B}_{\min }=\inf \left\{\left|\mathcal{B}_{k+\frac{1}{2}}\right|\right\}$, $\mathcal{B}_{\max }=\sup \left\{\left|\mathcal{B}_{k+\frac{1}{2}}\right|\right\}$.

Lemma B.2. Let $U=\left(u_{0}, \cdots, u_{m}\right)$ be such that $u_{0}<u_{1}<\cdots<u_{m}$. We have,

$$
\begin{array}{r}
\mathcal{B}_{\min } \omega^{-1} \rho_{\min }(U)^{-\omega} \leq \tilde{F}_{\text {rep }}(U) \leq \mathcal{B}_{\max }(m+1)(\omega)^{-1} \rho_{\min }(U)^{-\omega} . \\
\left|\nabla \tilde{F}_{\text {rep }}(U)\right| \leq(m+2) \mathcal{B}_{\max }\left((\omega-1) \mathcal{B}_{\min }\right)^{-\frac{\omega+1}{\omega}}\left(\tilde{F}_{\text {rep }}(U)\right)^{\frac{\omega+1}{\omega}}, \\
\left|\frac{\partial \tilde{F}_{\text {rep }}(U)}{\partial u_{k}}\right| \geq \frac{1}{2}\left(\omega \mathcal{B}_{\max }\right)^{-\frac{\omega+1}{\omega}}\left(\tilde{F}_{\text {rep }}(U)\right)^{\frac{\omega+1}{\omega}}, \text { for every } k=0, \cdots, m .
\end{array}
$$

Proof. Inequalities (B.13) and (B.14) are direct consequences of the definition of $\tilde{F}_{\text {rep }}$. We turn therefore to estimate (B.15). In view of formula (B.2), the cases $k=0$ and $k=$ $m+1$ are straightforward. Next, let $k=1, \cdots m$ and set $T_{k+\frac{1}{2}}=\mathcal{B}_{k+\frac{1}{2}}\left(u_{k+1}-u_{k}\right)^{-(\omega+1)}$. We distinguish two cases:

Case 1: $T_{k-\frac{1}{2}} \leq \frac{1}{2} T_{k+\frac{1}{2}}$. Then, we have, in view of (B.2) $T_{k+\frac{1}{2}} \leq 2\left|\frac{\partial F}{\partial u_{k}}(U)\right| \leq 2|\nabla F(U)|$, and we are done.

Case 2: $T_{k-\frac{1}{2}} \geq \frac{1}{2} T_{k+\frac{1}{2}}$. In that case, we repeat the argument with $k$ replaced by $k-1$. Then either $T_{k-\frac{3}{2}} \leq \frac{1}{2} T_{k-\frac{1}{2}}$, which yields as above $T_{k-\frac{1}{2}} \leq 2|\nabla F(U)|$, so that we are done, or $T_{k-\frac{3}{2}} \geq \frac{1}{2} T_{k+\frac{1}{2}}$, and we go on. Since we have to stop at $k=0$, this leads to the desired inequality (B.15).

Proof of Proposition B.2. Combining (B.12) with (B.15) we are led to

$$
\frac{d}{d t} \tilde{F}_{\text {rep }}(\mathfrak{u}(t)) \leq-\frac{1}{4} \mathfrak{q}_{\max }^{-1}\left(\omega \mathcal{B}_{\text {max }}\right)^{-\frac{2(\omega+1)}{\omega}}\left(\tilde{F}_{\text {rep }}(\mathfrak{u}(t))\right)^{\frac{2(\omega+1)}{\omega}} .
$$

Integrating this differential equation, we obtain (B.10). Combining the last inequality of Lemma B.1 with inequality (B.13), inequality (B.11) follows. 


\section{B.3 Maximal attractive chains}

Maximal attractive chains $B=\{j, j+1, \ldots j+m\}$, with $m \leq \ell-1$ within the general system (B.1), are handled similarly. Defining $\mathfrak{U}$ as above, the function $\mathfrak{U}$ still satisfies (B.7), but the inequalities (B.8) are now replaced by

$$
\frac{d}{d s} \mathfrak{u}_{m}(s) \leq-\mathfrak{q}_{m}^{-1} \frac{\partial \tilde{F}_{\text {att }}}{\partial u_{m}}\left(\mathfrak{u}_{m}(s)\right), \quad \frac{d}{d s} \mathfrak{u}_{0}(s) \geq-\mathfrak{q}_{0}^{-1} \frac{\partial \tilde{F}_{\text {att }}}{\partial u_{0}}(a(s))
$$

$\tilde{F}_{\text {att }}(U)$ is defined by $\tilde{F}_{\text {att }}=-\tilde{F}_{\text {rep }}$, so that we have in the attractive case $\tilde{F}_{\text {att }} \geq 0$. Up to a change of sign, the function $\tilde{F}_{\text {att }}$ verifies the properties (B.13), (B.14) and (B.15) stated in Proposition B.2. However the differential inequality (B.12) is now turned into

$$
\frac{d}{d t} \tilde{F}_{\text {att }}(\mathfrak{u}(t)) \geq \mathfrak{q}_{\max }^{-1}\left|\nabla \tilde{F}_{\text {att }}(\mathfrak{H}(t))\right|^{2} \geq \mathcal{C} \tilde{F}_{\text {att }}(\mathfrak{u}(t))^{\frac{2(\omega+1)}{\omega}},
$$

where the last inequality follows from (B.15) and where $\mathcal{C}$ is some constant depending only on the coefficients in (B.1). Integrating (B.17) and invoking once more (B.13), we obtain

Lemma B.3. Assume that $\mathfrak{u}$ satisfies the system (B.7) and (B.16) on $\left[0, T_{\max }\right]$ with (B.9). Then for constants $\mathcal{S}_{3}>0$ and $\mathcal{S}_{4}>0$ depending only on the coefficients of (B.1), we have

$$
\boldsymbol{\delta}_{\mathfrak{u}}(t) \leq\left(\mathcal{S}_{3} \boldsymbol{\delta}_{\mathfrak{u}}(0)^{\omega+2}-\mathcal{S}_{4} t\right)^{\frac{1}{\omega+2}} .
$$

\section{B.4 Proof of Proposition B.1 completed}

Inequalities (B.4) of Proposition B.1 follow immediately from Proposition B.2 and Lemma B.3 applied to each separate maximal chain provided by the decomposition (B.6).

\section{References}

[1] F. Bethuel, H. Brezis and F. Hélein Ginzburg-Landau vortices, Birkhaüser (1994).

[2] F. Bethuel, G.Orlandi and D. Smets, Collisions and phase-vortex interactions in dissipative Ginzburg-Landau dynamics, Duke Math. J. 130 (2005) 523-614.

[3] F Bethuel, G.Orlandi, D. Smets, Dynamics of multiple degree Ginzburg-Landau vortices, Comm. Math. Phys. 272 (2007) 229-261.

[4] F. Bethuel, G. Orlandi and D. Smets, Slow motion for gradient systems with equal depth multiple-well potentials, J. Diff. Equations 250 (2011), 53-94.

[5] F. Bethuel, and D. Smets, Slow motion for equal depth multiple-well gradient systems: the degenerate case, Discrete Contin. Dyn. Syst. 33 (2013), 67-87.

[6] F. Bethuel and D. Smets, On the motion law of fronts for scalar reaction-diffusion equations with equal depth multiple-well potentials, preprint.

[7] L. Bronsard and R.V. Kohn, On the slowness of phase boundary motion in one space dimension, Comm. Pure Appl. Math. 43 (1990), 983-997. 
[8] X. Chen, Generation, propagation, and annihilation of metastable patterns. J. Differential Equations 206 (2004), no. 2, 399-437.

[9] J. Carr, and R.L. Pego, Metastable patterns in solutions of $u_{t}=\epsilon^{2} u_{x x}-f(u)$. Comm. Pure Appl. Math. 42 (1989), 523-576.

[10] G. Fusco, J.K. Hale, Slow-motion manifolds, dormant instability, and singular perturbations J. Dynam. Differential Equations 1 (1989), 75-94.

[11] R.L. Jerrard and H.M. Soner, Dynamics of Ginzburg-Landau vortices, Arch. Rational Mech. Anal. 142 (1998), 99-125.

[12] J. B. Keller, On solutions of $\Delta u=f(u)$, Comm. Pure Appl. Math. 10 (1957), 503-510.

[13] F.H. Lin, Some dynamical properties of Ginzburg-Landau vortices, Comm. Pure Appl. Math. 49 (1996), 323-359.

[14] R. Osserman, On the inequality $\Delta u \geq f(u)$, Pacific J. Math. 7 (1957), 1641-1647.

[15] E. Sandier and S. Serfaty, Gamma-convergence of gradient flows with applications to Ginzburg-Landau, Comm. Pure Appl. Math. 57 (2004), 1627-1672. 